\title{
Off-diagonal terms in Yukawa textures of the Type-III 2-Higgs doublet model and light charged Higgs boson phenomenology
}

\section{J. Hernández-Sánchez ${ }^{a}$ S. Moretti ${ }^{b}$ R. Noriega-Papaqui ${ }^{c}$ A. Rosado ${ }^{d}$}

${ }^{a}$ Fac. de Cs. de la Electrónica, Benemérita Universidad Autónoma de Puebla, Apdo. Postal 542, 72570 Puebla, Puebla, México and Dual C-P Institute of High Energy Physics, México.

${ }^{b}$ School of Physics and Astronomy, University of Southampton, Highfield, Southampton SO17 1BJ, United Kingdom, and Particle Physics Department, Rutherford Appleton Laboratory, Chilton, Didcot, Oxon OX11 0QX, United Kingdom

${ }^{c}$ Área Académica de Matemáticas y Física, Universidad Autónoma del Estado de Hidalgo, Carr. Pachuca-Tulancingo Km. 4.5, C.P. 42184, Pachuca, Hgo. and Dual C-P Institute of High Energy Physics, México.

${ }^{d}$ Instituto de Física, BUAP, Apdo. Postal J-48, C.P. 72570 Puebla, Pue., México.

E-mail: jaimeh@ece.buap.mx, s.moretti@soton.ac.uk,

rnoriega@uaeh.edu.mx, rosado@ifuap.buap.mx

ABSTRACT: We discuss flavor-violating constraints and consequently possible charged Higgs boson phenomenology emerging from a four-zero Yukawa texture embedded within the Type-III 2-Higgs Doublet Model (2HDM-III). Firstly, we show in detail how we can obtain several kinds of $2 \mathrm{HDM}$ shen some parameters in the Yukawa texture are absent. Secondly, we present a comprehensive study of the main $B$-physics constraints on such parameters induced by flavor-changing processes, in particular on the off-diagonal terms of such a texture: i.e., from $\mu-e$ universality in $\tau$ decays, several leptonic $B$-decays $(B \rightarrow \tau \nu$, $D \rightarrow \mu \nu$ and $D_{s} \rightarrow l \nu$ ), the semi-leptonic transition $B \rightarrow D \tau \nu$, plus $B \rightarrow X_{s} \gamma$, including $B^{0}-\bar{B}^{0}$ mixing, $B_{s} \rightarrow \mu^{+} \mu^{-}$and the radiative decay $Z \rightarrow b \bar{b}$. Thirdly, having selected the surviving 2 HDM-III parameter space, we show that the $H^{-} c \bar{b}$ coupling can be very large over sizable expanses of it, in fact, a very different situation with respect to $2 \mathrm{HDM}$ with a flavor discrete symmetry (i.e., $\mathcal{Z}_{2}$ ) and very similar to the case of the Aligned-2HDM (A2HDM) as well as of models with three or more Higgs doublets. Fourthly, we study in detail the ensuing $H^{ \pm}$phenomenology at the Large Hadron Collider (LHC), chiefly the $c \bar{b} \rightarrow H^{+}$production mode and the $H^{+} \rightarrow c \bar{b}$ decay channel while assuming $\tau^{+} \nu_{\tau}$ decays in the former and $t \rightarrow b H^{+}$production in the latter, showing that significant scope exists in both cases.

Keywords: Higgs Physics, Beyond Standard Model

ARXIV EPRINT: 1212.6818 


\section{Contents}

1 Introduction 1

2 The Yukawa sector of the 2HDM-III with a four-zero Yukawa texture 4

2.1 The general Higgs potential in the 2HDM-III 4

2.2 The Yukawa sector in the 2HDM-III with a four-zero texture 6

3 Flavor constraints on the 2HDM-III with a four-zero Yukawa texture 12

$3.1 \mu-e$ universality in $\tau$ decays $\quad 12$

$\begin{array}{ll}3.2 & \text { Leptonic meson decays } \\ 3.3 & \text { Semileptonic }\end{array}$

$\begin{array}{ll}3.3 & \text { Semileptonic decays } B \rightarrow D \tau \nu \\ 3.4 & 16\end{array}$

$\begin{array}{lll}3.4 B \rightarrow X_{s} \gamma \text { decays } & 19\end{array}$

3.4.1 NLO Wilson coefficients at the scale $\mu_{W} \quad 20$

$\begin{array}{lll}3.5 & \mathrm{BR}\left(B \rightarrow X_{s} \gamma\right) & 21\end{array}$

$\begin{array}{lll}3.6 & B^{0}-\bar{B}^{0} \text { mixing } & 22\end{array}$

$3.7 Z \rightarrow b \bar{b} \quad 24$

$\begin{array}{lll}3.8 B_{s} \rightarrow \mu^{+} \mu^{-} & 25\end{array}$

4 Light charged Higgs boson phenomenology 27

4.1 The dominance of the $\operatorname{BR}\left(H^{ \pm} \rightarrow c b\right) \quad 29$

4.2 The decay $H^{ \pm} \rightarrow A W^{*}$ for $m_{A^{0}}<m_{H^{ \pm}}$

5 Numerical Results $\quad 35$

$\begin{array}{lll}5.1 & \text { Indirect } H^{ \pm} \text {production } & 35\end{array}$

5.2 Direct $H^{ \pm}$production 36

6 Conclusions $\quad 40$

\section{Introduction}

The main problem in flavor physics Beyond the Standard Model (BSM) [1] is to control the presence of Flavor Changing Neutral Currents (FCNCs) that have been observed to be highly suppressed by a variety of experiments. Almost all BSM scenarios that describe physics in energy regions higher than the Electro-Weak (EW) scale have contributions with FCNCs at tree level, unless some symmetry is introduced in the scalar sector to suppress them. One of the most important extensions of the SM is the 2-Higgs Doublet Model $(2 \mathrm{HDM})$ [2-4], due to its wide variety of dynamical features and the fact that it can represent a low-energy limit of general models like the Minimal Supersymmetric Standard Model (MSSM). There are several realizations of the 2HDM, called Type I, II, $\mathrm{X}$ and Y (acronymed as 2HDM-I [5], 2HDM-II [6], 2HDM-X and 2HDM-Y [7-10]) or 
inert Types, wherein (part of) the scalar particle content does not acquire a Vacuum Expectation Value (VEV) [11-14]. In the most general version of a $2 \mathrm{HDM}$, the fermionic couplings of the neutral scalars are non-diagonal in flavor and, therefore, generate unwanted FCNC phenomena. Different ways to suppress FCNCs have been developed, giving rise to a variety of specific implementations of the $2 \mathrm{HDM}$. The simplest and most common approach is to impose a $\mathcal{Z}_{2}$ symmetry forbidding all non-diagonal terms in flavor space in the Lagrangian [15]. Depending on the charge assignments under this symmetry, the model is called Type I, II, X and Y or inert. There are other suggestions for the most general 2HDM: (i) the alignment in flavor space of the Yukawa couplings of the two scalar doublets, which guarantees the absence of tree-level FCNC interactions [16, 17]; (ii) the Lepton Flavor Violating (LFV) terms introduced as a deviation from the Model II Yukawa interactions in $[18,19]$; (iii) the 2HDM-III with a particular Yukawa 'texture', forcing the non-diagonal Yukawa couplings to be proportional to the geometric mean of the two fermion masses, $g_{i j} \propto \sqrt{m_{i} m_{j}} \chi_{i j}[20-24]^{1}$; (iv) recently, a Partially Aligned 2HDM (PA2HDM) was presented and a four-zero texture is employed therein too, so that this newly suggested scenario includes (i) and (iii) as particular cases [27].

Therefore, the mechanism through which the FCNCs are controlled defines the actual version of the model and the consequently different phenomenology that can be contrasted with experiment. In particular, we focus here on the version where the Yukawa couplings depend on the hierarchy of masses. This version is the one where the mass matrix has a fourzero texture form $[25,26]$. This matrix is based on the phenomenological observation that the off-diagonal elements must be small in order to dim the interactions that violate flavor, as experimental results show. Although the phenomenology of Yukawa couplings constrains the hierarchy of the mass matrix entries, it is not enough to determine the strength of the interaction with scalars. Another assumption on the Yukawa matrix is related to the additional Higgs doublet. In versions I and II a discrete symmetry is introduced on the Higgs doublets, fulfilled by the scalar potential, that leads to the vanishing of most of the free parameters. However, version III, having a richer phenomenology, requires a slightly more general scheme. Interesting phenomenological implications of $2 \mathrm{HDMs}$ with a four-zero texture for the charged Higgs boson sector $[22,23,28]$ and neutral Higgs boson sector $[29,30]$ have been studied. In these works one estimated the order of the parameters $\chi_{i j}=\mathcal{O}(1)$, including the off-diagonal terms of the Yukawa texture. In contrast, a complete and detailed analysis that includes off-diagonal terms of the Yukawa texture in presence of the recent data of processes at low energy has been omitted in previous works. Therefore, in this paper, we present a comprehensive study of the main flavor constraints on the parameters that come from a four-zero Yukawa texture considering the off-diagonal terms and present their relevance for charged Higgs boson phenomenology at the Large Hadron Collider (LHC). The presence of a charged scalar $H^{ \pm}$is in fact one of the most distinctive features of a two-Higgs doublet extended scalar sector. In the following, we analyze its

\footnotetext{
${ }^{1}$ It is well known that, through Yukawa textures [25, 26], it is possible to build a matrix that preserves the expected Yukawa couplings that depend on the fermion masses. From a phenomenological point of view, the Cheng-Sher ansatz [20] has been very useful to describe the phenomenological content of the corresponding Yukawa matrix and the salient features of the hierarchy of quark masses.
} 
phenomenological impact in low-energy flavor-changing processes within the 2HDM-III with a four-zero texture and constrain the complex parameters $\chi_{i j}$ therein using present data on different leptonic, semi-leptonic/semi-hadronic and hadronic decays.

If $m_{H^{ \pm}}<m_{t}-m_{b}$, such particles would most copiously (though not exclusively [31,32]) be produced in the decays of top quarks via $t \rightarrow H^{ \pm} b$ [33]. Searches in this channel have been performed by the Tevatron experiments, assuming the decay modes $H^{ \pm} \rightarrow c s$ and $H^{ \pm} \rightarrow \tau \nu[34,35]$. Since no signal has been observed, constraints are obtained on the parameter space $\left[m_{H^{ \pm}}, \tan \beta\right]$, where $\tan \beta=v_{2} / v_{1}$ (i.e., the ratio of the VEVs of the two Higgs doublets). Searches in these channels have now been carried out also at the LHC: for $H^{ \pm} \rightarrow c s$ with $0.035 \mathrm{fb}^{-1}$ by ATLAS [36] and for $H^{ \pm} \rightarrow \tau \nu$ with $4.8 \mathrm{fb}^{-1}$ by ATLAS [37] plus with $1 \mathrm{fb}^{-1}$ by CMS [38]. These are the first searches for $H^{ \pm}$states at this collider. The constraints on $\left[m_{H^{ \pm}}, \tan \beta\right]$ from the LHC searches for $t \rightarrow H^{ \pm} b$ are now more restrictive than those obtained from the corresponding Tevatron searches.

The phenomenology of $H^{ \pm}$states in models with three or more Higgs doublets, called Multi-Higgs Doublet Models (MHDMs), was first studied comprehensively in [8], with an emphasis on the constraints from low-energy processes (e.g., the decays of mesons). Although the phenomenology of $H^{ \pm}$bosons at high-energy colliders in MHDMs and 2HDMs has many similarities, the possibility of $m_{H^{ \pm}}<m_{t}-m_{b}$ together with an enhanced Branching Ratio (BR) for $H^{ \pm} \rightarrow c b$ would be a distinctive feature of MHDMs. This scenario, which was first mentioned in [8] and studied in more detail originally in $[10,39,40]$ and most recently in [41], is of immediate interest for the ongoing searches for $t \rightarrow H^{ \pm} b$ with $H^{ \pm} \rightarrow c s$ by the LHC [36]. Although the current limits on $H^{ \pm} \rightarrow c s$ can also be applied to the decay $H^{ \pm} \rightarrow c b$ (as discussed in [42] in the context of the Tevatron searches), a further improvement in sensitivity to $t \rightarrow H^{ \pm} b$ with $H^{ \pm} \rightarrow c b$ could be obtained by tagging the $b$ quark which originates from $H^{ \pm}$decays $[23,39,41,42]$. Large values of $\operatorname{BR}\left(H^{ \pm} \rightarrow c b\right)$ are also possible in certain 2HDMs, such as the "flipped 2HDM" with Natural Flavor Conservation (NFC) $[4,10,42]$. However, in this model one would generally expect $m_{H^{ \pm}} \gg m_{t}$, due to the constraint from $b \rightarrow s \gamma\left(m_{H^{ \pm}}>295 \mathrm{GeV}[43,44]\right)$ so that $t \rightarrow H^{ \pm} b$ with $H^{ \pm} \rightarrow c b$ would not proceed. However, in our version of the 2 HDM-III there are additional new physics contributions which enter $b \rightarrow s \gamma$, thus weakening the constraint on $m_{H^{ \pm}}$[23]. We will estimate the increase in sensitivity to $\operatorname{BR}\left(H^{ \pm} \rightarrow c b\right)$ and to the fermionic couplings of $H^{ \pm}$in the 2HDM-III scenario. Further, always in the latter, we will re-visit the possibility of direct $H^{ \pm}$production from $c b$-fusion, where the on-shell $H^{ \pm}$state eventually decays to $\tau \nu_{\tau}$ pairs, its only resolvable signature in the context of fully hadronic machines.

We now proceed as follows. The formulation of the general 2HDM with a four-zero texture for the Yukawa matrix is recalled in section 2. The phenomenological consequences of having a charged Higgs field are analyzed in the next section in processes at low energy, extracting the corresponding constraints on the aforementioned new physics parameters $\chi_{i j}$, by discussing the constraints derived from tree-level leptonic and semi-leptonic/semihadronic decays, while in section IV we discuss the ensuing light charged Higgs phenomenology at the LHC. Finally, we elaborate our conclusions in section V. 


\section{The Yukawa sector of the 2HDM-III with a four-zero Yukawa texture}

In this section, we will discuss the main characteristics of the general Higgs potential and the using of a specific four-zero texture in the Yukawa matrices within the 2HDM-III. In this connection, notice that, when a flavor symmetry in the Yukawa sector is implemented, discrete symmetries in the Higgs potential are not needed, so that the most general Higgs potential must be introduced.

\subsection{The general Higgs potential in the 2HDM-III}

The 2HDM includes two Higgs scalar doublets of hypercharge $+1: \Phi_{1}^{\dagger}=\left(\phi_{1}^{-}, \phi_{1}^{0 *}\right)$ and $\Phi_{2}^{\dagger}=\left(\phi_{2}^{-}, \phi_{2}^{0 *}\right)$. The most general $S U(2)_{L} \times U(1)_{Y}$ invariant scalar potential can be written as [45]

$$
\begin{aligned}
V\left(\Phi_{1}, \Phi_{2}\right)= & \mu_{1}^{2}\left(\Phi_{1}^{\dagger} \Phi_{1}\right)+\mu_{2}^{2}\left(\Phi_{2}^{\dagger} \Phi_{2}\right)-\left(\mu_{12}^{2}\left(\Phi_{1}^{\dagger} \Phi_{2}\right)+\text { H.c. }\right)+\frac{1}{2} \lambda_{1}\left(\Phi_{1}^{\dagger} \Phi_{1}\right)^{2} \\
& +\frac{1}{2} \lambda_{2}\left(\Phi_{2}^{\dagger} \Phi_{2}\right)^{2}+\lambda_{3}\left(\Phi_{1}^{\dagger} \Phi_{1}\right)\left(\Phi_{2}^{\dagger} \Phi_{2}\right)+\lambda_{4}\left(\Phi_{1}^{\dagger} \Phi_{2}\right)\left(\Phi_{2}^{\dagger} \Phi_{1}\right) \\
& +\left(\frac{1}{2} \lambda_{5}\left(\Phi_{1}^{\dagger} \Phi_{2}\right)^{2}+\left(\lambda_{6}\left(\Phi_{1}^{\dagger} \Phi_{1}\right)+\lambda_{7}\left(\Phi_{2}^{\dagger} \Phi_{2}\right)\right)\left(\Phi_{1}^{\dagger} \Phi_{2}\right)+\text { H.c. }\right)
\end{aligned}
$$

where all parameters are assumed to be real ${ }^{2}$. Regularly, in the 2HDM Type I and II the terms proportional to $\lambda_{6}$ and $\lambda_{7}$ are absent, because the discrete symmetry $\Phi_{1} \rightarrow \Phi_{1}$ and $\Phi_{2} \rightarrow-\Phi_{2}$ is imposed in order to avoid dangerous FCNC effects. However, in our model, where mass matrices with a four-zero texture are considered, as intimated, it is not necessary to implement the above discrete symmetry. Thence, one must keep the terms proportional to $\lambda_{6}$ and $\lambda_{7}$. These parameters play an important role in one-loop processes, where self-interactions of Higgs bosons could be relevant [30]. Besides, the parameters $\lambda_{6}$ and $\lambda_{7}$ are essential to obtain the decoupling limit of the model in which only one CP-even scalar is light, as hinted by current Tevatron and LHC data [46]. While these terms exist, there are two independent energy scales, $v$ and $\Lambda_{2 \mathrm{HDM}}$ (the scale at which additional BSM physics is required to control persisting divergences in the Higgs masses and self-couplings), and the spectrum of Higgs boson masses is such that $m_{h^{0}}$ is of order $v$ whilst $m_{H^{0}}, m_{A^{0}}$ and $m_{H^{ \pm}}$are all of the order of $\Lambda_{2 \mathrm{HDM}}$ [45]. Then, the heavy Higgs bosons decouple in the limit $\Lambda_{2 \mathrm{HDM}} \gg v$, according to the decoupling theorem [3]. Conversely, when the scalar potential does respect the discrete symmetry, it is impossible to have two independent energy scales [45]. This implies that all of the physical scalar masses lie at the EW scale $v$. Being that $v$ is already fixed by experiment though, a very heavy Higgs boson can only arise by means of a large dimensionless coupling constant $\lambda_{i}$. In this case, the decoupling theorem is not valid, thus opening the possibility for the appearance of non-decoupling effects. Moreover, since the scalar potential contains some terms that violate the $S U(2)$ custodial symmetry, non-decoupling effects can arise in one-loop induced Higgs boson couplings [47].

The scalar potential (2.2) has been diagonalized to generate the mass-eigenstates fields. The charged components of the doublets lead to a physical charged Higgs boson and the

\footnotetext{
${ }^{2}$ The $\mu_{12}^{2}, \lambda_{5}, \lambda_{6}$ and $\lambda_{7}$ parameters are complex in general, but we will assume that they are real for simplicity.
} 
pseudo-Goldstone boson associated with the $W$ gauge field:

$$
\begin{aligned}
& G_{W}^{ \pm}=\phi_{1}^{ \pm} c_{\beta}+\phi_{2}^{ \pm} s_{\beta}, \\
& H^{ \pm}=-\phi_{1}^{ \pm} s_{\beta}+\phi_{2}^{ \pm} c_{\beta},
\end{aligned}
$$

with

$$
m_{H^{ \pm}}^{2}=\frac{\mu_{12}^{2}}{s_{\beta} c_{\beta}}-\frac{1}{2} v^{2}\left(\lambda_{4}+\lambda_{5}+t_{\beta}^{-1} \lambda_{6}+t_{\beta} \lambda_{7}\right)
$$

where we have introduced the short-hand notations, $t_{\beta}=\tan \beta, s_{\beta}=\sin \beta$ and $c_{\beta}=\cos \beta$. Besides, the imaginary part of the neutral components $\phi_{i I}^{0}$ defines the neutral CP-odd state and the pseudo-Goldstone boson associated with the $Z$ gauge boson. The corresponding rotation is given by:

$$
\begin{aligned}
& G_{Z}=\phi_{1 I}^{0} c_{\beta}+\phi_{2 I}^{0} s_{\beta}, \\
& A^{0}=-\phi_{1 I}^{0} s_{\beta}+\phi_{2 I}^{0} c_{\beta},
\end{aligned}
$$

where

$$
m_{A^{0}}^{2}=m_{H^{ \pm}}^{2}+\frac{1}{2} v^{2}\left(\lambda_{4}-\lambda_{5}\right)
$$

Finally, the real part of the neutral components of the $\phi_{i R}^{0}$ doublets defines the CP-even Higgs bosons $h^{0}$ and $H^{0}$. The mass matrix is given by:

$$
M_{R e}=\left(\begin{array}{ll}
m_{11} & m_{12} \\
m_{12} & m_{22}
\end{array}\right) \text {, }
$$

where

$$
\begin{aligned}
& m_{11}=m_{A}^{2} s_{\beta}^{2}+v^{2}\left(\lambda_{1} c_{\beta}^{2}+s_{\beta}^{2} \lambda_{5}+2 s_{\beta} c_{\beta} \lambda_{6}\right), \\
& m_{22}=m_{A}^{2} c_{\beta}^{2}+v^{2}\left(\lambda_{2} s_{\beta}^{2}+c_{\beta}^{2} \lambda_{5}+2 s_{\beta} c_{\beta} \lambda_{7}\right), \\
& m_{12}=-m_{A}^{2} s_{\beta} c_{\beta}+v^{2}\left(\left(\lambda_{3}+\lambda_{4}\right) s_{\beta} c_{\beta}+\lambda_{6} c_{\beta}^{2}+\lambda_{7} s_{\beta}^{2}\right) .
\end{aligned}
$$

The physical CP-even states, $h^{0}$ and $H^{0}$, are written as

$$
\begin{aligned}
& H^{0}=\phi_{1 R}^{0} c_{\alpha}+\phi_{2 R}^{0} s_{\alpha}, \\
& h^{0}=-\phi_{1 R}^{0} s_{\alpha}+\phi_{2 R}^{0} c_{\alpha},
\end{aligned}
$$

where

$$
\tan 2 \alpha=\frac{2 m_{12}}{m_{11}-m_{22}}
$$

and

$$
m_{H^{0}, h^{0}}^{2}=\frac{1}{2}\left(m_{11}+m_{22} \pm \sqrt{\left(m_{11}-m_{22}\right)^{2}+4 m_{12}^{2}}\right) .
$$




\subsection{The Yukawa sector in the 2HDM-III with a four-zero texture}

We shall follow Refs. [22, 48], where a specific four-zero texture has been implemented for the Yukawa matrices within the 2HDM-III. This allows one to express the couplings of the neutral and charged Higgs bosons in terms of the fermion masses, Cabibbo-KobayashiMaskawa (CKM) mixing angles and certain dimensionless parameters, which are to be bounded by current experimental constraints. Thus, in order to derive the interactions of the charged Higgs boson, the Yukawa Lagrangian is written as follows:

$$
\begin{aligned}
\mathcal{L}_{Y} & =-\left(Y_{1}^{u} \bar{Q}_{L} \tilde{\Phi}_{1} u_{R}+Y_{2}^{u} \bar{Q}_{L} \tilde{\Phi}_{2} u_{R}+Y_{1}^{d} \bar{Q}_{L} \Phi_{1} d_{R}\right. \\
& \left.+Y_{2}^{d} \bar{Q}_{L} \Phi_{2} d_{R}+Y_{1}^{l} \overline{L_{L}} \Phi_{1} l_{R}+Y_{2}^{l} \overline{L_{L}} \Phi_{2} l_{R}\right),
\end{aligned}
$$

where $\Phi_{1,2}=\left(\phi_{1,2}^{+}, \phi_{1,2}^{0}\right)^{T}$ refer to the two Higgs doublets, $\tilde{\Phi}_{1,2}=i \sigma_{2} \Phi_{1,2}^{*}, Q_{L}$ denotes the left-handed fermion doublet, $u_{R}$ and $d_{R}$ are the right-handed fermion singlets and, finally, $Y_{1,2}^{u, d}$ denote the $(3 \times 3)$ Yukawa matrices. Similarly, one can see the corresponding lefthanded fermion doublet $L_{L}$, the right-handed fermion singlet $l_{R}$ and the Yukawa matrices $Y_{1,2}^{l}$ for leptons.

After spontaneous EW Symmetry Breaking (EWSB), one can derive the fermion mass matrices from eq. (2.16), namely

$$
M_{f}=\frac{1}{\sqrt{2}}\left(v_{1} Y_{1}^{f}+v_{2} Y_{2}^{f}\right), \quad f=u, d, l .
$$

We will assume that both Yukawa matrices $Y_{1}^{f}$ and $Y_{2}^{f}$ have the four-texture form and are Hermitian $[22,26]$. Following this convention, the fermions mass matrices have the same form, which can be written as:

$$
M_{f}=\left(\begin{array}{ccc}
0 & C_{f} & 0 \\
C_{f}^{*} & \tilde{B}_{f} & B_{f} \\
0 & B_{f}^{*} & A_{f}
\end{array}\right) .
$$

When $\tilde{B}_{q} \rightarrow 0$ one recovers the six-texture form. We also consider the hierarchy $\left|A_{q}\right| \gg \mid$ $\tilde{B}_{q}|,| B_{q}|,| C_{q} \mid$, which is supported by the observed fermion masses in the SM.

The mass matrix is diagonalized through the bi-unitary matrices $V_{L, R}$, though each Yukawa matrices is not diagonalized by this transformation. The diagonalization is performed in the following way:

$$
\bar{M}_{f}=V_{f L}^{\dagger} M_{f} V_{f R}
$$

The fact that $M_{f}$ is Hermitian, under the considerations given above, directly implies that $V_{f L}=V_{f R}$, and the mass eigenstates for the fermions are given by

$$
u=V_{u}^{\dagger} u^{\prime}, \quad d=V_{d}^{\dagger} d^{\prime}, \quad l=V_{l}^{\dagger} l^{\prime} .
$$

Then, eq. (2.17) in this basis takes the form

$$
\bar{M}_{f}=\frac{1}{\sqrt{2}}\left(v_{1} \tilde{Y}_{1}^{f}+v_{2} \tilde{Y}_{2}^{f}\right)
$$


where $\tilde{Y}_{i}^{f}=V_{f L}^{\dagger} Y_{i}^{f} V_{f R}$. In order to compare the kind of new physics coming from our Yukawa texture with some more traditional 2HDMs (in particular with the 2HDM-II), in previous works [22, 23, 28-30], some of us have adopted the following re-definitions:

2HDM-II-like

$$
\begin{aligned}
\tilde{Y}_{1}^{d} & =\frac{\sqrt{2}}{v \cos \beta} \bar{M}_{d}-\tan \beta \tilde{Y}_{2}^{d}, \\
\tilde{Y}_{2}^{u} & =\frac{\sqrt{2}}{v \sin \beta} \bar{M}_{u}-\cot \beta \tilde{Y}_{1}^{u}, \\
\tilde{Y}_{1}^{l} & =\tilde{Y}_{1}^{d}(d \rightarrow l) .
\end{aligned}
$$

These re-definitions are convenient because we can get the Higgs-fermion-fermion coupling in the 2HDM-III as $g_{2 \mathrm{HDM}-\mathrm{III}}^{f f \phi}=g_{2 \mathrm{HDM}-\mathrm{II}}^{f f \phi}+\Delta g^{f f \phi}$, where $g_{2 \mathrm{HDM}-\mathrm{II}}^{f f \phi}$ is the coupling in the 2HDM-II and $\Delta g^{f f \phi}$ is the contribution of the four-zero texture. If $\Delta g^{f f \phi} \rightarrow 0$ we can recover the 2HDM-II. However, these re-definitions are not unique. In fact, there are others possibilities since from eq. (2.21) one can reproduce the 2HDM-I, 2HDM-X or $2 \mathrm{HDM}-\mathrm{Y}$ as we can obtain for any version of $2 \mathrm{HDM}$ the following relation: $g_{2 \mathrm{HDM}-\mathrm{III}}^{f f \phi}=$ $g_{2 \mathrm{HDM}-\text { any }}^{f f \phi}+\Delta^{\prime} g^{f f \phi}$. The other possible re-definitions are:

2HDM-I-like

$$
\begin{aligned}
\tilde{Y}_{2}^{d} & =\frac{\sqrt{2}}{v \sin \beta} \bar{M}_{d}-\cot \beta \tilde{Y}_{1}^{d}, \\
\tilde{Y}_{2}^{u} & =\frac{\sqrt{2}}{v \sin \beta} \bar{M}_{u}-\cot \beta \tilde{Y}_{1}^{u}, \\
\tilde{Y}_{2}^{l} & =\tilde{Y}_{2}^{d}(d \rightarrow l) .
\end{aligned}
$$

2HDM-X-like

$$
\begin{aligned}
\tilde{Y}_{2}^{d} & =\frac{\sqrt{2}}{v \sin \beta} \bar{M}_{d}-\cot \beta \tilde{Y}_{1}^{d}, \\
\tilde{Y}_{2}^{u} & =\frac{\sqrt{2}}{v \sin \beta} \bar{M}_{u}-\cot \beta \tilde{Y}_{1}^{u}, \\
\tilde{Y}_{1}^{l} & =\tilde{Y}_{1}^{d}(d \rightarrow l) .
\end{aligned}
$$

2HDM-Y-like

$$
\begin{aligned}
\tilde{Y}_{1}^{d} & =\frac{\sqrt{2}}{v \cos \beta} \bar{M}_{d}-\tan \beta \tilde{Y}_{2}^{d}, \\
\tilde{Y}_{2}^{u} & =\frac{\sqrt{2}}{v \sin \beta} \bar{M}_{u}-\cot \beta \tilde{Y}_{1}^{u}, \\
\tilde{Y}_{2}^{l} & =\tilde{Y}_{2}^{d}(d \rightarrow l) .
\end{aligned}
$$

After spontaneous EWSB and including the diagonalizing matrices for quarks and Higgs bosons ${ }^{3}$, the interactions of the charged Higgs bosons $H^{ \pm}$and neutral Higgs bosons $\phi^{0}\left(\phi^{0}=h^{0}, H^{0}, A^{0}\right)$ with quark pairs for any parametrization 2HDM-(I,II,X,Y)-like have

\footnotetext{
${ }^{3}$ The details of both diagonalizations are presented in Ref. [22].
} 
the following form:

$$
\begin{aligned}
\mathcal{L}^{\bar{f}_{i} f_{j} \phi}= & -\frac{g}{2 \sqrt{2} M_{W}}\left[\sum _ { l = 1 } ^ { 3 } \overline { u } _ { i } \left\{\left(V_{\mathrm{CKM}}\right)_{i l}\left[X m_{d_{l}} \delta_{l j}-f(X)\left(\frac{\sqrt{2} M_{W}}{g}\right)\left(\tilde{Y}_{n(X)}^{d}\right)_{l j}\right]\left(1+\gamma^{5}\right)\right.\right. \\
& \left.+\left[Y m_{u_{i}} \delta_{i l}-f(Y)\left(\frac{\sqrt{2} M_{W}}{g}\right)\left(\tilde{Y}_{n(Y)}^{u}\right)_{i l}^{\dagger}\right]\left(V_{\mathrm{CKM}}\right)_{l j}\left(1-\gamma^{5}\right)\right\} d_{j} H^{+} \\
& \left.+\bar{\nu}_{i}\left[Z m_{l_{i}} \delta_{i j}-f(Z)\left(\frac{\sqrt{2} M_{W}}{g}\right)\left(\tilde{Y}_{n(Z)}^{l}\right)_{i j}\right]\left(1+\gamma^{5}\right) l_{j} H^{+}+h . c .\right] \\
& -\frac{g}{2 M_{W}}\left(m _ { d _ { i } } \overline { d } _ { i } \left\{\left[\xi_{H}^{d} \delta_{i j}-\frac{\left(\xi_{h}^{d}+X \xi_{H}^{d}\right)}{f(X)} \frac{\sqrt{2}}{g}\left(\frac{m_{W}}{m_{d_{i}}}\right)\left(\tilde{Y}_{n(X)}^{d}\right)_{i j}\right] H^{0}\right.\right. \\
& +\left[\xi_{h}^{d} \delta_{i j}+\frac{\left(\xi_{H}^{d}-X \xi_{h}^{d}\right)}{f(X)} \frac{\sqrt{2}}{g}\left(\frac{m_{W}}{m_{d_{i}}}\right)\left(\tilde{Y}_{n(X)}^{d}\right)_{i j}\right] h^{0} \\
& \left.+i\left[-X \delta_{i j}+f(X) \frac{\sqrt{2}}{g}\left(\frac{m_{W}}{m_{d_{i}}}\right)\left(\tilde{Y}_{n(X)}^{d}\right)_{i j}\right] \gamma^{5} A^{0}\right\} d_{j} \\
& +m_{u_{i}} \bar{u}_{i}\left\{\left[\xi_{H}^{u} \delta_{i j}+\frac{\left(\xi_{h}^{u}-Y \xi_{H}^{u}\right)}{f(Y)} \frac{\sqrt{2}}{g}\left(\frac{m_{W}}{m_{u_{i}}}\right)\left(\tilde{Y}_{n(Y)}^{u}\right)_{i j}\right] H^{0}\right. \\
& +\left[\xi_{h}^{u} \delta_{i j}-\frac{\left(\xi_{H}^{u}+Y \xi_{h}^{u}\right)}{f(Y)} \frac{\sqrt{2}}{g}\left(\frac{m_{W}}{m_{u_{i}}}\right)\left(\tilde{Y}_{n(Y)}^{u}\right)_{i j}\right] h^{0} \\
& \left.+i\left[-Y \delta_{i j}+f(Y) \frac{\sqrt{2}}{g}\left(\frac{m_{W}}{m_{u_{i}}}\right)\left(\tilde{Y}_{n(Y)}^{u}\right)_{i j}\right] \gamma^{5} A^{0}\right\} u_{j} \\
& +m_{l_{i}} \bar{l}_{i}\left\{\left[\xi_{H}^{l} \delta_{i j}-\frac{\left(\xi_{h}^{l}+Z \xi_{H}^{l}\right)}{f(Z)} \frac{\sqrt{2}}{g}\left(\frac{m_{W}}{m_{d_{i}}}\right)\left(\tilde{Y}_{n(Z)}^{l}\right)_{i j}\right] H^{0}\right. \\
& +\left[\xi_{h}^{l} f(Z) \delta_{i j}+\frac{\left(\xi_{H}^{l}-Z \xi_{h}^{l}\right)}{f(Z)} \frac{\sqrt{2}}{g}\left(\frac{m_{W}}{m_{d_{i}}}\right)\left(\tilde{Y}_{n(Z)}^{l}\right)_{i j}\right] h^{0} \\
& \left.\left.+i\left[-Z \delta_{i j}+f(Z) \frac{\sqrt{2}}{g}\left(\frac{m_{W}}{m_{d_{i}}}\right)\left(\tilde{Y}_{n(Z)}^{l}\right)_{i j}\right] \gamma^{5} A^{0}\right\} l_{j}\right), \\
& {\left[y_{0}\right) }
\end{aligned}
$$

where $V_{\mathrm{CKM}}$ denotes the mixing matrices of the quark sector, the functions $f_{(}(x)$ and $n(x)$ are given by:

$$
\begin{aligned}
& f(x)=\sqrt{1+x^{2}}, \\
& n(x)=\left\{\begin{array}{l}
2 \text { if } x=\tan \beta, \\
1 \text { if } x=\cot \beta .
\end{array}\right.
\end{aligned}
$$

the parameters $X, Y, Z$ are given in Refs. $[4,8-10,16,41,43]$ and the factors $\xi_{\phi}^{f}$ are presented in Ref. [4]. Following this notation we can list the parameters for the framework 2HDM-(I,II,X,Y)-like through Tab. 1.

Following the analysis in [22] one can derive a better approximation for the product $V_{q} Y_{n}^{q} V_{q}^{\dagger}$, expressing the rotated matrix $\tilde{Y}_{n}^{q}$, in the form

$$
\left[\tilde{Y}_{n}^{q}\right]_{i j}=\frac{\sqrt{m_{i}^{q} m_{j}^{q}}}{v}\left[\tilde{\chi}_{n}^{q}\right]_{i j}=\frac{\sqrt{m_{i}^{q} m_{j}^{q}}}{v}\left[\chi_{n}^{q}\right]_{i j} e^{i \vartheta_{i j}^{q}},
$$


where the $\chi$ 's are unknown dimensionless parameters of the model, they come from the election of a specific texture of the Yukawa matrices. It is important to mention that eq. (2.28) is a consequence of the diagonalization process of Yuwaka matrices, assuming the hierarchy among the fermion masses (see Ref. [22]), namely, the Cheng-Sher ansatz is a particular case of this parametrization. Besides, in order to have an acceptable model, the parameters $\chi$ 's could be $O(1)$ but not more, generally. Recently we have calculated the $\chi^{2}$ fit of Yukawa matrices including the CKM matrix, and we find that the parameters off-diagonal are $O(1)$ (e.g., $\left.\chi_{23}^{f} \leq 10\right)$, therefore we cannot ignore all of these [49]. Besides, in Ref. [50], they study the general $2 \mathrm{HDMs}$ considering renormalization group evolution of the Yukawa couplings and the cases when the $\mathcal{Z}_{2}$-symmetry is broken, called non-diagonal models (e.g., the models with a structure incorporating the Cheng-Sher ansatz). It is interesting to note that it is actually the off-diagonal elements in the down-sector that become large whereas the ones in the up-sector $\chi^{u}(\mu) \leq 0.1$, assuming the conservative criterion $\chi^{f} \leq 0.1$, where $\mu$ is the renormalization scale. On the other hand, the FCNC processes at low energy are going to determine bounds for these parameters with high precision, aspect which is studied in this work. In order to perform our phenomenological study, we find it convenient to rewrite the Lagrangian given in eq. (2.26) in terms of the coefficients $\left[\tilde{\chi}_{n}^{q}\right]_{i j}$, as follows:

$$
\begin{aligned}
\mathcal{L}^{\bar{f}_{i} f_{j} \phi}= & -\frac{g}{2 \sqrt{2} M_{W}}\left[\sum _ { l = 1 } ^ { 3 } \overline { u } _ { i } \left[\left(V_{\mathrm{CKM}}\right)_{i l}\left(X m_{d_{l}} \delta_{l j}-\frac{f(X)}{\sqrt{2}} \sqrt{m_{d_{l}} m_{d_{j}}} \tilde{\chi}_{l j}^{d}\right)\left(1+\gamma^{5}\right)\right.\right. \\
& \left.+\left(Y m_{u_{i}} \delta_{i l}-\frac{f(Y)}{\sqrt{2}} \sqrt{m_{u_{i}} m_{u_{l}}} \tilde{\chi}_{i l}^{u}\right)\left(V_{\mathrm{CKM}}\right)_{l j}\left(1-\gamma^{5}\right)\right] d_{j} H^{+} \\
& \left.+\bar{\nu}_{i}\left(Z m_{l_{i}} \delta_{i j}-\frac{f(Z)}{\sqrt{2}} \sqrt{m_{l_{i}} m_{d_{j}}} \tilde{\chi}_{i j}^{l}\right)\left(1+\gamma^{5}\right) l_{j} H^{+}+h . c .\right] \\
& -\frac{g}{2 M_{W}}\left[\overline { d } _ { i } \left(\left[m_{d_{i}} \xi_{H}^{d} \delta_{i j}-\frac{\left(\xi_{h}^{d}+X \xi_{H}^{d}\right)}{f(X)} \frac{\sqrt{m_{d_{i}} m_{d_{j}}}}{\sqrt{2}} \tilde{\chi}_{i j}^{d}\right] H^{0}\right.\right. \\
& +\left[m_{d_{i}} \xi_{h}^{d} \delta_{i j}+\frac{\left(\xi_{H}^{d}-X \xi_{h}^{d}\right)}{f(X)} \frac{\sqrt{m_{d_{i}} m_{d_{j}}}}{\sqrt{2}} \tilde{\chi}_{i j}^{d}\right] h^{0} \\
& \left.+i\left[-m_{d_{i}} X \delta_{i j}+f(X) \frac{\sqrt{m_{d_{i}} m_{d_{j}}}}{\sqrt{2}} \tilde{\chi}_{i j}^{d}\right] \gamma^{5} A^{0}\right) d_{j} \\
& u_{i}\left(\left[m_{u_{i}} \xi_{H}^{u} \delta_{i j}+\frac{\left(\xi_{h}^{u}-Y \xi_{H}^{u}\right)}{f(Y)} \frac{\sqrt{m_{u_{i}} m_{u_{j}}}}{\sqrt{2}} \tilde{\chi}_{i j}^{u}\right] H^{0}\right. \\
& +\left[m_{u_{i}} \xi_{h}^{u} \delta_{i j}-\frac{\left(\xi_{H}^{u}+Y \xi_{h}^{u}\right)}{f(Y)}\left(\frac{\sqrt{m_{u_{i}} m_{u_{j}}}}{\sqrt{2}}\right) \tilde{\chi}_{i j}^{u}\right] h^{0} \\
& +i\left[-m_{u_{i}} Y \delta_{i j}+f(Y) \frac{\left.\left.\sqrt{m_{u_{i}} m_{u_{j}}} \tilde{\chi}_{i j}^{u}\right] \gamma^{5} A^{0}\right) u_{j}}{\sqrt{2}}\right. \\
& +\bar{l}_{i}\left(\left[m_{l_{i}} \xi_{H}^{l} \delta_{i j}-\frac{\left(\xi_{h}^{l}+Z \xi_{H}^{l}\right)}{f(Z)} \frac{\sqrt{m_{l_{i}} m_{l_{j}}}}{\sqrt{2}} \tilde{\chi}_{i j}^{l}\right] H^{0}\right. \\
& +\left[m_{l_{i}} \xi_{h}^{l} \delta_{i j}+\frac{\left(\xi_{H}^{l}-Z \xi_{h}^{l}\right)}{f(Z)} \frac{\sqrt{m_{l_{i}} m_{l_{j}}}}{\sqrt{2}} \tilde{\chi}_{i j}^{l}\right] h^{0} \\
& +m_{l_{i}} Z \delta_{i j}+f(Z) \frac{\left.\left.\left.\sqrt{m_{l_{i}} m_{l_{j}}} \tilde{\chi}_{i j}^{l}\right] \gamma^{5} A^{0}\right) l_{j}\right]}{\sqrt{2}},
\end{aligned}
$$


where we have redefined $\left[\tilde{\chi}_{1}^{u}\right]_{i j}=\tilde{\chi}_{i j}^{u},\left[\tilde{\chi}_{2}^{d}\right]_{i j}=\tilde{\chi}_{i j}^{d}$ and $\left[\tilde{\chi}_{2}^{l}\right]_{i j}=\tilde{\chi}_{i j}^{l}$. Then, from eq. (2.29), the couplings $\bar{f}_{i} f_{j} \phi^{0}, \bar{u}_{i} d_{j} H^{+}$and $u_{i} \bar{d}_{j} H^{-}$are given by:

$$
\begin{aligned}
g_{h^{0} \bar{f}_{i} f_{j}} & =-\frac{i g}{2 M_{W}}\left(m_{f_{i}} h_{i j}^{f}\right), \quad g_{H^{0} \bar{f}_{i} f_{j}}=-\frac{i g}{2 M_{W}}\left(m_{f_{i}} H_{i j}^{f}\right), \quad g_{A^{0} \bar{f}_{i} f_{j}}=-\frac{i g}{2 M_{W}}\left(m_{f_{i}} A_{i j}^{f} \gamma_{5}\right), \\
g_{H^{+} \bar{u}_{i} d_{j}} & =-\frac{i g}{2 \sqrt{2} M_{W}}\left(S_{i j}+P_{i j} \gamma_{5}\right), \quad g_{H^{-} u_{i} \overline{d_{j}}}=-\frac{i g}{2 \sqrt{2} M_{W}}\left(S_{i j}-P_{i j} \gamma_{5}\right) .
\end{aligned}
$$

where $h_{i j}^{f}, H_{i j}^{f}, A_{i j}^{f}, S_{i j}$ and $P_{i j}$ are defined as:

$$
\begin{aligned}
& h_{i j}^{d}=\xi_{h}^{d} \delta_{i j}+\frac{\left(\xi_{H}^{d}-X \xi_{h}^{d}\right)}{\sqrt{2} f(X)} \sqrt{\frac{m_{d_{j}}}{m_{d_{i}}}} \tilde{\chi}_{i j}^{d}, \quad h_{i j}^{l}=h_{i j}^{d}(d \rightarrow l, X \rightarrow Z), \\
& H_{i j}^{d}=\xi_{H}^{d} \delta_{i j}-\frac{\left(\xi_{h}^{d}+X \xi_{H}^{d}\right)}{\sqrt{2} f(X)} \sqrt{\frac{m_{d_{j}}}{m_{d_{i}}}} \tilde{\chi}_{i j}^{d}, \quad H_{i j}^{l}=H_{i j}^{d}(d \rightarrow l, X \rightarrow Z), \\
& A_{i j}^{d}=-X \delta_{i j}+\frac{f(X)}{\sqrt{2}} \sqrt{\frac{m_{d_{j}}}{m_{d_{i}}}} \tilde{\chi}_{i j}^{d}, \quad A_{i j}^{l}=A_{i j}^{d}(d \rightarrow l, X \rightarrow Z), \\
& h_{i j}^{u}=\xi_{h}^{u} \delta_{i j}-\frac{\left(\xi_{H}^{u}+Y \xi_{h}^{u}\right)}{\sqrt{2} f(Y)} \sqrt{\frac{m_{u_{j}}}{m_{u_{i}}}} \tilde{\chi}_{i j}^{u}, \\
& H_{i j}^{u}=\xi_{H}^{u} \delta_{i j}+\frac{\left(\xi_{h}^{u}-Y \xi_{H}^{u}\right)}{\sqrt{2} f(Y)} \sqrt{\frac{m_{u_{j}}}{m_{u_{i}}}} \tilde{\chi}_{i j}^{u}, \\
& A_{i j}^{u}=-Y \delta_{i j}+\frac{f(Y)}{\sqrt{2}} \sqrt{\frac{m_{u_{j}}}{m_{u_{i}}}} \tilde{\chi}_{i j}^{u} \text {, } \\
& S_{i j}=m_{d_{j}} X_{i j}+m_{u_{i}} Y_{i j}, \quad P_{i j}=m_{d_{j}} X_{i j}-m_{u_{i}} Y_{i j},
\end{aligned}
$$

with

$$
\begin{aligned}
X_{i j} & =\sum_{l=1}^{3}\left(V_{\mathrm{CKM}}\right)_{i l}\left[X \frac{m_{d_{l}}}{m_{d_{j}}} \delta_{l j}-\frac{f(X)}{\sqrt{2}} \sqrt{\frac{m_{d_{l}}}{m_{d_{j}}}} \tilde{\chi}_{l j}^{d}\right], \\
Y_{i j} & =\sum_{l=1}^{3}\left[Y \delta_{i l}-\frac{f(Y)}{\sqrt{2}} \sqrt{\frac{m_{u_{l}}}{m_{u_{i}}}} \tilde{\chi}_{i l}^{u}\right]\left(V_{\mathrm{CKM}}\right)_{l j} .
\end{aligned}
$$

For the case of leptons $S_{i j}^{l}=P_{i j}^{l}$ we have

$$
\begin{aligned}
S_{i j}^{l} & =m_{l_{j}} Z_{i j}^{l}, \\
Z_{i j}^{l} & =\left[Z \frac{m_{l_{i}}}{m_{l_{j}}} \delta_{i j}-\frac{f(Z)}{\sqrt{2}} \sqrt{\frac{m_{l_{i}}}{m_{l_{j}}}} \tilde{\chi}_{i j}^{l}\right] .
\end{aligned}
$$

Then, the couplings $l_{i}^{-} \nu_{l_{j}} H^{+}$and $l_{i}^{+} \nu_{l_{j}} H^{-}$are given by

$$
g_{H^{+} l_{i}^{-} \nu_{l_{j}}}=-\frac{i g}{\sqrt{2} M_{W}} S_{i j}^{l}\left(\frac{1+\gamma_{5}}{2}\right), \quad g_{H^{-} l_{i}^{+} \nu_{l_{j}}}=-\frac{i g}{\sqrt{2} M_{W}} S_{i j}^{l}\left(\frac{1-\gamma_{5}}{2}\right) .
$$

In order to compare these couplings with previous works $[4,8-10,41,43]$, we find it convenient to define the couplings $\bar{u}_{i} d_{j} H^{+}$and $u_{i} \bar{d}_{j} H^{-}$in terms of the matrix elements $X_{i j}, Y_{i j}$ and $Z_{i j}$. Following the definitions (2.32)-(2.35) we obtain the following compact 


\begin{tabular}{|l|c|c|c|c|c|c|c|c|c|}
\hline \multicolumn{1}{|c|}{ 2HDM-III } & $X$ & $Y$ & $Z$ & $\xi_{h}^{u}$ & $\xi_{h}^{d}$ & $\xi_{l}^{d}$ & $\xi_{H}^{u}$ & $\xi_{H}^{d}$ & $\xi_{H}^{l}$ \\
\hline 2HDM-I-like & $-\cot \beta$ & $\cot \beta$ & $-\cot \beta$ & $c_{\alpha} / s_{\beta}$ & $c_{\alpha} / s_{\beta}$ & $c_{\alpha} / s_{\beta}$ & $s_{\alpha} / s_{\beta}$ & $s_{\alpha} / s_{\beta}$ & $s_{\alpha} / s_{\beta}$ \\
2HDM-II-like & $\tan \beta$ & $\cot \beta$ & $\tan \beta$ & $c_{\alpha} / s_{\beta}$ & $-s_{\alpha} / c_{\beta}$ & $-s_{\alpha} / c_{\beta}$ & $s_{\alpha} / s_{\beta}$ & $c_{\alpha} / c_{\beta}$ & $c_{\alpha} / c_{\beta}$ \\
2HDM-X-like & $-\cot \beta$ & $\cot \beta$ & $\tan \beta$ & $c_{\alpha} / s_{\beta}$ & $c_{\alpha} / s_{\beta}$ & $-s_{\alpha} / c_{\beta}$ & $s_{\alpha} / s_{\beta}$ & $s_{\alpha} / s_{\beta}$ & $c_{\alpha} / c_{\beta}$ \\
2HDM-Y-like & $\tan \beta$ & $\cot \beta$ & $-\cot \beta$ & $c_{\alpha} / s_{\beta}$ & $-s_{\alpha} / c_{\beta}$ & $c_{\alpha} / s_{\beta}$ & $s_{\alpha} / s_{\beta}$ & $c_{\alpha} / c_{\beta}$ & $s_{\alpha} / s_{\beta}$ \\
\hline
\end{tabular}

Table 1. Parameters $X, Y$ and $Z$ defined in the Yukawa interactions of eq. (2.26) for four versions of the 2HDM-III with a four-zero texture, which come from eqs. (2.22)-(2.25). Here $s_{\alpha}=\sin \alpha$, $c_{\alpha}=\cos \alpha, s_{\beta}=\sin \beta$ and $c_{\beta}=\cos \beta$.

expression for the interactions of Higgs bosons with the fermions:

$$
\begin{aligned}
\mathcal{L}^{\bar{f}_{i} f_{j} \phi}= & -\left\{\frac{\sqrt{2}}{v} \bar{u}_{i}\left(m_{d_{j}} X_{i j} P_{R}+m_{u_{i}} Y_{i j} P_{L}\right) d_{j} H^{+}+\frac{\sqrt{2} m_{l_{j}}}{v} Z_{i j} \overline{\nu_{L}} l_{R} H^{+}+H . c .\right\} \\
& -\frac{1}{v}\left\{\bar{f}_{i} m_{f_{i}} h_{i j}^{f} f_{j} h^{0}+\bar{f}_{i} m_{f_{i}} H_{i j}^{f} f_{j} H^{0}-i \bar{f}_{i} m_{f_{i}} A_{i j}^{f} f_{j} \gamma_{5} A^{0}\right\} .
\end{aligned}
$$

When the parameters $\chi_{i j}^{f}=0$, we obtain $X_{11}=X_{22}=X_{33}=X$ (similarly for $Y$ and $Z$ ) and one recovers the Yukawa interactions given in Refs. [4, 8-10,41]. Besides, in order to hold consistencies with the MHDM/A2HDM [8], we suggest that this Lagrangian could represent a MHDM/A2HDM with additional flavor physics in the Yukawa matrices as well as the possibility of FCNCs at tree level. Returning to the Lagrangian given in eq. (2.37), when the parameters $\chi_{i j}^{f}$ are present, one can see that $X_{11} \neq X_{22} \neq X_{33} \neq X$ and the criteria of flavor constraints on $X$ cannot be applied directly to $X_{i j}$ (the same for $Y$ and $Z$ ), but the analyses for low energy processes are similar. Below we shall discuss more about various aspects of the model. Finally, it should be pointed out that parameters $X$, $Y, Z, \xi_{\phi}^{f}$ and $\chi_{i j}$ are arbitrary complex numbers, opening the possibility of having new sources of CP violation with tree-level FCNCs.

Previously, in Ref. [24], the flavor constraints of the 2HDM-III with a six-zero texture were studied, finding interesting results that we can use. However, we should compare their results and ours so as to distinguish the two parametrizations. Firstly, the six-zero texture assumed in [24] has been disfavored by current data on the CKM mixing angles [26, 51]. Hence, we focus here onto the four-zero texture, which is still acceptable phenomenologically and of which we consider the non-diagonal terms of the Yukawa matrices. Secondly, in order to unify notations we relate the parameters $\lambda_{i j}^{F}$ of [24] with our parameters $X_{i j}$, $Y_{i j}$ and $Z_{i j}$ as given in eqs. (2.34) and (2.35), as follows ${ }^{4}$ :

$$
\begin{aligned}
\mathcal{L}^{\bar{f}_{i} f_{j} H^{+}}= & -\left\{\bar{u}_{i}\left(\sum_{l}^{3}\left(V_{\mathrm{CKM}}\right)_{i l} \rho_{l j}^{D} P_{R}-\sum_{l}^{3} \rho_{i l}^{U}\left(V_{\mathrm{CKM}}\right)_{l j} P_{L}\right) d_{j} H^{+}\right. \\
& \left.+\rho_{i j}^{l} \bar{\nu}_{L} l_{R} H^{+}+H . c .\right\}, \\
\rho_{i j}^{F}= & \frac{\sqrt{2 m_{F i} m_{F j}}}{v} \lambda i j^{F},
\end{aligned}
$$

\footnotetext{
${ }^{4}$ We adopt the description of the Yukawa sector presented in Ref. [24].
} 
where $\rho_{i j}^{F}$ was introduced following the Cheng-Sher ansatz, considering $\lambda_{i j}^{F} \sim O(1)$. If we compare this with eq. (2.37), after using eqs. (2.34) and (2.35), we obtain the following relations:

$$
\begin{aligned}
& \lambda_{i j}^{D}=\left[X \sqrt{\frac{m_{d_{i}}}{m_{d_{j}}}} \delta_{i j}-\frac{f(X)}{\sqrt{2}} \tilde{\chi}_{i j}^{d}\right], \\
& \lambda_{i j}^{U}=-\left[Y \sqrt{\frac{m_{u_{i}}}{m_{u_{j}}}} \delta_{i j}-\frac{f(X)}{\sqrt{2}} \tilde{\chi}_{i j}^{u}\right], \\
& \lambda_{i j}^{l}=\left[Z \sqrt{\frac{m_{l_{i}}}{m_{l_{j}}}} \delta_{i j}-\frac{f(X)}{\sqrt{2}} \tilde{\chi}_{i j}^{l}\right],
\end{aligned}
$$

and

$$
\begin{aligned}
X_{i j} & =\sum_{l}\left(V_{C K M}\right)_{i l} \sqrt{\frac{m_{d_{l}}}{m_{d_{j}}}} \lambda_{l j}^{D}, \\
Y_{i j} & =\sum_{l} \sqrt{\frac{m_{u_{l}}}{m_{u_{i}}}} \lambda_{i l}^{U}\left(V_{C K M}\right)_{l j} .
\end{aligned}
$$

In essence, in the remainder of our work, we assume that our model could represent an effective flavor theory, wherein the Higgs fields necessarily participates in the flavor structure and has the same features as those of renormalizable flavor models [52-55]. In this type of scenarios, a horizontal flavor symmetry, continuous or discrete, is added to the SM gauge group symmetry in such a way as to reproduce the observed mass and mixing angle patterns by only using renormalizable terms in the Lagrangians. This requirement has two immediate and interesting consequences: firstly, there must be more than one $S U(2)$ doublet scalar; secondly, at least some of them must transform non-trivially under the flavor symmetry $[56,57]$.

\section{Flavor constraints on the 2HDM-III with a four-zero Yukawa texture}

In this section we will analyze the most important FCNC processes that are sensitive to, in particular, charged Higgs boson exchange, the primary interest of this paper, as well as effect of a (neutral) SM-like Higgs boson $h^{0}$ (we assume that its mass is $m_{h^{0}}=125$ $\mathrm{GeV}$ ). Starting from measurements obtained from from these processes we constrain the new physics parameters $\chi_{i j}^{f}$ that come from four-zero Yukawa texture. Finally, we study the possibility of obtaining a light charged Higgs boson compatible with all such measurements. We will address the various experimental limits in different subsections.

\section{1 $\mu-e$ universality in $\tau$ decays}

The $\tau$ decays into $\mu \bar{\nu}_{\mu} \nu_{\tau}$ and $e \bar{\nu}_{e} \nu_{\tau}$ produce important constraints onto charged Higgs boson states coupling to leptons [58], through the requirement of $\mu-e$ universality. The consequent limits can be quantified through the following relation $[59,60]$ :

$$
\left(\frac{g_{\mu}}{g_{e}}\right)_{\tau}^{2}=\frac{B R\left(\tau \rightarrow \mu \bar{\nu}_{\mu} \nu_{\tau}\right)}{B R\left(\tau \rightarrow e \bar{\nu}_{e} \nu_{\tau}\right)} \frac{g\left(m_{e}^{2} / m_{\tau}^{2}\right)}{g\left(m_{\mu}^{2} / m_{\tau}^{2}\right)}=1.0036 \pm 0.0020
$$



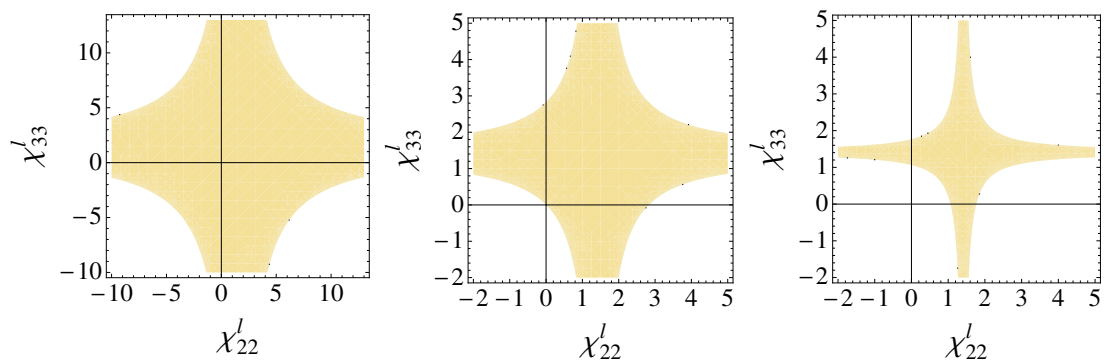

Figure 1. Considering the constraint from $\mu-e$ universality in $\tau$ decays, we show the allowed region (orange color) for $\chi_{22}^{l}$ and $\chi_{33}^{l}$ when $Z$ takes values of 10 (left-panel), 40 (center-panel) and 80 (right-panel). Here, $90 \mathrm{GeV} \leq m_{H^{ \pm}} \leq 130 \mathrm{GeV}$.

where $g(x)=1-8 x^{2}+8 x^{3}-x^{4}-12 x^{2} \log x$. Following [8], in our case, the request of $\mu-e$ universality imposes the following relation

$$
\frac{B R\left(\tau \rightarrow \mu \bar{\nu}_{\mu} \nu_{\tau}\right)}{B R\left(\tau \rightarrow e \bar{\nu}_{e} \nu_{\tau}\right)} \frac{f\left(m_{e}^{2} / m_{\tau}^{2}\right)}{f\left(m_{\mu}^{2} / m_{\tau}^{2}\right.} \simeq 1+\frac{R^{2}}{4}-0.25 R
$$

where $R$ is the scalar contribution parametrized through the effective coupling, see eqs. (2.35) and (2.36),

$$
R=\frac{m_{\tau} m_{\mu}}{m_{H^{ \pm}}^{2}} Z_{33} Z_{22}=\frac{m_{\tau} m_{\mu}}{m_{H^{ \pm}}^{2}}\left[Z-\frac{f(Z)}{\sqrt{2}} \chi_{33}^{l}\right]\left[Z-\frac{f(Z)}{\sqrt{2}} \chi_{22}^{l}\right] .
$$

One can see that $R$ is symmetric in the two parameters $\chi_{22}$ and $\chi_{33}$. Following the analysis of Ref. [61], we can obtain the following explicit constraint:

$$
\frac{\left|Z_{22} Z_{33}\right|}{m_{H^{ \pm}}^{2}} \leq 0.16 \mathrm{GeV}^{-1} \quad(95 \% \mathrm{CL})
$$

We show in Fig. 1 the constraints on $\chi_{22}^{l}$ and $\chi_{33}^{l}$ with $Z=10,40,80$. One can see that, for small $Z$ values, the allowed region for $\chi_{22}^{l}$ and $\chi_{33}^{l}$ is large whereas, when $Z$ instead grows, the allowed region for theses parameters is smaller in comparison. The constraints becomes most restrictive when $Z$ is large and we have a very light charged Higgs boson, between 90 and $130 \mathrm{GeV}$. The plot also shows that $\chi_{22}^{l}$ and $\chi_{33}^{l}$ could be simultaneously 1 and -1 , respectively, and the more favorable region is the one where $\chi_{22}^{l}=\chi_{33}^{l}=1.5$ for $0.5 \leq Z \leq 100$. When $Z$ is large, if $\chi_{22}^{l}=1$, one can see that $0.5 \leq \chi_{33}^{l} \leq 2.5$ (the same happens when $\chi_{22}^{l}$ and $\chi_{33}^{l}$ are interchanged). Further, in Fig. 2 we present the plane $\left[m_{H^{ \pm}}, X\right]$ and the allowed region is shown for the cases $\chi_{33}^{l}=0$ and $\chi_{22}^{l}=0$ (left panel) and $\chi_{22}^{l}=0.1$ and $-20 \leq \chi_{33}^{l} \leq 20$ (right panel). In the left panel we present the red region (without contributions from the parameters of flavor physics $\left|\chi_{i j}\right|$ ), which is allowed by $\mu-e$ universality in $\tau$ decays: e.g., for $m_{H^{ \pm}} \leq 120 \mathrm{GeV}$ we must have the constraint $X \leq 50$. In the right panel we show two regions: here, the blue(blue\&gray) one is allowed for the cases $5 \leq\left|\chi_{i j}\right|\left(\left|\chi_{i j}\right| \leq 5\right)$. The blue region is clearly the more restrictive one of the two and could become even smaller while the $\left|\chi_{i j}\right|$ 's grow. In the case shown, we can get that $m_{H^{ \pm}} \leq 150 \mathrm{GeV}$ for $X \leq 20$. Conversely, with both regions combined, blue\&gray, 

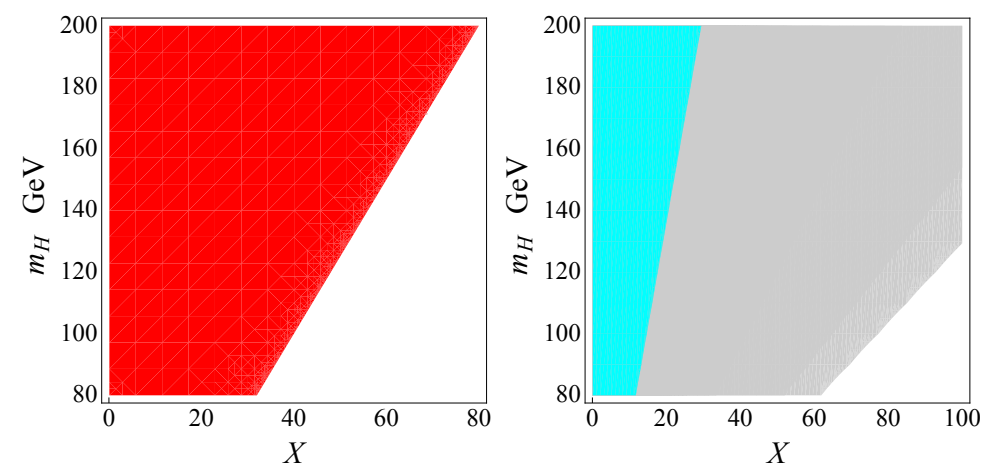

Figure 2. Considering the constraint from $\mu-e$ universality in $\tau$ decays, we show the allowed region for the plane $m_{H^{ \pm}}-X$ : left panel represent the case $\chi_{33}^{l}=0$ and $\chi_{22}^{l}=0$, right panel represents the case $\chi_{22}^{l}=0.1$ and $-20 \leq \chi_{33}^{l} \leq 20$ (blue region is for $5 \leq\left|\chi_{i j}^{l}\right|$, blue\&gray region is for $\left.\left|\chi_{i j}^{l}\right| \leq 5\right)$. The same occur when $\chi_{33}^{l}=0.1$ and $-20 \leq \chi_{22}^{l} \leq 20$.

which represent the portion of parameter space allowed for $0.8 \leq\left|\chi_{i j}\right| \leq 2$, we see that the model is more favored, because it opens up larger regions in the plane $\left[m_{H^{ \pm}}, X\right]$. One can see this, e.g., for $X \leq 80$, as the bound for the charged Higgs boson mass is now given by $m_{H^{ \pm}} \geq 100 \mathrm{GeV}$, that is, not dissimilar from the previous case (when $X \leq 20$ ).

\subsection{Leptonic meson decays}

The leptonic decay of a charged meson, $M \rightarrow l \nu_{l}$, is sensitive to $H^{+}$exchange due to the helicity suppression of the SM amplitude. The total decay width is given by [61, 62]:

$$
\Gamma\left(M_{i j} \rightarrow l \nu\right)=G_{F}^{2} m_{l} f_{M}^{2}\left|V_{i j}\right|^{2} \frac{m_{M_{i j}}}{8 \pi}\left(1+\delta_{e m}\right)\left|1-\Delta_{i j}\right|,
$$

where $i, j$ represent the valence quarks of the meson, $V_{i j}$ is the relevant CKM matrix element, $f_{M}$ is the decay constant of the meson $M$ (the normalization of the meson decay constant correspond to $\left.f_{\pi}=131 \mathrm{MeV}\right), \delta_{e m}$ denotes the electromagnetic radiative contributions and $\Delta_{i j}$ is the correction that comes from new physics information. In particular, for the 2HDM-III employing a four-zero Yukawa texture, the leptonic decays receive a contribution from charged Higgs bosons in the following form:

$$
\Delta_{i j}=\left(\frac{m_{M}}{m_{H^{ \pm}}}\right)^{2} Z_{k k}\left(\frac{Y_{i j} m_{u_{i}}+X_{i j} m_{d_{j}}}{V_{i j}\left(m_{u_{i}}+m_{d_{j}}\right)}\right), \quad k=2,3 .
$$

In the more general 2HDM-III the $\Delta i j$ correction can be a complex number. As is pointed out in [61], in some Two Higgs Doublet Models (2HDM's) with natural flavor conservation the correction $\Delta_{i j}$ (with $\chi^{\prime}$ s $=0$ ) is predicted to be positive (in 2HDM-I) or negative (in 2HDM-X), while can have either sign in 2HDM-II and 2HDM-Y, depending on the decaying meson, whereas it is absent in the inert Higgs scenario.

We focus on decays of heavy pseudoscalar mesons $D \rightarrow \mu \nu, B \rightarrow \tau \nu$ and $D_{s} \rightarrow \mu \nu, \tau \nu$, which have been measured. In $B$ and $D$ decays the function $\Delta_{i j}$, one can neglect the contribution proportional to the light quark mass because $m_{u} / m_{b} \leq m_{d} / m_{c} \sim O\left(10^{-3}\right)$. 
Hence the functions $\Delta_{i j}$ for $D \rightarrow \mu \nu$ and $B \rightarrow \tau \nu$, respectively, are given by:

$$
\begin{aligned}
\Delta_{c d} & \approx \frac{m_{D}^{2}}{m_{H^{ \pm}}^{2}} Z_{22} \frac{Y_{21}}{V_{c d}} \\
& =\frac{m_{D}^{2}}{m_{H^{ \pm}}^{2}}\left(Z-\frac{f(Z)}{\sqrt{2}} \chi_{22}^{l}\right)\left(\left(Y-\frac{f(Y)}{\sqrt{2}} \chi_{22}^{u}\right)-\sqrt{\frac{m_{t}}{m_{c}}} \frac{V_{t d}}{V_{c d}} \frac{f(Y)}{\sqrt{2}} \chi_{23}^{u}\right), \\
\Delta_{u b} & \approx \frac{m_{B}^{2}}{m_{H^{ \pm}}^{2}} Z_{33} \frac{X_{13}}{V_{u b}} \\
& =\frac{m_{B}^{2}}{m_{H^{ \pm}}^{2}}\left(Z-\frac{f(Z)}{\sqrt{2}} \chi_{33}^{l}\right)\left(\left(X-\frac{f(X)}{\sqrt{2}} \chi_{33}^{d}\right)-\sqrt{\frac{m_{s}}{m_{b}}} \frac{V_{u s}}{V_{u b}} \frac{f(X)}{\sqrt{2}} \chi_{23}^{d}\right) .
\end{aligned}
$$

Apparently, the factor $\sqrt{m_{t} / m_{c}}$ in $\Delta_{c d}$ could be considered as a dangerous term, which could make the theoretical predictions deviate from the experimental results, however, the term $V_{t d} / V_{c d}$ reduces this possible effect. Similarly, this happens when one wants to fit the four-zero texture of the Yukawa matrices with the CKM matrix. Since experimental results of $B\left(D^{+} \rightarrow \mu \nu\right)$, which were measured by CLEO collaboration [63], the authors of Ref. [61] found the following constraints at 95\% C.L. for any model: $0.8 \leq\left|1-\Delta_{u b}\right| \leq 2$ and $0.87 \leq\left|1-\Delta_{c d}\right| \leq 1.12$. Considering those constraints, we can get the allowed circular bands in the $Z_{22}^{*} Y_{21} /\left(m_{H^{ \pm}}^{2} V_{c d}\right)$ and $Z_{33}^{*} X_{13} /\left(m_{H^{ \pm}}^{2} V_{u b}\right)$ complex planes. Our numerical analysis obtained from the decays $B \rightarrow \tau \nu$ and $D \rightarrow \mu \nu$ is shown in Fig. 3, which is consistent with the results of [61] when the parameters $\chi^{\prime} s$ are absent. For instance, we also find the real solutions are $Z_{33} X_{13} /\left(m_{H^{ \pm}}^{2} V_{u b}\right) \in[-0.036,0.008] \mathrm{GeV}^{-2}$ or $[0.064,0.108]$ $\mathrm{GeV}^{-2}$ from the $B \rightarrow \tau \nu$, and $Z_{22} Y_{21} /\left(m_{H^{ \pm}}^{2} V_{c d}\right) \in[-0.037,0.035] \mathrm{GeV}^{-2}$ or $[0.535,0.609]$ $\mathrm{GeV}^{-2}$ from the $D \rightarrow \mu \nu$. In Fig. 4 we show the allowed region for the plane $\left[\chi_{22}^{u}, \chi_{23}^{u}\right]$, assuming that $\chi_{22}^{l} \in[0.1,1.5]$, and considering the bounds for $D \rightarrow \mu \nu$. One can see that $\chi_{23}^{u} \in[0.75,1.25]$ when $\chi_{22}^{u}=1$ for $30 \leq|Z|=|Y|$. For the cases $Z>>Y$ or $Y>>Z$ the permitted region is larger than for $1<<|Z|=|Y|$ and $1 \leq \chi_{23}^{u}$. Therefore, $1 \leq \chi_{23}^{u}$ are allowed parameters for the leptonic decay of $D$ mesons and the consequences on the phenomenology of charged Higgs bosons could be an important probe of the flavor structure of the Yukawa sector. Otherwise, for the low energy process $B \rightarrow \tau \nu$ we can get bounds for the parameters of the Yukawa texture pertaining to the $d$-quark family. In Fig. 5, we show the allowed regions in the plane $\left[\chi_{22}^{d}, \chi_{23}^{d}\right]$ for the following cases: $X>>Z$ (left panel), $Z>>X$ (center panel) and $Z, X>>1$ (right panel), with $80 \mathrm{GeV} \leq m_{H^{ \pm}} \leq 160$ $\mathrm{GeV}$ and considering $0.1 \leq \chi_{22}^{l} \leq 1.5$. We can see that the non-diagonal parameter $\chi_{23}^{d}$ is more constrained than $\chi_{23}^{u}$. For the case $Z, X>>1$ and $\chi_{22}^{d}=1$, for $Z=X=20$ we found $\chi_{23}^{d} \in[-0.35,-0.2]$ or $\chi_{23}^{d} \in[0,0.2]$, so that this case could correspond to a 2HDM-II-like scenario (see Tab. 1 ) when $\tan \beta$ is large. This scenario is more constrained when $X=Z \geq 40$ and the bound for $\left|\chi_{23}^{d}\right| \leq 0.2$ is obtained. Another interesting case is when $Z>>X$ and $\chi_{22}^{d}=1$, for $X=0.1$ and $Z=80$ we obtain the following allowed regions: $\chi_{23}^{d} \in[-1.8,-1.2]$ or $\chi_{23}^{d} \in[-0.2,0.6]$, in this scenario it is therefore possible to obtain the constraint $\left|\chi_{23}^{d}\right|=1$. When $X>>Z$ we get a wider permitted region for $\chi_{23}^{d}$, defined in the interval $(-7,2)$.

From $D_{s} \rightarrow \mu \nu, \tau \nu$ decays the constraint $0.97 \leq\left|1-\Delta_{c s}\right| \leq 1.16$ is obtained in Ref. 

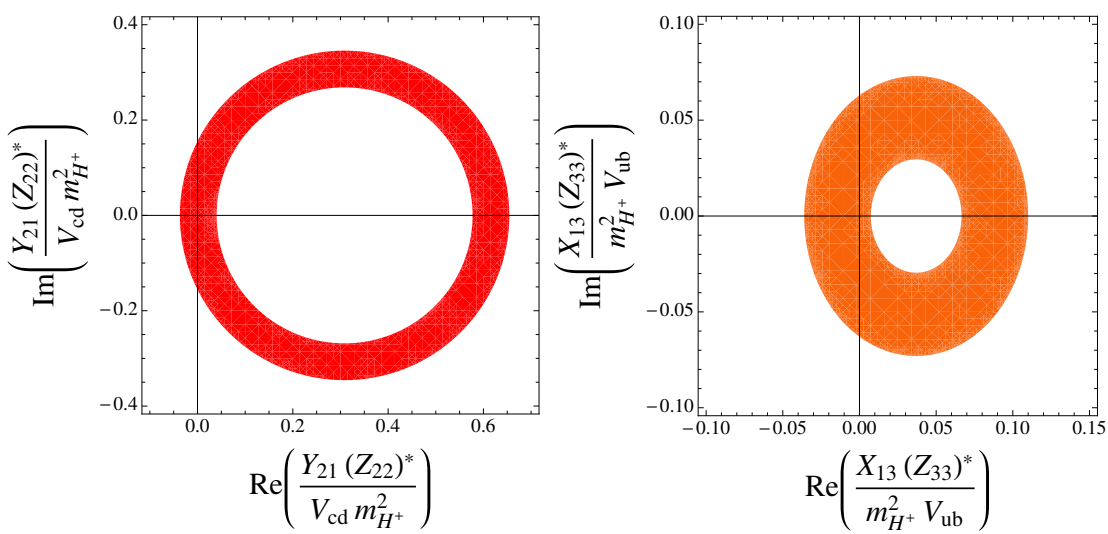

Figure 3. Allowed region for the $Z_{22}^{*} Y_{21} /\left(m_{H^{ \pm}}^{2} V_{c d}\right)$ and $Z_{33}^{*} X_{13} /\left(m_{H^{ \pm}}^{2} V_{u b}\right)$ complex planes from $D \rightarrow \mu \nu$ (left) and $B \rightarrow \tau \nu$ in units of $\mathrm{GeV}^{-2}$.

[61] and one can get the real solutions $\Delta_{c s} / m_{D_{s}}^{2} \in[-0.044,0.008] \mathrm{GeV}^{-2}$ or $[0.545,0.598]$ $\mathrm{GeV}^{-2}$. Here the ratio $m_{s} / m_{c} \approx 10 \%$, thus we cannot neglect $s$-quark effects and the expression for $\Delta_{c s}$ is:

$$
\begin{gathered}
\Delta_{c s}=\left(\frac{m_{D_{s}}}{m_{H^{ \pm}}}\right)^{2} Z_{k k}\left(\frac{Y_{22} m_{c}+X_{22} m_{s}}{V_{c s}\left(m_{c}+m_{s}\right)}\right) \quad(k=2,3), \\
X_{22}=V_{c s}\left(X-\frac{f(X)}{\sqrt{2}} \chi_{22}^{d}\right)-\sqrt{\frac{m b}{m s}} V_{c b} \frac{f(X)}{\sqrt{2}} \chi_{23}^{d}, \\
Y_{22}=V_{c s}\left(Y-\frac{f(Y)}{\sqrt{2}} \chi_{22}^{u}\right)-\sqrt{\frac{m t}{m c}} V_{t s} \frac{f(Y)}{\sqrt{2}} \chi_{23}^{u} .
\end{gathered}
$$

With this information, we can establish a correlation among the parameters that come from $D \rightarrow \mu \nu$ and $B \rightarrow \tau \nu$. Considering the information from $B \rightarrow \tau \nu, D \rightarrow \mu \nu$ and $D_{s} \rightarrow \tau \nu, \mu \nu$, we show in Fig. 6 the constraints for the non-diagonal terms of the Yukawa textures $\chi_{23}^{d}$ and $\chi_{23}^{u}$, assuming $0.1 \leq \chi_{22}^{l}=\chi_{33}^{l} \leq 1.5$, as well as $\chi_{22}^{d}=\chi_{22}^{u}=1$. We present in Tab. 2 a set bounds for these parameters in several scenarios, which are shown in Tab. 1. Combining results from the table, one can derive general constraints for $\left|\chi_{23}^{d}\right| \leq 0.15$ and $\left|\chi_{23}^{u}\right| \leq 1.5$ for almost all scenarios. Only in the 2HDM-Y-like version one can obtain a less stringent bound for $\chi_{23}^{u}$.

\subsection{Semileptonic decays $B \rightarrow D \tau \nu$}

Purely leptonic decays of mesons interwine EW and QCD interactions. However, the role of strong interaction materializes only in the presence of a decay constant, to be assessed through theoretical methods. Semi-leptonic decays are complicated to describe since they involve form factors with a non-trivial dependence on the momentum transfer. If the form factors are known with sufficient accuracy, semi-leptonic BRs start becoming stringent constraints on new physics models. The BaBar and Belle experiments published the first measurements of $B(B \rightarrow D \tau \nu)[64,65]$. Recently, using the full data set collected by 

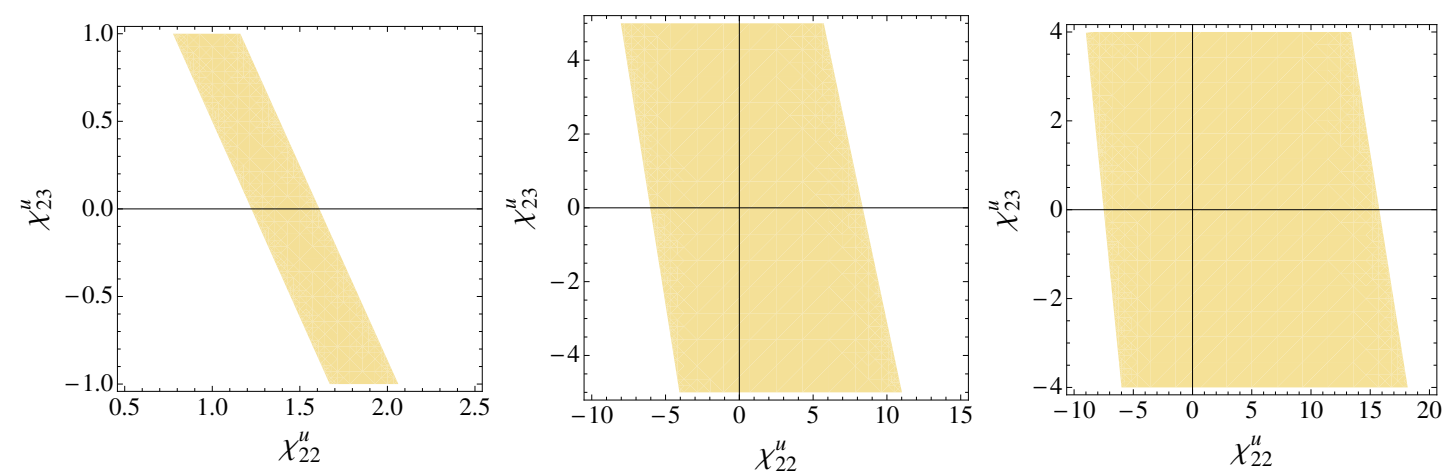

Figure 4. The most constrained region for $\chi_{22}^{u}$ and $\chi_{23}^{u}$ from $D \rightarrow \mu \nu$ for the following cases: $Z, Y>>1$ (left), $Z>>Y$ (center) and $Y>>Z$ (right), with $80 \mathrm{GeV} \leq m_{H^{ \pm}} \leq 160 \mathrm{GeV}$. We assume that $0.1 \leq \chi_{22}^{l} \leq 1.5$.
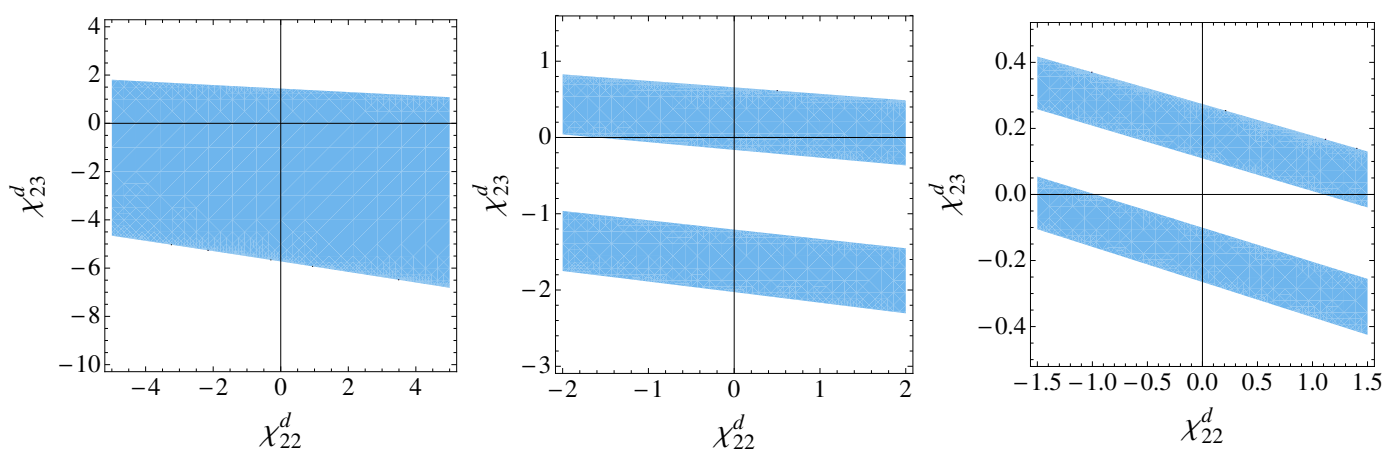

Figure 5. The most constrained region for $\chi_{22}^{d}$ and $\chi_{23}^{d}$ from $B \rightarrow \tau \nu$ for the following cases: $X>>Z$ (left), $Z>>X$ (center) and $Z, X>>1$ (right), with $80 \mathrm{GeV} \leq m_{H^{ \pm}} \leq 160 \mathrm{GeV}$. We assume that $0.1 \leq \chi_{22}^{l} \leq 1.5$.
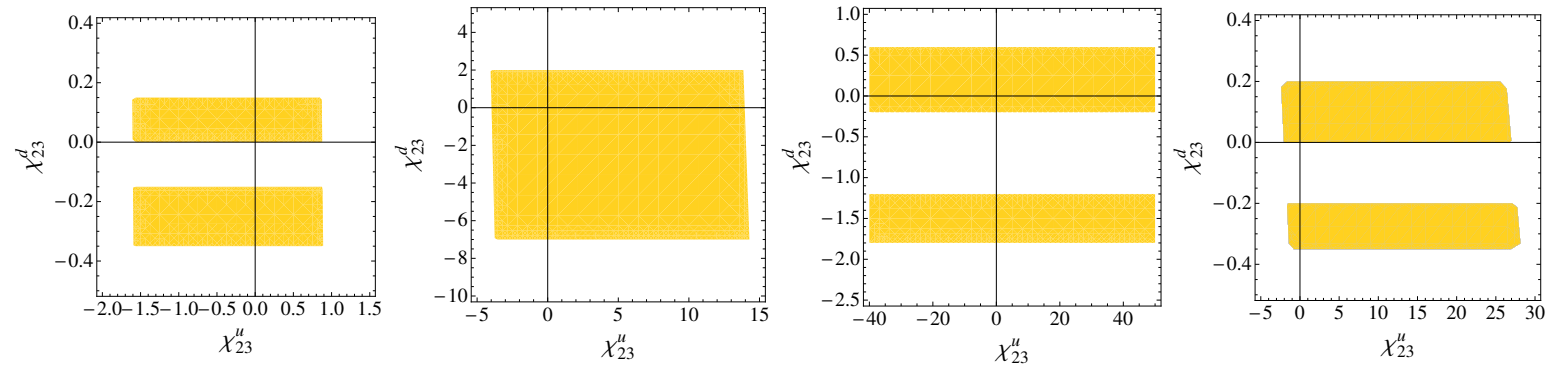

Figure 6. The most constrained region for $\chi_{23}^{d}$ vs. $\chi_{23}^{u}$ from $B \rightarrow \tau \nu, D \rightarrow \mu \nu$ and $D_{s} \rightarrow l \nu$ for the following cases: $|Z|=|X|=|Y|>>1$ (left), $Z>>X, Y$ (center-left), $X>>Y, Z$ (center-right), and $|Z|=|X|>>Y$ (right), with $80 \mathrm{GeV} \leq m_{H^{ \pm}} \leq 160 \mathrm{GeV}$. We assume that $0.1 \leq \chi_{22}^{l} \leq 1.5$ and $\chi_{22}^{u, d}=\chi_{33}^{u, d}=1$.

BaBar, the update of $\mathrm{BR}(B \rightarrow D \tau \nu)$ and $\mathrm{BR}\left(B \rightarrow D^{*} \tau \nu\right)$ was presented in [66], from where it is clear that the 2HDM-II is disfavored. Since this model cannot explain $R(D)$ and $R\left(D^{*}\right)$ simultaneously (and for $B \rightarrow \tau \nu$ a high fine tuning is needed), where $R\left(D^{*}\right)$ 
are the ratios

$$
R\left(D^{*}\right)=\mathrm{BR}\left(B \rightarrow D^{*} \tau \nu\right) / \mathrm{BR}\left(B \rightarrow D^{*} l \nu\right)
$$

with

$$
\begin{aligned}
R(D) & =0.44 \pm 0.058 \pm 0.042 \\
R\left(D^{*}\right) & =0.332 \pm 0.024 \pm 0.018
\end{aligned}
$$

However, lately, in Ref. [67], it was shown that one can simultaneously explain $R(D)$ and $R\left(D^{*}\right)$ in the 2HDM-III with a general flavor structure, where the non-diagonal terms from the $u$-quark sector are relevant.

Following the analysis of Ref. [62], an interesting observable is the normalized BR, $R_{B \rightarrow D \tau \nu}=\operatorname{BR}(B \rightarrow D \tau \nu) / \mathrm{BR}(B \rightarrow D e \nu)$, which corresponds to a $b \rightarrow c$ transition, with a CKM factor much larger than the purely leptonic $B$ decay. One can write this term as a second order polynomial in the charged Higgs boson coupling to fermions, as

$$
R_{B \rightarrow D \tau \nu}=a_{0}+a_{1}\left(m_{B}^{2}-m_{D}^{2}\right) \delta_{23}+a_{2}\left(m_{B}^{2}-m_{D}^{2}\right)^{2} \delta_{23}^{2}
$$

where the factor $\delta_{23}$ is determined by the coupling $H^{+} u_{i} \bar{d}_{i}$, where the general expression for $\delta_{i j}$ is given by

$$
\delta_{i j}=-\frac{Z_{33}}{m_{H^{ \pm}}^{2}}\left(\frac{Y_{i j} m_{u_{i}}-X_{i j} m_{d_{j}}}{m_{u_{i}}-m_{d_{j}}}\right) .
$$

The polynomial coefficients $a_{i}$ in eq. (3.13) are given in Ref. [62] as:

$$
\begin{aligned}
& a_{0}=0.2970+0.1286 d \rho^{2}+0.7379 d \Delta, \\
& a_{1}=0.1065+0.0546 d \rho^{2}+0.4631 d \Delta, \\
& a_{2}=0.0178+0.0010 d \rho^{2}+0.0077 d \Delta,
\end{aligned}
$$

where $d \rho^{2}=\rho^{2}-1.18$ and $d \Delta=\Delta-0.046$ are the variations of the semi-leptonic form factors $\rho^{2}$ and $\Delta[68,69]$. Similarly to the leptonic process $D_{s} \rightarrow l \nu$, we can establish a correlation among the parameters that come from $B \rightarrow D l \nu$ and $B \rightarrow \tau \nu$. In all cases, we consider simultaneously $R(D)$ and $R\left(D^{*}\right)$. One can then constraint the non-diagonal terms of the Yukawa texture, $\chi_{23}^{d}$ and $\chi_{23}^{u}$, by assuming $0.1 \leq \chi_{22}^{l}=\chi_{33}^{l} \leq 1.5$ as well as $\chi_{22}^{d}=\chi_{22}^{u}=1$. We can then show in Tab. 2 a set of bounds for these parameters in several scenarios. By combining results from this table, one can derive general constraints for $\left|\chi_{23}^{d}\right| \leq 0.15$ and $\left|\chi_{23}^{u}\right| \leq 1.5$ for several scenarios (see also Fig. 7). Again, the 2HDMY-like version cannot offer a bound for $\chi_{23}^{u}$ easily. One can see that the 2HDM-III with a Yukawa texture can avoid the constraints of the factor $R_{B \rightarrow D \tau \nu}$ and can thus appear as rather exotic physics, i.e., very different from the traditional $2 \mathrm{HDMs}$ with NFC. In particular, decay channels involving $H^{ \pm} \rightarrow c b$ could be relevant and be searched for in the transition $t \rightarrow H^{ \pm} b[23,41]$, if the $H^{ \pm}$state is sufficiently light. 

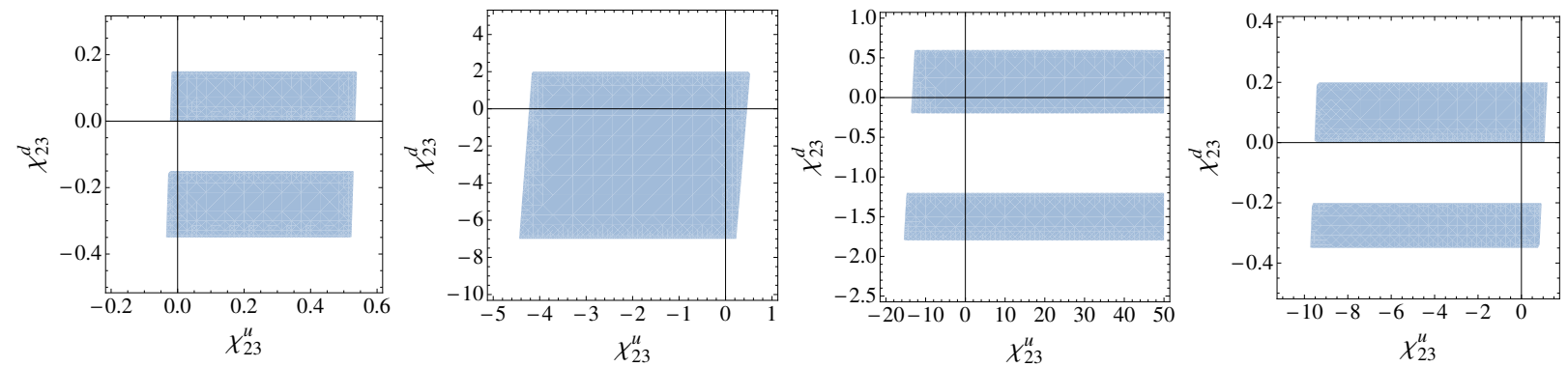

Figure 7. The most constrained region for $\chi_{23}^{d}$ vs. $\chi_{23}^{u}$ from $B \rightarrow \tau \nu$ and $B \rightarrow D \tau \nu$ for the following cases: $|Z|=|X|=|Y|>>1$ (left), $Z>>X, Y$ (center-left), $X>>Y, Z$ (center-right), and $|Z|=|X|>>Y$ (right), with $80 \mathrm{GeV} \leq m_{H^{ \pm}} \leq 160 \mathrm{GeV}$. We assume that $0.1 \leq \chi_{22}^{l} \leq 1.5$ and $\chi_{22}^{u, d}=\chi_{33}^{u, d}=1$.

\begin{tabular}{|c||c|c|c|c|c|}
\hline 2HDM-III's & $\chi_{23}^{d}(B \rightarrow \tau \nu)$ & $\chi_{23}^{u}\left(D_{s} \rightarrow l \nu\right)$ & $\chi_{23}^{u}(B \rightarrow D \tau \nu)$ & $\chi_{23}^{u}$ (combination) & $(X, Y, Z)$ \\
\hline 2HDM-I-like & $\begin{array}{c}(-0.35,-0.15) \text { or } \\
(0,0.15)\end{array}$ & $(-1.5,0.9)$ & $(-0.05,0.45)$ & $(-0.05,0.45)$ & $(20,20,20)$ \\
\hline 2HDM-II-like & $\begin{array}{c}(-0.35,-0.2) \text { or } \\
(0,0.2)\end{array}$ & $(-2,27)$ & $(-9.6,1.2)$ & $(-2,1.2)$ & $(20,0.1,20)$ \\
\hline 2HDM-X-like & $(-7,2)$ & $(-4,14)$ & $(-3.8,0.47)$ & $(-4,0.47)$ & $(0.1,0.1,50)$ \\
\hline 2HDM-Y-like & $\begin{array}{c}(-1.8,-1.2) \text { or } \\
(-0.2,0.6)\end{array}$ & $(-40,50)$ & $(-16,50)$ & $(-16,50)$ & $(50,0.5,0.5)$ \\
\hline
\end{tabular}

Table 2. Constraints from $B \rightarrow D \tau \nu, D_{s} \rightarrow \tau \nu, \mu \nu$ and $B \rightarrow \tau \nu$ decays. We show the allowed intervals for $\chi_{23}^{u, d}$ constrained by each low energy process, according to the different scenarios presented in Tab. 1 as well as the combination of constraints for the $\chi_{23}^{u}$ parameter. We assume $0.1 \leq \chi_{22}^{l}=\chi_{33}^{l} \leq 1.5$ as well as $\chi_{22}^{d}=\chi_{22}^{u}=1$. Taking $80 \mathrm{GeV} \leq m_{H^{ \pm}} \leq 160 \mathrm{GeV}$ and specific values for the $X, Y$ and $Z$ parameters given in Tab. 1 .

\section{4 $B \rightarrow X_{s} \gamma$ decays}

The radiative decay $B \rightarrow X_{s} \gamma$ has been calculated at Next-to-Next-to-Leading Order $(\mathrm{NNLO})$ in the $\mathrm{SM}$, leading to the prediction $\mathrm{BR}\left(B \rightarrow X_{s} \gamma\right)_{\mathrm{SM}}=(3.15 \pm 0.23) \times 10^{-4}[44]$. In the $2 \mathrm{HDM}$ the decay amplitude is known at NLO [43, 70, 71] and in the $2 \mathrm{HDM}$ with Minimal Flavor Violation (MFV) [72] while, only very recently, NNLO results have been presented for both a Type-I and Type-II 2HDM [73]. The current average of the measurements by CLEO [74], Belle [75, 76], and BaBar [77-79] reads $\left.\mathrm{BR}\left(\bar{B} \rightarrow X_{\gamma}\right)\right|_{E_{\gamma}>1.6 \mathrm{GeV}}=$ $(3.37 \pm 0.23) \times 10^{-4}$.

In this subsection we show the constraints on the off-diagonal terms of the four-zero Yukawa texture of the 2HDM-III through a general study of the processes $B \rightarrow X_{s} \gamma$. We first start with a digression on Wilson coefficients entering the higher order calculations. 


\subsubsection{NLO Wilson coefficients at the scale $\mu_{W}$}

To the first order in $\alpha_{s}$, the effective Wilson coefficients at the scale $\mu_{W}=\mathcal{O}\left(M_{W}\right)$ can be written as $[43,80]$

$$
C_{i}^{\mathrm{eff}}\left(\mu_{W}\right)=C_{i}^{0, \mathrm{eff}}\left(\mu_{W}\right)+\frac{\alpha_{s}\left(\mu_{W}\right)}{4 \pi} C_{i}^{1, \mathrm{eff}}\left(\mu_{W}\right) .
$$

The LO contribution of our 2HDM-III version to the relevant Wilson coefficients at the matching energy scale $\mu_{W}$ take the form [43, 80],

$$
\delta C_{(7,8)}^{0, e f f}\left(\mu_{W}\right)=\left|\frac{Y_{33}^{u} Y_{32}^{u *}}{V_{t b} V_{t s}}\right| C_{(7,8), Y Y}^{0}\left(y_{t}\right)+\left|\frac{X_{33}^{u} Y_{32}^{u *}}{V_{t b} V_{t s}}\right| C_{(7,8), X Y}^{0}\left(y_{t}\right)
$$

where $y_{t}=m_{t}^{2} / m_{H^{ \pm}}^{2}, \delta C_{(7,8)}^{0, e f f}\left(\mu_{W}\right)=C_{(7,8)}^{0, e f f}\left(\mu_{W}\right)-C_{(7,8), S M}^{0}\left(\mu_{W}\right)$, and the coefficients $C_{(7,8), S M}^{0,1}\left(\mu_{W}\right), C_{(7,8), Y Y}^{0}\left(y_{t}\right), C_{(7,8), X Y}^{0}\left(y_{t}\right)$ are well known, which are given in $[43,80]$ and

$$
\begin{aligned}
\left|\frac{Y_{33} Y_{32}^{*}}{V_{t b} V_{t s}}\right| & =\left[\left(Y-\frac{f(y)}{\sqrt{2}} \chi_{33}^{u}\right)-\sqrt{\frac{m_{c}}{m_{t}}}\left(\frac{V_{c b}}{V_{t b}}\right) \frac{f(Y)}{\sqrt{2}} \chi_{23}^{u}\right] \\
& \times\left[\left(Y-\frac{f(y)}{\sqrt{2}} \chi_{33}^{u}\right)-\sqrt{\frac{m_{c}}{m_{t}}}\left(\frac{V_{c s}}{V_{t s}}\right) \frac{f(Y)}{\sqrt{2}} \chi_{23}^{u}\right]^{*}, \\
\left|\frac{X_{33} Y_{32}^{*}}{V_{t b} V_{t s}}\right| & =\left[\left(X-\frac{f(X)}{\sqrt{2}} \chi_{33}^{d}\right)-\sqrt{\frac{m_{s}}{m_{b}}}\left(\frac{V_{t s}}{V_{t b}}\right) \frac{f(X)}{\sqrt{2}} \chi_{23}^{d}\right] \\
& \times\left[\left(Y-\frac{f(y)}{\sqrt{2}} \chi_{33}^{u}\right)-\sqrt{\frac{m_{c}}{m_{t}}}\left(\frac{V_{c s}}{V_{t s}}\right) \frac{f(Y)}{\sqrt{2}} \chi_{23}^{u}\right]^{*},
\end{aligned}
$$

The NLO Wilson coefficients at the matching scale $\mu_{W}$ in the 2HDM-III can be written as [43]

$$
\begin{aligned}
C_{1}^{1, \text { eff }}\left(\mu_{W}\right) & =15+6 \ln \frac{\mu_{W}^{2}}{M_{W}^{2}}, \\
C_{4}^{1, \text { eff }}\left(\mu_{W}\right) & =E_{0}+\frac{2}{3} \ln \frac{\mu_{W}^{2}}{M_{W}^{2}}+\left|\frac{Y_{33}^{u} Y_{32}^{u *}}{V_{t b} V_{t s}}\right| E_{H}, \\
C_{i}^{1, \text { eff }}\left(\mu_{W}\right) & =0 \quad(i=2,3,5,6), \\
\delta C_{(7,8)}^{1, \text { eff }}\left(\mu_{W}\right) & =\left|\frac{Y_{33}^{u} Y_{32}^{u *}}{V_{t b} V_{t s}}\right| C_{(7,8), Y Y}^{1}\left(\mu_{W}\right)+\left|\frac{X_{33}^{u} Y_{32}^{u *}}{V_{t b} V_{t s}}\right| C_{(7,8), X Y}^{1}\left(\mu_{W}\right),
\end{aligned}
$$

where the functions on the right-hand side of eqs. (3.21) and (3.23) are given in Ref. $[43,80]$. The contributions of our version 2HDM-III to the $B \rightarrow X_{s} \gamma$ decay are described by the functions $C_{i, j}^{0,1}\left(\mu_{W}\right)(i=7,8$ and $j=(Y Y, X Y))$, as well as the magnitude and sign of the couplings $Y_{33}^{u}, Y_{32}^{u *}$ and $X_{33}^{u}$. Otherwise, in order to compare with previous results, is convenient to write the eqs. (3.18-3.19) as:

$$
\begin{aligned}
\left|\frac{Y_{33} Y_{32}^{*}}{V_{t b} V_{t s}}\right| & =\left[\lambda_{t t}^{U}-\sqrt{\frac{m_{c}}{m_{t}}}\left(\frac{V_{c b}}{V_{t b}}\right) \frac{f(Y)}{\sqrt{2}} \chi_{23}^{u}\right]\left[\lambda_{t t}^{U}-\sqrt{\frac{m_{c}}{m_{t}}}\left(\frac{V_{c s}}{V_{t s}}\right) \frac{f(Y)}{\sqrt{2}} \chi_{23}^{u}\right]^{*}, \\
\left|\frac{X_{33} Y_{32}^{*}}{V_{t b} V_{t s}}\right| & =\left[\lambda_{t t}^{D}-\sqrt{\frac{m_{s}}{m_{b}}}\left(\frac{V_{t s}}{V_{t b}}\right) \frac{f(X)}{\sqrt{2}} \chi_{23}^{d}\right]\left[\lambda_{t t}^{U}-\sqrt{\frac{m_{c}}{m_{t}}}\left(\frac{V_{c s}}{V_{t s}}\right) \frac{f(Y)}{\sqrt{2}} \chi_{23}^{u}\right]^{*},
\end{aligned}
$$


where $\lambda_{t t}^{D}$ and $\lambda_{t t}^{D}$, expressed in eq. (2.40), are parameters defined in a version of the $2 \mathrm{HDM}$-III without off-diagonal terms in the Yukawa texture $[24,80]^{5}$. Again, when the off-diagonal terms of the four-zero texture of Yukawa matrices are absent, we recover the results mentioned. When the heavy charged Higgs bosons is integrated out at the scale $\mu_{W}$, the QCD running of the the Wilson coefficients $C_{i}\left(\mu_{W}\right)$ down to the lower energy scale $\mu_{b}=\mathcal{O}\left(m_{b}\right)$. Thence, for a complete NLO analysis of the radiative decay $B \rightarrow X_{s} \gamma$ only the Wilson coefficient $C_{7}^{e f f}\left(\mu_{b}\right)$ has to be known, which is:

$$
C_{7}^{e f f}\left(\mu_{b}\right)=C_{7}^{0, e f f}\left(\mu_{b}\right)+\frac{\alpha_{s}\left(\mu_{b}\right)}{4 \pi} C_{7}^{1, e f f}\left(\mu_{b}\right)
$$

where the functions $C_{7}^{0, e f f}\left(\mu_{b}\right)$ and $C_{7}^{1, e f f}\left(\mu_{b}\right)$ as functions of $C_{i, j}^{0}\left(\mu_{W}\right)$ and their complete expressions are given in $[43,80]$.

3.5 $\operatorname{BR}\left(B \rightarrow X_{s} \gamma\right)$

The $\mathrm{BR}$ of the inclusive radiative decay $B \rightarrow X_{s} \gamma$ at the LO level is given by [43, 80]:

$$
\mathrm{BR}\left(B \rightarrow X_{s} \gamma\right)_{L O}=B_{S L}\left|\frac{V_{t s}^{*} V_{t b}}{V_{c b}}\right|^{2} \frac{6 \alpha_{e m}}{\pi \theta(z)}\left|C_{7}^{0, e f f}\left(\mu_{b}\right)\right|^{2}
$$

and at the NLO level is

$$
\operatorname{BR}\left(B \rightarrow X_{s} \gamma\right)_{N L O}=B_{S L}\left|\frac{V_{t s}^{*} V_{t b}}{V_{c b}}\right|^{2} \frac{6 \alpha_{e m}}{\pi \theta(z) \kappa(z)}\left[|D|^{2}+A+\Delta\right],
$$

where $B_{S L}=(10.74 \pm 0.16) \%$ is the measured semi-leptonic BR of the $B$ meson [68], $\alpha_{e m}=1 / 137.036$ is the fine-structure constant, $z=m_{c}^{p o l e} / m_{b}^{\text {pole }}$ is the ratio of the quark pole masses, $\theta(z)$ and $\kappa(z)$ denote the phase space factor and the QCD correction [81] for the semi-leptonic $B$ decay and are given in $[43,80]$. The term $D$ in eq. (3.28) corresponds to the sub-processes $b \rightarrow s \gamma[43]$

$$
D=C_{7}^{\mathrm{eff}}\left(\mu_{b}\right)+V\left(\mu_{b}\right)
$$

where the NLO Wilson coefficient $C_{7}^{\text {eff }}\left(\mu_{b}\right)$ has been given in eq. (3.26), and the function $V\left(\mu_{b}\right)$ is given by $[43,80]$. In eq. (3.28), term $A$ is the the correction coming from the bremsstrahlung process $b \rightarrow s \gamma g$ [82]. Now we are ready to present numerical results of the BRs in the 2HDM-III. Following the recent analysis of Refs. [61, 83] and using standard values $[43,80]$ for the charged Higgs boson mass $\left(80 \mathrm{GeV} \leq m_{H^{ \pm}} \leq 300 \mathrm{GeV}\right)$, we can establish the following constraints:

$$
\left|\frac{Y_{33} Y_{32}^{*}}{V_{t b} V_{t s}}\right|<0.25, \quad-1.7<\operatorname{Re}\left[\frac{X_{33} Y_{32}^{*}}{V_{t b} V_{t s}}\right]<0.7 .
$$

Since $\left|\frac{Y_{33} Y_{32}^{*}}{V_{t b} V_{t s}}\right|<0.25$, we show in Fig. 8 the allowed area in the plane $\chi_{33}^{u}-\chi_{23}^{u}$, for the cases $Y<<1$ (left panel), $Y=1$ (center panel) and $Y=10$ (right panel). One can then

\footnotetext{
${ }^{5}$ In the version 2HDM-III of [24,80,84], one has $\lambda_{t t}^{D}=\lambda_{b b}, \lambda_{t t}^{U}=\lambda_{t t}$.
} 

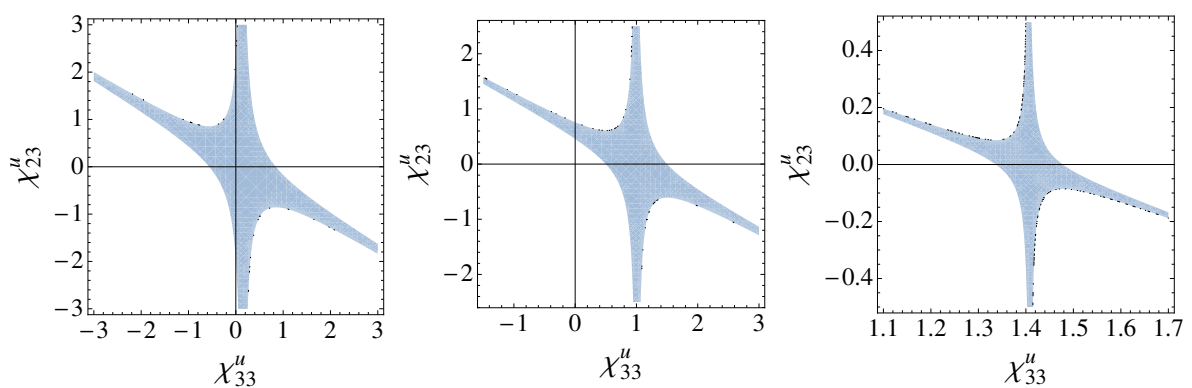

Figure 8. The allowed region for $\chi_{33}^{u}$ vs. $\chi_{23}^{u}$ from $B \rightarrow X_{s} \gamma$ (using the constraint $\left.\left|\frac{Y_{33} Y_{32}^{*}}{V_{t b} V_{t s}}\right|<0.25\right)$ and $B^{0}-\bar{B}^{0}$ mixing (considering the constraint $\left.\left|\frac{Y_{33} Y_{31}^{*}}{V_{t b} V_{t d}}\right|<0.25\right)$ for the following cases: $|Y|<<1$ (left), $Y=1$ (center) and $Y=10$ (right), with $80 \mathrm{GeV} \leq m_{H^{ \pm}} \leq 200 \mathrm{GeV}$.
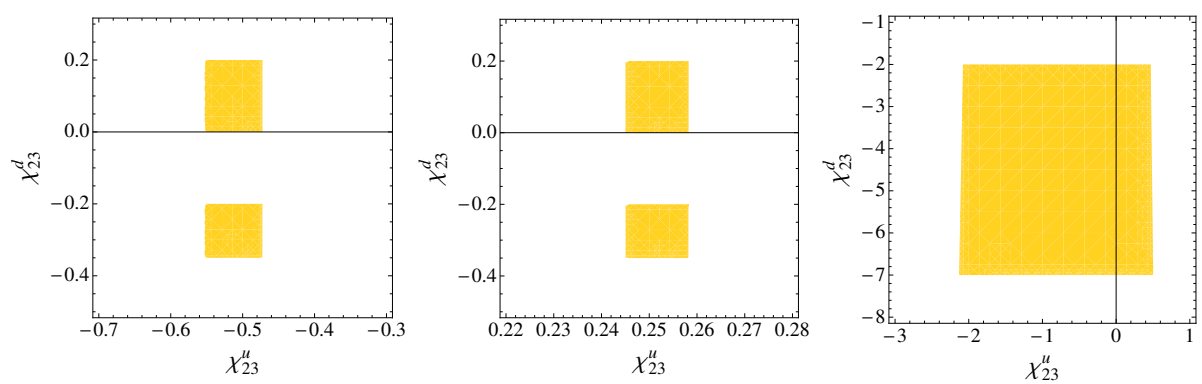

Figure 9. The allowed region for $\chi_{23}^{u}$ vs. $\chi_{23}^{d}$ from $B \rightarrow X_{s} \gamma$ (using the constraint $-1.7<$ $\left.\operatorname{Re}\left[\frac{X_{33} Y_{32}^{*}}{V_{t b} V_{t s}}\right]<0.7\right)$ for the following cases: $X=20$ and $Y=0.1$ (left), $X=Y=20$ (center) and $X=Y=0.1$ (right), with $80 \mathrm{GeV} \leq m_{H^{ \pm}} \leq 200 \mathrm{GeV}$. We assume $\chi_{33}^{u}=1, \chi_{33}^{d}=1$.

extract the bounds $-0.75 \leq \chi_{23}^{u} \leq-0.15$ for $\chi_{33}^{u}=1$ and $0.4 \leq \chi_{23}^{u} \leq 0.9$ for $\chi_{33}^{u}=-1$, both when $Y<<1$. Otherwise, using the second constraint $-1.7<\operatorname{Re}\left[\frac{X_{33} Y_{32}^{*}}{V_{t b} V_{t s}}\right]<0.7$, we can obtain the interval permitted for $\chi_{23}^{u}$, assuming the allowed interval for $\chi_{23}^{d}$ from $B \rightarrow \tau \nu$ and $\chi_{33}^{u}=1=\chi_{33}^{d}=1$. In Fig. 9 one can get the allowed area for some scenarios of Tab. 2. We can, e.g., obtain $\chi_{23}^{u} \in(-0.55,-0.48)$ for the case $X=20$ and $Y=0.1$ (left panel). An interesting scenario for the 2HDM-III is the 2HDM-X-like one, where the allowed region is larger than in other scenarios, with $\chi_{23}^{u} \in(-2.2,0.45)$ and $\chi_{23}^{d} \in(-7,-2)$ (using the constraint coming from $B \rightarrow \tau \nu$ ), so that one can avoid the most restrictive constraints hitherto considered.

\section{6 $\quad B^{0}-\bar{B}^{0}$ mixing}

Remembering that in Ref. [67] the general flavor structure for the 2HDM-III has been found more consistent with various other $B$ physics constraints, we are motivated to test this model assumption also against limits coming from $B^{0}-\bar{B}^{0}$ mixing, where the charged Higgs boson contributes to the mass splitting $\Delta M_{B_{d}}$. Being that $\Delta M_{B_{d}}$ has been measured 
with very high precision [68], we utilize this quantity directly as in Ref. [80, 85] ${ }^{6}$. In the $2 \mathrm{HDMs}$, the NLO mass splitting $\Delta M_{B_{d}}$ is given by $[80,85]$

$$
\Delta M_{B_{d}}=\frac{G_{F}^{2}}{6 \pi^{2}} M_{W}^{2}\left|V_{t d}\right|^{2}\left|V_{t b}\right|^{2} S_{2 H D M}\left(x_{t}, y_{t}\right) \eta_{B}\left(x_{t}, y_{t}\right)\left(B_{B_{d}} f_{B_{d}}^{2} m_{B}\right),
$$

where $x_{t}=\bar{m}_{t}^{2}\left(M_{W}\right) / M_{W}^{2}, y_{t}=\bar{m}_{t}^{2}\left(M_{W}\right) / m_{H^{ \pm}}^{2}$ and

$$
\eta_{B}\left(x_{t}, y_{t}\right)=\alpha_{S}\left(M_{W}\right)^{6 / 23}\left[1+\frac{\alpha_{S}\left(M_{W}\right)}{4 \pi}\left(\frac{D_{2 H D M}\left(x_{t}, y_{t}\right)}{S_{2 H D M}\left(x_{t}, y_{t}\right)}-J_{5}\right)\right]
$$

with

$$
\begin{aligned}
S_{2 H D M}\left(x_{t}, y_{t}\right) & =\left[S_{0}\left(x_{t}\right)+S_{W H}\left(x_{t}, y_{t}\right)+S_{H H}\left(x_{t}, y_{t}\right)\right], \\
D_{2 H D M} & =D_{S M}\left(x_{t}\right)+D_{H}\left(x_{t}, y_{t}\right),
\end{aligned}
$$

here the high energy matching scale $\mu=M_{W}$ is chosen. The functions $D_{S M}\left(x_{t}\right)$ and $D_{H}\left(x_{t}, y_{t}\right)$ of the eq. (3.34) contain the SM and new physics parts of the NLO QCD corrections to the mass difference $\Delta M_{B_{d}}$ [85],

$$
\begin{aligned}
D_{S M}\left(x_{t}\right)= & C_{F}\left[L^{(1, S M)}\left(x_{t}\right)+3 S_{0}\left(x_{t}\right)\right]+C_{A}\left[L^{(8, S M)}\left(x_{t}\right)+5 S_{0}\left(x_{t}\right)\right], \\
D_{H}\left(x_{t}, y_{t}\right)= & C_{F}\left[L^{(1, H)}\left(x_{t}, y_{t}\right)+3\left(S_{W H}\left(x_{t}, y_{t}\right)+S_{H H}\left(x_{t}, y_{t}\right)\right)\right] \\
& +C_{A}\left[L^{(8, H)}\left(x_{t}, y_{t}\right)+5\left(S_{W H}\left(x_{t}, y_{t}\right)+S_{H H}\left(x_{t}, y_{t}\right)\right)\right],
\end{aligned}
$$

where $C_{F}=4 / 3$ and $C_{A}=1 / 3$ for $S U(3)_{C}$. The function $S_{0}\left(x_{t}\right)$ includes the dominant topbox contribution in the SM and has been given in Refs. [80,85]. The functions $S_{W H}\left(x_{t}, y_{t}\right)$ and $S_{H H}\left(x_{t}, y_{t}\right)$ incorporate the new physics contributions from the box diagrams with one or two charged Higgs bosons involved [85],

$$
\begin{aligned}
S_{W H}\left(x_{t}, y_{t}\right)= & \left|\frac{Y_{33}^{*} Y_{31}}{V_{t b} V_{t d}}\right| \frac{y_{t} x_{t}}{4}\left[\frac{\left(2 x_{t}-8 y_{t}\right) \ln \left(y_{t}\right)}{\left(1-y_{t}\right)^{2}\left(y_{t}-x_{t}\right)}+\frac{6 x_{t} \ln \left(x_{t}\right)}{\left(1-x_{t}\right)^{2}\left(y_{t}-x_{t}\right)}\right. \\
& \left.-\frac{8-2 x_{t}}{\left(1-y_{t}\right)\left(1-x_{t}\right)}\right], \\
S_{H H}\left(y_{t}\right)= & \left|\frac{Y_{33}^{*} Y_{31}}{V_{t b} V_{t d}}\right|^{2} \frac{y_{t} x_{t}}{4}\left[\frac{1+y_{t}}{\left(1-y_{t}\right)^{2}}+\frac{2 y_{t} \ln \left[y_{t}\right]}{\left(1-y_{t}\right)^{3}}\right],
\end{aligned}
$$

with

$$
\begin{aligned}
\left|\frac{Y_{33}^{*} Y_{31}}{V_{t b} V_{t d}}\right| & =\left[\left(Y-\frac{f(y)}{\sqrt{2}} \chi_{33}^{u}\right)-\sqrt{\frac{m_{c}}{m_{t}}}\left(\frac{V_{c b}}{V_{t b}}\right) \frac{f(Y)}{\sqrt{2}} \chi_{23}^{u}\right]^{*} \\
& \times\left[\left(Y-\frac{f(y)}{\sqrt{2}} \chi_{33}^{u}\right)-\sqrt{\frac{m_{c}}{m_{t}}}\left(\frac{V_{c d}}{V_{t d}}\right) \frac{f(Y)}{\sqrt{2}} \chi_{23}^{u}\right] \\
& =\left[\lambda_{t t}^{U}-\sqrt{\frac{m_{c}}{m_{t}}}\left(\frac{V_{c b}}{V_{t b}}\right) \frac{f(Y)}{\sqrt{2}} \chi_{23}^{u}\right]^{*}\left[\lambda_{t t}^{U}-\sqrt{\frac{m_{c}}{m_{t}}}\left(\frac{V_{c d}}{V_{t d}}\right) \frac{f(Y)}{\sqrt{2}} \chi_{23}^{u}\right]
\end{aligned}
$$

\footnotetext{
${ }^{6}$ At LO the quantity $x_{d}=\Delta M_{B_{d}} / \Gamma_{B}$ is used [84].
} 
where we have used eq. (2.40), in order to compare with previous results in the literature, where the non-diagonal terms are not considered [24, 80], so that one can recover those results when $\chi_{23}^{u}=0$. Finally, the function $L^{(i, H)}(i=1,8)$ describes the charged Higgs contribution [85]

$$
\begin{aligned}
L^{(i, H)}\left(x_{t}, y_{t}\right) & =2\left|\frac{Y_{33}^{*} Y_{31}}{V_{t b} V_{t d}}\right| W H^{(i)}\left(x_{t}, y_{t}\right)+2\left|\frac{Y_{33}^{*} Y_{31}}{V_{t b} V_{t d}}\right| \Phi H^{(i)}\left(x_{t}, y_{t}\right) \\
& +\left|\frac{Y_{33}^{*} Y_{31}}{V_{t b} V_{t d}}\right|^{2} H H^{(i)}\left(y_{t}\right) .
\end{aligned}
$$

The explicit expressions of the rather complicated functions $W H^{(i)}\left(x_{t}, y_{t}\right), \Phi H^{(i)}\left(x_{t}, y_{t}\right)$ and $H H^{(i)}\left(y_{t}\right)$ can be found in Ref. [85].

Following the analysis of Ref. [80] and considering the areas allowed by the measured $\Delta M_{B_{d}}$ value within a $2 \sigma$ error, when we have a light charged Higgs $\left(80 \mathrm{GeV} \leq m_{H^{ \pm}} \leq 200\right.$ $\mathrm{GeV}$ ) one can extract the limit

$$
\left|\frac{Y_{33}^{*} Y_{31}}{V_{t b} V_{t d}}\right| \leq 0.25
$$

which is consistent with the bounds obtained from $B \rightarrow X_{s} \gamma$ in the previous subsection. We present then Fig. 8, which is the allowed region in the plane $\left[\chi_{33}^{u}, \chi_{23}^{u}\right]$ for the cases $Y<<1$ (left panel), $Y=1$ (center panel) and $Y=10$ (right panel). One can get the bound $-0.75 \leq \chi_{23}^{u} \leq-0.15$ for $\chi_{33}^{u}=1$, and $0.4 \leq \chi_{23}^{u} \leq 0.9$ for $\chi_{33}^{u}=-1$, both when $Y<<1$. This result reduces a lot the intervals of Tab. 2 for the cases presented.

3.7 $Z \rightarrow b \bar{b}$

Other stringent bounds on $\left|\tilde{\chi}_{33}\right|$ come from radiative corrections to the process $Z \rightarrow b \bar{b}$, especially to the $Z$ decay fraction into $b \bar{b}\left(R_{b}\right)$. Following the formulas presented in Refs. $[61,62,72,86], R_{b}$ is parametrized as:

$$
R_{b}=\frac{\Gamma(Z \rightarrow b \bar{b})}{\Gamma(Z \rightarrow \text { hadrons })}=\left(1+\frac{K_{b}}{k_{b}}\right)^{-1}
$$

where

$$
\begin{aligned}
k_{b} & =\left[\left(\bar{g}_{b}^{L}-\bar{g}_{b}^{R}\right)^{2}+\left(\bar{g}_{b}^{L}+\bar{g}_{b}^{R}\right)^{2}\right]\left(1+\frac{3 \alpha}{4 \pi} Q_{q}^{2}\right) \\
K_{b} & =C_{b}^{Q C D} \sum_{q \neq b, t} k_{b}
\end{aligned}
$$

with $C_{b}^{Q C D}=1.0086$, which is a factor that includes QCD corrections, and

$$
\bar{g}_{b}^{L, R}=\bar{g}_{Z b \bar{b}}^{L, R}+\delta \bar{g}^{L, R}
$$

where $g_{Z b \bar{b}}^{L, R}$ are the tree level couplings and $\delta g^{L, R}$ are the radiative corrections that include the contributions of new physics. In models with two doublets $\delta g^{L}$ and $\delta g^{R}$ have contributions from loops involving all the Higgses $\left(H^{ \pm}, H^{0}, h^{0}\right.$ and $\left.A^{0}\right)$. Then, following the 
calculation of Ref. [72], we obtain that the dominant contributions for $\delta g^{L, R}$ come from the charged Higgs boson and are given by

$$
\begin{aligned}
\delta g^{L} & =\frac{\sqrt{2} G_{F} M_{w}^{2}}{16 \pi^{2}}\left[\frac{m_{t}}{M_{w}}\left(Y-\frac{f(Y)}{\sqrt{2}} \chi_{33}^{u}\right)\right]^{2} \\
& \times\left\{\frac{R}{R-1}-\frac{R \log R}{(R-1)^{2}}+f_{2}(R)\right\}, \\
\delta g^{R} & =-\frac{\sqrt{2} G_{F} M_{w}^{2}}{16 \pi^{2}}\left[\frac{m_{b}}{M_{w}}\left(X-\frac{f(X)}{\sqrt{2}} \chi_{33}^{d}\right)\right]^{2} \\
& \times\left\{\frac{R}{R-1}-\frac{R \log R}{(R-1)^{2}}+f_{2}(R)\right\},
\end{aligned}
$$

where $R=m_{t}^{2} / m_{H^{+}}^{2}$ and the function $f_{2}(R)$ governing the NLO corrections is given in Refs. [72] . Again, when $\chi_{33}^{u, d}=0$, one gets the case for the 2HDM-II. According to the measured value of $R_{b}$ [87],

$$
R_{b}=0.21629 \pm 0.00066
$$

we can get the experimental constraints for $\delta R_{b}=|0.00066|$. Then, from eqs. (3.45)-(3.46) we obtain bounds for $\left|\tilde{\chi}_{33}^{f}\right|$ and $X, Y$. From Ref. [61] we can also use the combined limit from leptonic $\tau$ decays and the global fit to (semi-)leptonic decays, which is given by:

$$
\frac{\left|Y_{33} Z_{33}\right|}{m_{H^{ \pm}}^{2}}<0.005, \quad\left|X_{33}\right|<50 .
$$

However, this constraint is already contained in the $b \rightarrow s \gamma$ ones, which are more restrictive, so that this last result does not modify those obtained in the previous section.

\section{$3.8 B_{s} \rightarrow \mu^{+} \mu^{-}$}

A few months ago, the LHCb collaboration found the first evidence for the $B_{s} \rightarrow \mu^{+} \mu^{-}$decay [88], with an experimental value for the $\mathrm{BR}$ given by $\mathrm{BR}\left(B_{s} \rightarrow \mu^{+} \mu^{-}\right)=3.2_{-1.2}^{+1.5} \times 10^{-9}$, which imposes a lower bound on the parameters of our model. Recently, the analysis of Higgs-mediated FCNCs in models with more than one Higgs doublet has been performed [89] and it shows that the MFV case is more stable in suppressing FCNCs than the hypothesis of NFC when the quantum corrections are taken into account ${ }^{7}$. In this work the scalar FCNC interactions have been considered, as it happens in our model. On the other hand, in $[90,91]$, the contribution for the $2 \mathrm{HDM}-\mathrm{II}$ is presented in the regime of large $\tan \beta$, which should be considered in our work in order to get these results when the $\chi_{i j}$ parameters are absent. As was presented in a similar case for another version of the 2HDM-III, where both contributions, at tree and at one-loop level, were taken into account $[92]^{8}$. Then, following the calculation of the BR for the process $B_{s} \rightarrow \mu^{+} \mu^{-}$given in [89, 93, 94], we have

$$
\mathrm{BR}\left(B_{s} \rightarrow \mu^{+} \mu^{-}\right)=\mathrm{BR}\left(B_{s} \rightarrow \mu^{+} \mu^{-}\right) \mathrm{SM}\left(\left|1+R_{p}\right|^{2}+\left|R_{s}\right|^{2}\right)
$$

\footnotetext{
${ }^{7} \mathrm{~A}$ particular case of MVF in the $2 \mathrm{HDM}$ is the A2HDM [89].

${ }^{8}$ The version 2HDM-III presented in [92] is the so-called 2HDM-II-like of our 2HDM-III, where the parameter $\epsilon_{i j}^{f}$ introduced in that reference is related to our parameter $\chi_{i j}^{f}$ in the following way: $\epsilon_{i j}=$ $\frac{\sqrt{m_{i} m_{j}}}{v} \chi_{i j}$.
} 
where $R_{s}$ and $R_{p}$ contain, in the regime $X>>1$, the corrections at one-loop level from charged Higgs bosons and the contribution at tree level of the neutral Higgs bosons which come from the couplings $s \bar{b} \phi$ as well as $b \bar{s} \phi(\phi=H, A)$, as given in (2.31), where the off-diagonal terms $\chi_{23}^{d}$ contribute to $B_{s} \rightarrow \mu^{+} \mu^{-9}$. Therefore, $R_{s}$ and $R_{p}$ are

$$
\begin{aligned}
R_{s}^{\phi s b} & =\frac{4 \pi^{2}}{Y_{0}\left(x_{t}\right) g^{2}}\left(\frac{1}{1+m_{s} / m_{b}}\right)\left(\frac{m_{s}}{m_{b}}\right) \frac{M_{B_{s}}^{2}}{V_{t b} V_{t s}^{*}}\left[\frac{H_{23}^{d} H_{22}^{\ell}}{m_{H^{0}}^{2}}\right], \\
R_{p}^{\phi s b} & =\frac{4 \pi^{2}}{Y_{0}\left(x_{t}\right) g^{2}}\left(\frac{1}{1+m_{s} / m_{b}}\right)\left(\frac{m_{s}}{m_{b}}\right) \frac{M_{B_{s}}^{2}}{V_{t b} V_{t s}^{*}}\left[\frac{A_{23}^{d} A_{22}^{\ell}}{m_{A^{0}}^{2}}\right], \\
R_{s, p}^{\text {loop }} & =\frac{X^{2}}{8 Y_{0}\left(x_{t}\right)}\left(\frac{1}{1+m_{s} / m_{b}}\right) \frac{M_{B_{s}}^{2}}{M_{W}^{2}}\left[\frac{\log (r)}{r-1}\right],
\end{aligned}
$$

with $\phi_{2,3}^{d}$ and $\phi_{22}^{\ell}(\phi=H, A)$ as given in (2.32), $r=m_{H^{ \pm}}^{2} / m t^{2}$, where $m_{b}, m_{s}$ and $m_{t}$ must be evaluated at their matching scales, and

$$
\operatorname{BR}\left(B_{s} \rightarrow \mu^{+} \mu^{-}\right)_{S M}=\frac{G_{F}^{2} \tau_{B_{s}}}{\pi}\left(\frac{g^{2}}{16 \pi^{2}}\right)^{2} M_{B_{s}} F_{B_{s}}^{2} m_{\mu}^{2}\left|V_{t b} V_{t s}^{*}\right|^{2} \sqrt{1-\frac{4 m_{\mu}^{2}}{M_{B_{s}}^{2}}} Y_{0}\left(x_{t}\right)^{2},
$$

here, $Y_{0}\left(x_{t}\right)$ is the loop function given in [93], $M_{B_{s}}$ and $\tau_{B_{s}}$ are the mass and lifetime of the $B_{s}$ meson, respectively, and $F_{B_{s}}=242.0(9.5) \mathrm{MeV}$ is the $B_{s}$ decay constant [95]. Otherwise, from (2.32) and considering some of the cases given in Tab. 1, we can constrain the non-diagonal terms of the Yukawa texture $\chi_{23}^{d}$ and the diagonal terms $\chi_{22}^{\ell}$ as well, for two cases: $X>>Z$ and $X, Z>>1$. We present then Fig. 10 the case $X, Z>>1$, taking $m_{H^{0}}=300 \mathrm{GeV}, 100 \mathrm{GeV} \leq m_{H^{ \pm}} \leq 350 \mathrm{GeV}$ and for the following cases: $m_{A^{0}}=100 \mathrm{GeV}$ (left panel) and $m_{A^{0}}=300 \mathrm{GeV}$ (right panel). One can see that the process $B_{s} \rightarrow \mu^{+} \mu^{-}$ imposes constraints onto the parameter $\chi_{22}^{\ell}$ and $\chi_{23}^{d}$ and that we obtain the following bounds: $-0.1 \leq \chi_{22}^{\ell} \leq 0.4\left(-0.4 \leq \chi_{22}^{\ell} \leq 1\right)$ and $-0.1 \leq \chi_{23}^{d} \leq 0.05\left(-0.4 \leq \chi_{23}^{d} \leq 0.15\right)$ for $m_{A^{0}}=100 \mathrm{GeV}\left(m_{A^{0}}=300 \mathrm{GeV}\right)$. In Fig. 11 we show the allowed region in the plane $\left[\chi_{22}^{\ell}, \chi_{23}^{d}\right]$ for $X>>Z$, and $m_{A^{0}}=100 \mathrm{GeV}$ (left panel) and $m_{A^{0}}=300 \mathrm{GeV}$ (right panel). One can get the bounds $-1.5 \leq \chi_{22}^{\ell} \leq 2\left(-3 \leq \chi_{22}^{\ell} \leq 3\right)$ and $-0.4 \leq \chi_{23}^{d} \leq 0.2$ $\left(-0.5 \leq \chi_{23}^{d} \leq 0.9\right)$ for $m_{A^{0}}=100 \mathrm{GeV}\left(m_{A^{0}}=300 \mathrm{GeV}\right)$. One can see in Figs. 10-11 that, when $\chi_{22}^{\ell}$ is close to 1 , we have that $\chi_{23}^{d} \leq 10^{-2}$. However, when we have for such a parameter that $0.2 \leq \chi_{22}^{\ell} \leq 0.4$, the off-diagonal term in the Yukawa texture, $\chi_{23}^{d}$, can take values in the the interval $[-0.1,0.15]$ for $Z, X>>1$ or $[-0.5,1]$ for $X>>Z$. These results constrain further the parameters $\chi_{i j}^{f}$ and we present in Tab. 3 the combination of these results with the others of the previous sections. We can then conclude from our results that the diagonal term $\chi_{22}^{\ell}$ and the off-diagonal term $\chi_{23}^{d}$ are very sensitive to the process $B_{s} \rightarrow \mu^{+} \mu^{-}$and we observe that $\chi_{23}^{u}$ is also quite sensitive to the process $B \rightarrow X_{s} \gamma$. Besides, the possibility of light charged Higgs bosons is still consistent with the experimental results for the process $B_{s} \rightarrow \mu^{+} \mu^{-}$in our version of the 2HDM-III.

\footnotetext{
${ }^{9}$ For the case $X>>1$, the contribution of the light neutral Higgs boson is neglected because $h_{i j}<<$ $H_{i j} \sim A_{i j} \propto X$, as justified by taking the values of Tab. 1 for some specials cases and $\alpha=\beta-\pi / 2$.
} 

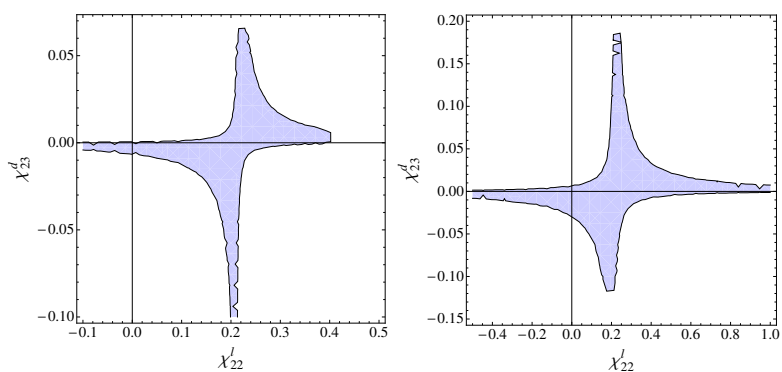

Figure 10. The allowed region for $\chi_{22}^{\chi_{22}^{\prime}}$ vs. $\chi_{23}^{d}$ from $B_{s} \rightarrow \mu^{+} \mu^{-}$, with $X=Z>>1, m_{H^{0}}=300$ $\mathrm{GeV}, 100 \mathrm{GeV} \leq m_{H^{ \pm}} \leq 350 \mathrm{GeV}$, and for the following cases: $m_{A^{0}}=100 \mathrm{GeV}$ (left) and $m_{A^{0}}=300 \mathrm{GeV}$ (rigth).
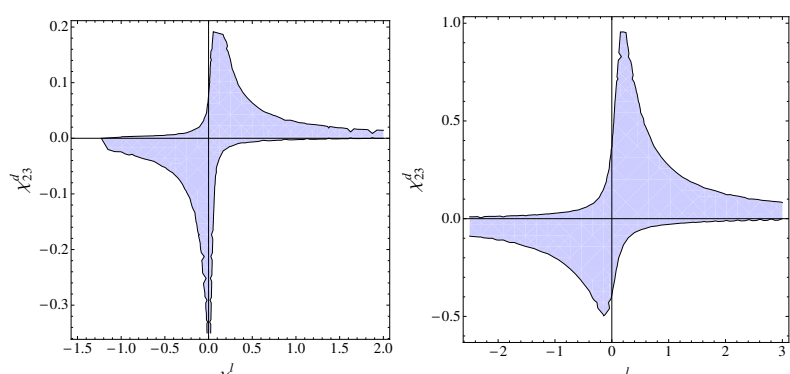

Figure 11. The allowed region for $\chi_{22}^{\chi_{22}^{\prime}}$ vs. $\chi_{23}^{d}$ from $B_{s} \rightarrow \mu^{+} \mu^{-}$, with $X>>Z, m_{H^{0}}=300 \mathrm{GeV}$, $100 \mathrm{GeV} \leq m_{H^{ \pm}} \leq 350 \mathrm{GeV}$, and for the following cases: $m_{A^{0}}=100 \mathrm{GeV}$ (left) and $m_{A^{0}}=300$ $\mathrm{GeV}$ (rigth).

\begin{tabular}{|c||c|c|c|c|}
\hline 2HDM-III's & $\chi_{23}^{d}$ & $\chi_{23}^{u}$ & $\chi_{22}^{\ell}$ & $(X, Y, Z)$ \\
\hline 2HDM-I-like & $(0,0.2)$ & $(0.24,0.26)$ & $(-0.4,1)$ & $(20,20,20)$ \\
\hline 2HDM-II-like & $(0,0.2)$ & $(-0.53,-0.49)$ & $(-0.4,1)$ & $(20,0.1,20)$ \\
\hline 2HDM-X-like & $(-7,2)$ & $(-2.2,0.5)$ & $(-1.5,1.5)$ & $(0.1,0.1,50)$ \\
\hline 2HDM-Y-like & $(-0.2,0.6)$ or & $(-0.53,-0.49)$ & $(-1,1.5)$ & $(50,0.5,0.5)$ \\
\hline
\end{tabular}

Table 3. Constraints from $B \rightarrow D \tau \nu, D_{s} \rightarrow \tau \nu, \mu \nu, B \rightarrow \tau \nu$ and $B_{s} \rightarrow \mu^{+} \mu^{-}$decays. We show the allowed intervals for $\chi_{23}^{u, d}$ and $\chi_{22}^{\ell}$ constrained by each low energy process, according to the different scenarios presented in Tab. 1 as well as the combination of constraints. We assume $0.1 \leq \chi_{33}^{l} \leq 1.5$ as well as $\chi_{22}^{d}=\chi_{22}^{u}=1$. Taking $80 \mathrm{GeV} \leq m_{H^{ \pm}} \leq 350 \mathrm{GeV}$ and specific values for the $X, Y$ and $Z$ parameters given in Tab, 1 .

\section{Light charged Higgs boson phenomenology}

Now we discuss some simple yet interesting phenomenology emerging in the 2HDM-III after all aforementioned constraints are taken into account, in particular the decays of a light charged Higgs boson (i.e., with mass below the top quark one). The expressions for the charged Higgs boson partial decay width $H^{+} \rightarrow u_{i} \bar{d}_{j}$ to massive quark-antiquark pairs 
are of the form

$$
\begin{aligned}
\Gamma\left(H^{+} \rightarrow u_{i} \bar{d}_{j}\right)= & \frac{3 g^{2}}{32 \pi M_{W}^{2} m_{H^{+}}^{3}} \lambda^{1 / 2}\left(m_{H^{+}}^{2}, m_{u_{i}}^{2}, m_{d_{j}}^{2}\right) \\
& \times\left(\frac{1}{2}\left[m_{H^{+}}^{2}-m_{u_{i}}^{2}-m_{d_{j}}^{2}\right]\left(S_{i j}^{2}+P_{i j}^{2}\right)-m_{u_{i}} m_{d_{j}}\left(S_{i j}^{2}-P_{i j}^{2}\right)\right),
\end{aligned}
$$

where $\lambda$ is the usual kinematic factor $\lambda(a, b, c)=(a-b-c)^{2}-4 b c$. When we replace $\tilde{\chi}_{u d} \rightarrow 0$, the formulas of the decay widths become those of the 2HDM with NFC: see, e.g., Refs. [3, 8]. For a scenario with light charged Higgs bosons, for which we can then neglect decays into heavy fermions, the expression for the partial widths of the hadronic decay modes of $H^{ \pm}$'s is reduced to:

$$
\Gamma\left(H^{ \pm} \rightarrow u_{i} \bar{d}_{j}\right)=\frac{3 G_{F} m_{H^{ \pm}}\left(m_{d_{j}}^{2}\left|X_{i j}\right|^{2}+m_{u_{i}}^{2}\left|Y_{i j}\right|^{2}\right)}{4 \pi \sqrt{2}} .
$$

For the case of leptons, one has instead

$$
\Gamma\left(H^{ \pm} \rightarrow l_{j}^{ \pm} \nu_{i}\right)=\frac{G_{F} m_{H^{ \pm}} m_{l_{j}}^{2}\left|Z_{i j}\right|^{2}}{4 \pi \sqrt{2}} .
$$

Again, when the parameters $\chi_{i j}^{f}$ are absent, we recover the results of the MHDM/A2HDM. In $\Gamma\left(H^{ \pm} \rightarrow u_{i} \bar{d}_{j}\right)$ the running quark masses should be evaluated at the scale of $m_{H^{ \pm}}$ and there are QCD vertex corrections which multiply the above partial widths by $(1+$ $\left.17 \alpha_{s} /(3 \pi)\right)$. In the $2 \mathrm{HDM}$ the parameter $\tan \beta$ determines the magnitude of the partial widths. The BRs are well known and for the case of interest, $m_{H^{ \pm}}<m_{t}$, one finds that the dominant decay channel is either $H^{ \pm} \rightarrow c s$ or $H^{ \pm} \rightarrow \tau \nu$, depending on the value of $\tan \beta$. In the $2 \mathrm{HDM}-\mathrm{I}$ the BRs are independent of $\tan \beta$ instead and the $\mathrm{BR}\left(H^{ \pm} \rightarrow \tau \nu\right)$ is about twice the $\operatorname{BR}\left(H^{ \pm} \rightarrow c s\right)$.

The magnitude of $\operatorname{BR}\left(H^{ \pm} \rightarrow c b\right)$ is always less than a few percent in three (Type I, II and lepton-specific) of the four versions of the $2 \mathrm{HDM}$ with NFC, since the decay rate is suppressed by the small CKM matrix element $V_{c b}\left(\ll V_{c s}\right)$. In contrast, a sizeable $\mathrm{BR}\left(H^{ \pm} \rightarrow c b\right)$ can be obtained in the so called 'flipped' $2 \mathrm{HDM}$ [2] for $\tan \beta>3$. This possibility was not stated explicitly in [2] though, where the flipped 2HDM was introduced. The first explicit mention of a large $\operatorname{BR}\left(H^{ \pm} \rightarrow c b\right)$ seems to have been in [8] and a quantitative study followed soon afterwards in [10]. As discussed in [41] though, the condition $m_{H^{ \pm}}<m_{t}$ in the flipped 2HDM would require additional new physics in order to avoid the constraints on $m_{H^{ \pm}}$from $b \rightarrow s \gamma$, while this is not the case in the MHDM/A2HDM. Otherwise, according to the most restrictive flavor constraints obtained in section II, the interaction of charged Higgs bosons with the fermions in the 2HDM-III with a four-zero texture in the Yukawa matrices can be written in the same way as in the MHDM/A2HDM plus an small deviation, namely:

$$
g_{H^{ \pm} u_{i} d_{j}}^{2 \mathrm{HDM}}=g_{H^{ \pm} u_{i} d_{j}}^{\mathrm{MHDM} / \mathrm{AIHDM}}+\Delta g .
$$

Therefore the phenomenology of $H^{ \pm}$states of the 2HDM-III is very close to than in the MHDM/A2HDM, although not necessarily the same. We will show in particular peculiar 
effects induced by the off-diagonal terms of the Yukawa texture studied here in processes considered recently in [41].

\subsection{The dominance of the $\operatorname{BR}\left(H^{ \pm} \rightarrow c b\right)$}

A distinctive signal of a $H^{ \pm}$state from the 2HDM-III for $m_{H^{ \pm}}<m_{t}-m_{b}$ would be a sizable BR for $H^{ \pm} \rightarrow c b$. For $m_{H^{ \pm}}<m_{t}-m_{b}$, the scenario of $|X|>>|Y|,|Z|$ in a 2HDMIII gives rise to a "leptophobic" $H^{ \pm}$with $\mathrm{BR}\left(H^{ \pm} \rightarrow c s\right)+\mathrm{BR}\left(H^{ \pm} \rightarrow c b\right) \sim 100 \%$, as the $\operatorname{BR}\left(H^{ \pm} \rightarrow \tau \nu\right)$ is negligible $(<<1 \%)$. The other decays of $H^{ \pm}$to quarks are subdominant, with the $\mathrm{BR}\left(H^{ \pm} \rightarrow u s\right) \sim 1 \%$ always and the $\operatorname{BR}\left(H^{ \pm} \rightarrow t^{*} b\right)$ becoming sizeable only for $m_{H^{ \pm}} \sim m_{t}$, as can be seen in the numerical analysis in [42] (in the flipped 2HDM). Incidentally, note that the case of $|X|>>|Y|,|Z|$ is obtained in the flipped 2HDM for $\tan \beta>3$, because $|X|=\tan \beta=1 /|Y|=1 /|Z|$ in this scenario.

Conversely, one can see that the configuration $Y>>X, Z$ (this imply that $Y_{i j}>>$ $X_{i j}, Z_{i j}$, see eqs. (2.34)-(2.35)) is very interesting, because the decay $H^{+} \rightarrow c \bar{b}$ is now dominant. In order to show this situation, we calculate the dominant terms $m_{c} Y_{23}, m_{c} Y_{22}$ of the width $\Gamma\left(H^{+} \rightarrow c \bar{b}, c \bar{s}\right)$, respectively, which are given by:

$$
\begin{aligned}
m_{c} Y_{c b} & =m_{c} Y_{23}=V_{c b} m_{c}\left(Y-\frac{f(Y)}{\sqrt{2}} \chi_{22}^{u}\right)-V_{t b} \frac{f(Y)}{\sqrt{2}} \sqrt{m_{t} m_{c}} \chi_{23}^{u} \\
& =V_{c b} m_{c} \lambda_{22}^{u}+V_{t b} \sqrt{m_{t} m_{c}} \lambda_{23}^{u}, \\
m_{c} Y_{c s} & =m_{c} Y_{22}=V_{c s} m_{c}\left(Y-\frac{f(Y)}{\sqrt{2}} \chi_{22}^{u}\right)-V_{t s} \frac{f(Y)}{\sqrt{2}} \sqrt{m_{t} m_{c}} \chi_{23}^{u} \\
& =V_{c s} m_{c} \lambda_{22}^{u}+V_{t s} \sqrt{m_{t} m_{c}} \lambda_{23}^{u} .
\end{aligned}
$$

As $Y$ is large and $f(Y)=\sqrt{1+Y^{2}} \sim Y$, then the term $\left(Y-\frac{f(Y)}{\sqrt{2}} \chi_{22}^{u}\right)$ could be absent or small, when $\chi_{i j}=O(1)$. Besides, the last term is very large because $\propto \sqrt{m_{t} m_{c}}$, given that $m_{t}=173 \mathrm{GeV}$, so that in the end this is the dominant contribution. Therefore, we can compute the ratio of two dominant decays, namely, $\mathrm{BR}\left(H^{ \pm} \rightarrow c b\right)$ and $\operatorname{BR}\left(H^{ \pm} \rightarrow c s\right)$, which is given as follows:

$$
R_{s b}=\frac{\operatorname{BR}\left(H^{ \pm} \rightarrow c b\right)}{\operatorname{BR}\left(H^{ \pm} \rightarrow c s\right)} \sim \frac{\left|V_{t b}\right|^{2}}{\left|V_{t s}\right|^{2}}
$$

In this case $\operatorname{BR}\left(H^{ \pm} \rightarrow c b\right) \sim 100 \%$ (for $m_{H^{ \pm}}<m_{t}-m_{b}$, of course) so that to verify this prediction would really be the hallmark signal of the 2HDM-III. Therefore, we can see that the non-diagonal term $\chi_{23}^{u}$ (or $\lambda_{23}^{u}$ ) cannot be omitted and this is an important result signalling new physics even beyond the standards 2 HDMs. In a similar spirit, one can study other interesting channels, both in decay and production and both at tree and one-loop level [22, 23, 28-30].

Another case is when $X>>Y, Z$, here, we get that the dominants terms are $\propto m_{b} X_{23}$, 
$m_{s} X_{22}$, as

$$
\begin{aligned}
m_{b} X_{c b} & =m_{b} X_{23}=V_{c b} m_{b}\left(X-\frac{f(X)}{\sqrt{2}} \chi_{33}^{d}\right)-V_{c s} \frac{f(X)}{\sqrt{2}} \sqrt{m_{b} m_{s}} \chi_{23}^{d} \\
& =V_{c b} m_{b} \lambda_{33}^{d}+V_{c s} \sqrt{m_{s} m_{b}} \lambda_{23}^{d}, \\
m_{s} X_{c s} & =m_{s} X_{22}=V_{c s} m_{s}\left(X-\frac{f(X)}{\sqrt{2}} \chi_{22}^{d}\right)-V_{t s} \frac{f(X)}{\sqrt{2}} \sqrt{m_{b} m_{s}} \chi_{23}^{d} \\
& =V_{c s} m_{s} \lambda_{22}^{d}+V_{c b} \sqrt{m_{s} m_{b}} \lambda_{32}^{d} .
\end{aligned}
$$

In this case there are two possibilities. If $\chi=O(1)$ and positive then $\left(X-\frac{f(X)}{\sqrt{2}} \chi_{33}^{d}\right)$ is small and

$$
R_{s b} \sim \frac{\left|V_{c s}\right|^{2}}{\left|V_{c b}\right|^{2}}
$$

Here, the $\operatorname{BR}\left(H^{ \pm} \rightarrow c b\right)$ becomes large, again, this case too could be another exotic scenario of the 2HDM-III. The other possibility is when $\chi=O(1)$ and negative, then

$$
R_{s b} \sim \frac{m_{b}^{2}\left|V_{c b}\right|^{2}}{m_{s}^{2}\left|V_{c s}\right|^{2}}
$$

which is very similar to the cases studied recently in [41]. In summary, one can see two possibilities to study the $\operatorname{BR}\left(H^{ \pm} \rightarrow c b\right)$ : firstly, the scenarios given in eqs. (4.7) and (4.10), which are peculiar to the 2HDM-III; secondly, the scenario very close to the MHDM/A2HDM expressed in eq. (4.11).

The CKM matrix elements are well measured, with $V_{c b} \sim 0.04$ (a direct measurement), $V_{c s} \sim 0.97$ (from the assumption that the CKM matrix is unitary), $V_{t s} \sim 0.04$ and $V_{t b} \sim$ 0.999 (from direct determination without assuming unitarity, as is possible from the single top-quark-production cross section) [87]. With this information, it is enough to determine in the cases represented by eqs. (4.7) and (4.10) the dominance of the channel $H^{ \pm} \rightarrow c b$ for light charged Higgs bosons. Otherwise, the situation given in eq. (4.11) is more delicate since the running quark masses $m_{s}$ and $m_{b}$ should be evaluated at the scale $Q=m_{H^{ \pm}}$and this constitutes the main uncertainty in the ratio $R_{b s}$. There is relatively little uncertainty for $m_{b}$, with $m_{b}(Q=100 \mathrm{GeV}) \sim 3 \mathrm{GeV}$. However, there is more uncertainty in the value of $m_{s}$, although in recent years there has been much progress in lattice calculations of $m_{s}$ and an average of six distinct unquenched calculations gives $m_{s}=93.4 \pm 1.1 \mathrm{MeV}[96]$ at the scale of $Q=2 \mathrm{GeV}$. A more conservative average of theses calculations, $m_{s}=94 \pm 3$ $\mathrm{MeV}$, is given in [97]. In [87] the currently preferred range at $Q=2 \mathrm{GeV}$ is given as $80 \mathrm{MeV}<m_{s}<130 \mathrm{MeV}$. Using $m_{s}=93 \mathrm{MeV}$ at the scale $Q=2 \mathrm{GeV}$ (i.e. roughly the central value of the lattice average $[96,97])$ one obtains $m_{s}(Q=100 \mathrm{GeV}) \sim 55 \mathrm{MeV}$. Taking $m_{s}=80 \mathrm{MeV}$ and $m_{s}=130 \mathrm{MeV}$ at $Q=2 \mathrm{GeV}$ one instead obtains $m_{s} \sim 48 \mathrm{MeV}$ and $m_{s} \sim 78 \mathrm{MeV}$, respectively, at $Q=100 \mathrm{GeV}$. Smaller values of $m_{s}$ will give a larger $\mathrm{BR}\left(H^{ \pm} \rightarrow c b\right)$, as can be seen in the situation given in eq. (4.11). Note that the value $m_{s}=55 \mathrm{MeV}$ is significantly smaller than the typical values $m_{s} \sim 150 \rightarrow 200 \mathrm{MeV}$ which were often used in Higgs phenomenology in the past two decades. In essence, we emphasize 
that the scenario $|X|>>|Y|,|Z|$ with $m_{H^{ \pm}}<m_{t}-m_{b}$ has a unique feature in that the magnitude of $m_{s}$ is crucial for determining the relative probability of the two dominant decay channels of the $H^{ \pm}$state. Further, this is not the case for most other non-minimal Higgs sectors with $H^{ \pm}$states that are commonly studied in the literature.

In [10] the magnitude of $\mathrm{BR}\left(H^{ \pm} \rightarrow c b\right)$ in the MHDM was studied in the plane of $|X|$ and $|Y|$, for $|Z|=0$ and 0.5 , taking $m_{s}=0.18 \mathrm{GeV}$ and $m_{b}=5 \mathrm{GeV}$. With these quark masses the maximum value for the above ratio is $R_{b s}=1.23$, which corresponds to $\operatorname{BR}\left(H^{ \pm} \rightarrow c b\right) \sim 55 \%$. However, the values of $m_{s}=0.18 \mathrm{GeV}$ and $m_{b}=5 \mathrm{GeV}$ are no longer realistic (as it was subsequently noted in [40]) and two recent papers $[4,42]$ have in fact updated the magnitude of $R_{b s}$ (in the flipped 2HDM) using realistic running quark masses at the scale $Q=m_{H^{ \pm}}$. In [42], it appears that $m_{s}=0.080 \mathrm{GeV}$ at the scale $Q=m_{H^{ \pm}}$was used, which gives $\operatorname{BR}\left(H^{ \pm} \rightarrow c b\right) \sim 70 \%$, in agreement with the results presented in [41]. In [4], $m_{s}=0.077 \mathrm{GeV}$ at the scale $Q=m_{H^{ \pm}}$was used, with a maximum value for $\operatorname{BR}\left(H^{ \pm} \rightarrow c b\right)$ of $\sim 70 \%$. We note that none of these papers used the precise average $m_{s}=93.4 \pm 1.1 \mathrm{MeV}$ [96] of the lattice calculations, which gives $m_{s} \sim 55 \mathrm{MeV}$ at the scale of $Q=m_{H^{ \pm}}$. This smaller value of $m_{s}$ leads to a maximum value of $\mathrm{BR}\left(H^{ \pm} \rightarrow c b\right)$ which is larger than that given in $[4,10,42]$, as discussed below.

We now study the magnitude of $H^{ \pm} \rightarrow c b$ as a function of the couplings $X, Y, Z$. In Fig. 12 we show the numerical study of $\operatorname{BR}\left(H^{ \pm} \rightarrow c b\right)$ in the plane $[X, Y]$ in the 2HDMIII with $|Z|=0.1$, using $\chi_{i j}^{f}=0.1, m_{s}=0.055 \mathrm{GeV}$ and $m_{b}=2.95 \mathrm{GeV}$ at the scale of $Q=m_{H^{ \pm}}=120 \mathrm{GeV}$. With these values for the quark masses the maximum value is $\mathrm{BR}\left(H^{ \pm} \rightarrow c b\right) \sim 71 \%(91 \%)$ considering $m_{A^{0}}=80 \mathrm{GeV}\left(m_{A^{0}}=100 \mathrm{GeV}\right)$, i.e., a significantly smaller (larger) value than $\mathrm{BR}\left(H^{ \pm} \rightarrow c b\right) \sim 81 \%$ [41], and both larger than $\mathrm{BR}\left(H^{ \pm} \rightarrow c b\right) \sim 55 \%$ in [10]. In Figs. 12 and 13 we also display the bound from $b \rightarrow s \gamma$ (for $\left.m_{H^{ \pm}}=100 \mathrm{GeV}\right)$, which is $\left|\frac{X_{33} Y_{32}^{*}}{V_{t b} V_{t s}}\right|<1.1$ for $\frac{X_{33} Y_{32}^{*}}{V_{t b} V_{t s p}}$ being real and negative plus $\left|\frac{X_{33} Y_{32}^{*}}{V_{t b} V_{t s}}\right|<$ 0.7 for $\frac{X_{33} Y_{32}^{*}}{V_{t b} V_{t s}}$ being real and positive. The parameter space for $\operatorname{BR}\left(H^{ \pm} \rightarrow c b\right)>70 \%(90 \%)$ roughly corresponds to $|X|>10(|X|>5)$ and $|Y|<0.35$ for $\left|\frac{X_{33} Y_{32}^{*}}{V_{t b} V_{t s}}\right|<0.7$. In Fig. 12 and 13 we also show $\operatorname{BR}\left(H^{ \pm} \rightarrow c s\right)$. The latter could be $40 \%(25 \%)$, however, it cannot be maximized for $|Y|>>|X|,|Z|$, because eq. (4.7) does not allow it, whereas $\operatorname{BR}\left(H^{ \pm} \rightarrow \tau \nu\right)$ is maximized for $|Z|>>|X|,|Y|$. In Fig. 14 we show contours of $\operatorname{BR}\left(H^{ \pm} \rightarrow c b\right)$ and $\mathrm{BR}\left(H^{ \pm} \rightarrow \tau \nu\right)$ in the plane $[X, Z]$ for $m_{H^{ \pm}}=120 \mathrm{GeV}, m_{A^{0}}=100 \mathrm{GeV}$ and $|Y|=0.05$. For this value of $|Y|$ the constraint from $b \rightarrow s \gamma$ is always satisfied for the displayed range, $|X|<20$. One can see that the largest values of $\mathrm{BR}\left(H^{ \pm} \rightarrow c b\right)$ arise for $|Z|<2$.

Prospects for $t \rightarrow H^{ \pm} b$ with $H^{ \pm} \rightarrow c b$ at the LHC have been reviewed lately in Ref. [41] for the case of the MHDM/A2HDM, from which many results can however readily be adapted to our current studies. Things go as follow. The case of $m_{H^{ \pm}}<m_{t}-m_{b}$ with a large $\operatorname{BR}\left(H^{ \pm} \rightarrow c s\right)$ can be tested in the decays of the top quark via $t \rightarrow H^{ \pm} b$ and was studied first in [2,98]. Innumerable studies in this direction followed suit, far too many in fact for being listed here. Also, we are concerned here primarily with $H^{ \pm} \rightarrow c b$ decays. The first discussion of $t \rightarrow H^{ \pm} b$ followed by $H^{ \pm} \rightarrow c b$ was given in [39]. Recently, $t \rightarrow H^{ \pm} b$ with $H^{ \pm} \rightarrow c b$ has been studied in the context of the flipped 2HDM [42] and the 2HDM 

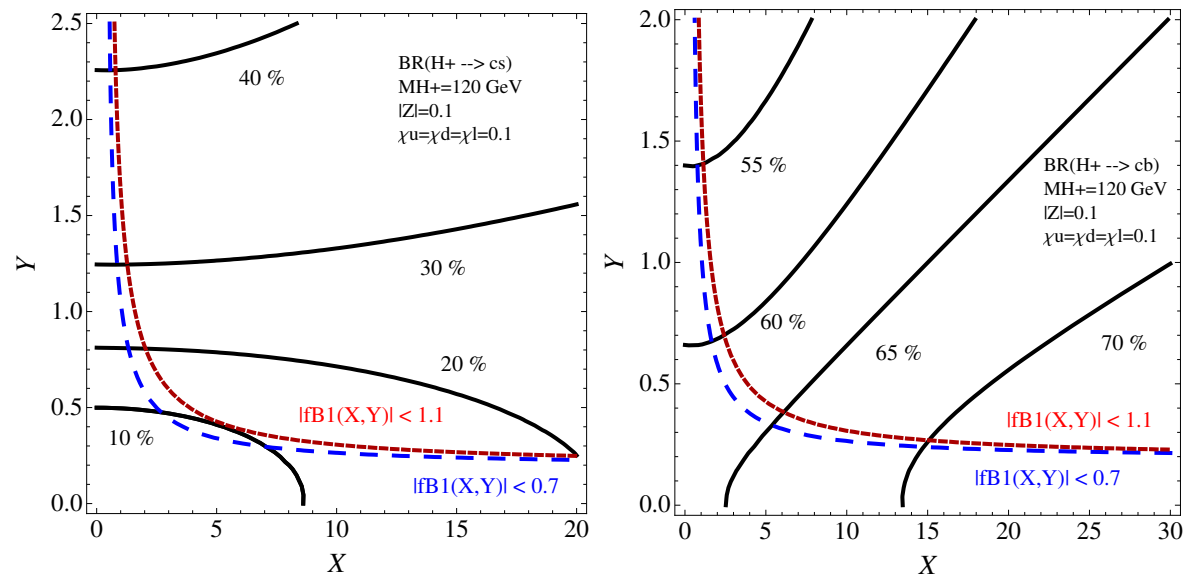

Figure 12. For the exotic scenario of eq. (4.10) we show the contours of the $\operatorname{BR}\left(H^{ \pm} \rightarrow c s\right)$ (left) and $\operatorname{BR}\left(H^{ \pm} \rightarrow c b\right)$ (right) in the plane $[X, Y]$ with $Z=0.1, m_{A^{0}}=80 \mathrm{GeV}, m_{H^{ \pm}}=120 \mathrm{GeV}$ and $\chi_{i j}^{f}=0.1$. The constraint $b \rightarrow s \gamma$ is shown as $|f B 1(X, Y)|<1.1$ for $\operatorname{Re}[f B 1(X, Y)]<0$, where $f B 1(X, Y)=\frac{X_{33} Y_{32}^{*}}{V_{t b} V_{t s}}$ is given in eq. (3.25). For $|f B 1(X, Y)|<0.7$, it is when $\operatorname{Re}[f B 1(X, Y)]<0$. We take $m_{s}\left(Q=m_{H^{ \pm}}\right)=0.055 \mathrm{GeV}$.
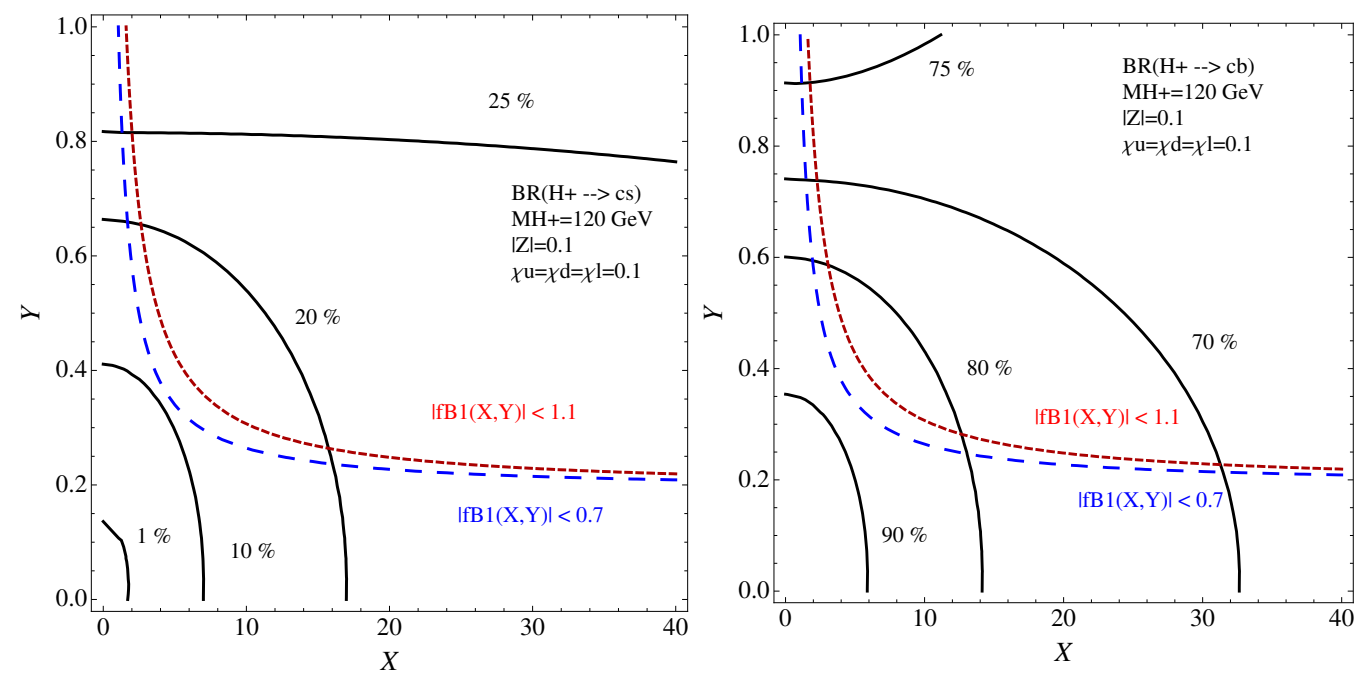

Figure 13. The same as Fig. 12 but for $m_{A^{0}}=100 \mathrm{GeV}$.

without NFC [23] (other than in [41]).

Light charged Higgs bosons $\left(H^{ \pm}\right)$are being searched for (or simulated) in the decays of top quarks $\left(t \rightarrow H^{ \pm} b\right)$ at the Tevatron and at the LHC in a variety of modes [34$36,99,100]$. In particular, separate searches are being carried out for the decay channels $H^{ \pm} \rightarrow c s$ and $H^{ \pm} \rightarrow \tau \nu$, with comparable sensitivity to the mass and fermionic couplings of $H^{ \pm}$. The searches for $H^{ \pm} \rightarrow c s$ in [35] and [36] look for a peak at $m_{H^{ \pm}}$in the dijet invariant mass distribution, with the assumption that neither of the quarks is a $b$-quark.

In the MHDM/A2HDM realisations the $\mathrm{BR}\left(H^{ \pm} \rightarrow c b\right)$ can be as large as $80 \%$ for an $H^{ \pm}$that is light enough to be generated in the $t \rightarrow H^{ \pm} b$ decay. This should be 

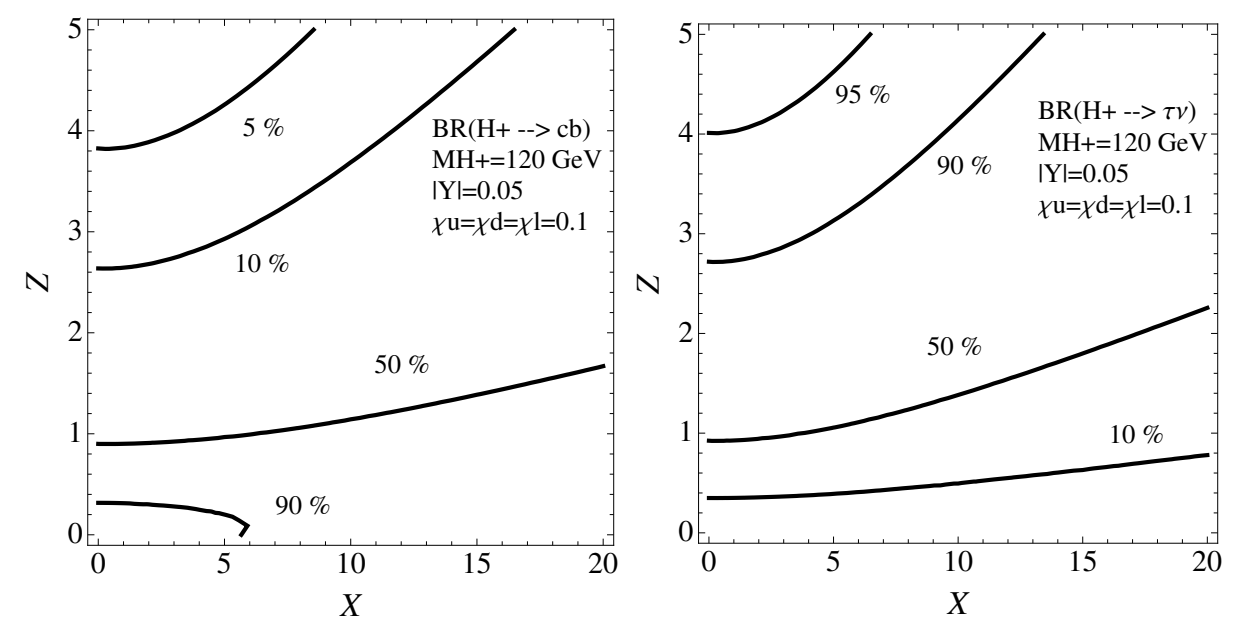

Figure 14. For the exotic scenario of the eq. (4.7), we show the contours of the $\operatorname{BR}\left(H^{ \pm} \rightarrow c b\right)$ (left) and $\operatorname{BR}\left(H^{ \pm} \rightarrow \tau \nu\right)$ (right) in the plane $[X, Z]$ with $Z=0.05, Z>>X, m_{H^{ \pm}}=120 \mathrm{GeV}$ and $\chi_{i j}^{f}=0.1$. We take $m_{s}\left(Q=m_{H^{ \pm}}\right)=0.055 \mathrm{GeV}$.

contrasted to the case of the $H^{ \pm}$state belonging to more standard 2HDMs for which a large $\mathrm{BR}\left(H^{ \pm} \rightarrow c b\right)$ is certainly possible but one expects $m_{H^{ \pm}}>m_{t}$ in order to comply with the measured value of $b \rightarrow s \gamma$, which is not the case for the 2HDM-III. The latter scenario is therefore on the same footing as the MHDM/A2HDM cases studied in [41]. Herein, in short, it was suggested that a dedicated search for $t \rightarrow H^{ \pm} b$ and $H^{ \pm} \rightarrow c b$ would probe values of the fermionic couplings of $H^{ \pm}$which are currently not excluded if one required a $b$-tag in one of the jets originating from $H^{ \pm}$, thus affording in turn sensitivity to smaller values of $\mathrm{BR}\left(t \rightarrow H^{ \pm} b\right)$ than those obtained to date (which use un-flavored jet samples). Therefore, a dedicated search for $t \rightarrow H^{ \pm} b$ and $H^{ \pm} \rightarrow c b$ at the Tevatron and LHC would be a well-motivated and at the same time simple extension of ongoing searches for $t \rightarrow H^{ \pm} b$ with decay $H^{ \pm} \rightarrow c s$.

\subsection{The decay $H^{ \pm} \rightarrow A W^{*}$ for $m_{A^{0}}<m_{H^{ \pm}}$}

The above discussion has assumed that $H^{ \pm}$cannot decay into other (pseudo)scalars. We now briefly discuss the impact of the decay channel $H^{ \pm} \rightarrow A^{0} W^{*}$, which has been studied in the 2HDM-II in [101] and in other 2HDMs with small $|X|,|Y|$ and $|Z|$ in [40], in the light of direct searches at LEP (assuming $A^{0} \rightarrow b \bar{b}$ ) performed in [102]. In a general non-SUSY $2 \mathrm{HDM}$ the masses of the scalars can be taken as free parameters. This is in contrast to the MSSM in which one expects $m_{H^{ \pm}} \sim m_{A^{0}}$ in most of the parameter space. The scenarios of $m_{A^{0}}<m_{H^{ \pm}}$and $m_{A^{0}}>m_{H^{ \pm}}$are both possible in a 2HDM, but large mass splittings among the scalars lead to sizeable contributions to EW precision observables [103], which are parametrized by, e.g., the $S, T$ and $U$ parameters [104]. The case of exact degeneracy $\left(m_{A^{0}}=m_{H^{0}}=m_{H^{ \pm}}\right)$leads to values of $S, T$ and $U$ which are almost identical to those of the SM. A recent analysis in a generic $2 \mathrm{HDM}[105]$ sets $m_{H^{0}}=m_{A^{0}}, \sin (\beta-\alpha)=1$ and studies the maximum value of the mass splitting $\Delta m=m_{A^{0}}-m_{H^{ \pm}}$(for earlier studies see [106]). For $m_{A^{0}}=100 \mathrm{GeV}$ the range $-70 \mathrm{GeV}<\Delta m<20 \mathrm{GeV}$ is allowed, 
which corresponds to $80 \mathrm{GeV}<m_{H^{ \pm}}<170 \mathrm{GeV}$. For $m_{A^{0}}=150 \mathrm{GeV}$ the allowed range is instead $-70 \mathrm{GeV}<\Delta m<70 \mathrm{GeV}$, which corresponds to $80 \mathrm{GeV}<m_{H^{ \pm}}<220 \mathrm{GeV}$. Consequently, sizeable mass splittings (of either sign) of the scalars are possible. Analogous studies in a MHDM have been performed in [107], with similar conclusions.

If $m_{A^{0}}<m_{H^{ \pm}}$then the decay channel $H^{ \pm} \rightarrow A^{0} W^{*}$ can compete with the above decays of $H^{ \pm}$to fermions, because the coupling $H^{ \pm} A^{0} W$ is not suppressed by any small parameter. In Fig. 15 (left) we show contours of $\mathrm{BR}\left(H^{ \pm} \rightarrow A^{0} W^{*}\right)$ in the plane $[X, Y]$ with $|Z|=0.1, m_{A^{0}}=125 \mathrm{GeV}$ and $m_{H^{ \pm}}=150 \mathrm{GeV}$ whereas in Fig. 15 (right) we present the case $m_{A^{0}}=80 \mathrm{GeV}$ and $m_{H^{ \pm}}=120 \mathrm{GeV}$. The contours are presented in the parameter space of interest (i.e., $|Y|<1$ and $|X|>>1$ ) because the contribution of the term $m_{c}^{2}\left|Y_{22}\right|^{2}$ to the decay widths of $H^{ \pm}$to fermions could decrease. Comparing, e.g., Fig. 15 (right) to Fig. 12 (right) one can see that for $|X| \sim 4$ a $\mathrm{BR}\left(H^{ \pm} \rightarrow A^{0} W^{*}\right) \sim 30 \%$ is competitive with a $\operatorname{BR}\left(H^{ \pm} \rightarrow c b\right) \sim 65 \%$. For smaller $m_{A^{0}}$ (e.g., less than $80 \mathrm{GeV}$ ) the contour of the $\operatorname{BR}\left(H^{ \pm} \rightarrow A^{0} W^{*}\right)=50 \%$ would move to higher values of $|X|$. Since the dominant decay of the $A^{0}$ is expected to be $A^{0} \rightarrow b \bar{b}$, the detection prospects in this channel should also be promising because there would be more $b$ quarks from $t \rightarrow H^{ \pm} b, H^{ \pm} \rightarrow A^{0} W^{*}, A^{0} \rightarrow b \bar{b}$ than from $t \rightarrow H^{ \pm} b$ with $H^{ \pm} \rightarrow c b$. We note that there has been a search by the Tevatron for the channel $t \rightarrow H^{ \pm} b, H^{ \pm} \rightarrow A^{0} W^{*}, A^{0} \rightarrow \tau^{+} \tau^{-}$[108], for the case of $m_{A^{0}}<2 m_{b}$ where $A^{0} \rightarrow b \bar{b}$ is not possible [109].

At present there is much speculation about an excess of events around a mass of 125 $\mathrm{GeV}$ in the search for the SM Higgs boson [46]. An interpretation of these events as originating from the process $g g \rightarrow A^{0} \rightarrow \gamma \gamma$ has been suggested in [110]. In Fig. 15 (right) we set $m_{A^{0}}=125 \mathrm{GeV}$ and $m_{H^{ \pm}}=150 \mathrm{GeV}$ for this reason. Since the mass splitting between $H^{ \pm}$and $A^{0}$ is less than in Fig. 15 (left), the contours move to lower values of $|X|$, but a $\operatorname{BR}\left(H^{ \pm} \rightarrow A^{0} W^{*}\right)=4 \%$ is still possible for $|X|<2$. We note that if the excess of events at $125 \mathrm{GeV}$ is attributed to a SM-like Higgs, then in the context of a 2HDM, a candidate would be the lightest CP-even Higgs $h^{0}$ with a coupling to vector bosons of SM strength (recent studies of this possibility can be found in [111]). This scenario would correspond to $\sin (\beta-\alpha) \sim 1$ in a $2 \mathrm{HDM}$ and therefore the coupling $H^{ \pm} h^{0} W$ (with a magnitude $\sim \cos (\beta-\alpha)$ in a $2 \mathrm{HDM})$ would be close to zero. Hence the decay $H^{ \pm} \rightarrow h^{0} W^{*}$ would be suppressed by this small coupling, as well as by the high virtuality of $W^{*}$. Several recent studies $[112,113]$ fit the current data in all the Higgs search channels to the case of a neutral Higgs boson with arbitrary couplings. A SM-like Higgs boson gives a good fit to the data, although a slight preference for non-SM like couplings is emphasized in [113]. If the excess of events at $125 \mathrm{GeV}$ turns out to be genuine and is well described by a non-SM like Higgs boson of a 2 HDM-III with a value of $\sin (\beta-\alpha)$ which is significantly less than unity, then the $\operatorname{BR}\left(H^{ \pm} \rightarrow h^{0} W^{*}\right)$ could be sizeable, with a magnitude given by Fig. 15 (right) after scaling by $\cos ^{2}(\beta-\alpha)$. 

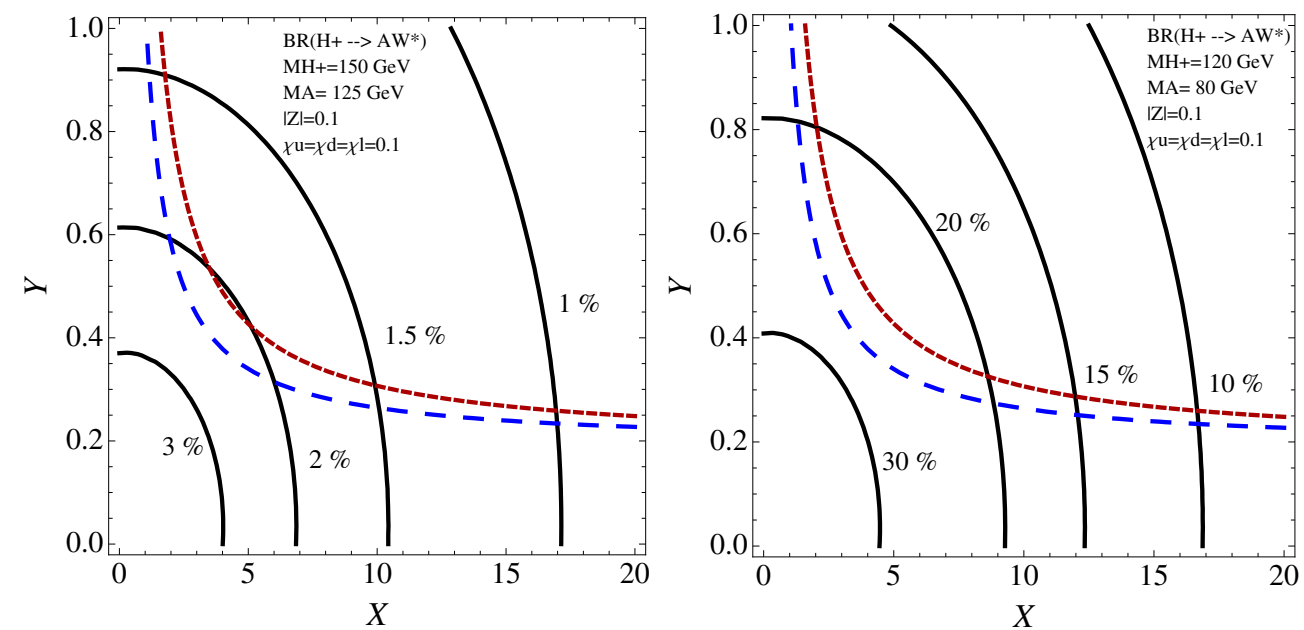

Figure 15. We show the contours of the $\mathrm{BR}\left(H^{ \pm} \rightarrow A W^{*}\right)$ for $m_{H^{ \pm}}=150 \mathrm{GeV}, m_{A^{0}}=125$ $\mathrm{GeV}$ (left) and $m_{H^{ \pm}}=120 \mathrm{GeV}, m_{A^{0}}=80 \mathrm{GeV}$ (right) in the plane $[X, Y]$ with $Z=0.1$, $X>>Y$ and $\chi_{i j}^{f}=0.1$ (all positive). The constraint $b \rightarrow s \gamma$ is shown as $|f B 1(X, Y)|<1.1$ for $\operatorname{Re}[f B 1(X, Y)]<0$ (red-dashed), and $|f B 1(X, Y)|<0.7$ is when $\operatorname{Re}[f B 1(X, Y)]<0$ (blue-dashed). We take $m_{s}\left(Q=m_{H^{ \pm}}\right)=0.055 \mathrm{GeV}$.

\section{$5 \quad$ Numerical Results}

\subsection{Indirect $H^{ \pm}$production}

We now quantify the magnitude of $H^{ \pm} \rightarrow c b$ events produced in the decays of $t$ quarks, and compare this with the expected sensitivity at the LHC. For the partial decay widths of $t \rightarrow W^{ \pm} b$ and $t \rightarrow H^{ \pm} b$ we use the LO expressions (with $\left|V_{t b}\right|=1$ ) as follows:

$$
\begin{array}{r}
\Gamma\left(t \rightarrow W^{ \pm} b\right)=\frac{G_{F} m_{t}}{8 \sqrt{2} \pi}\left[m_{t}^{2}+2 M_{W}^{2}\right]\left[1-M_{W}^{2} / m_{t}^{2}\right]^{2} \\
\Gamma\left(t \rightarrow H^{ \pm} b\right)=\frac{G_{F} m_{t}}{8 \sqrt{2} \pi}\left[m_{t}^{2}\left|Y_{33}\right|^{2}+m_{b}^{2}\left|X_{33}\right|^{2}\right]\left[1-m_{H^{ \pm}}^{2} / m_{t}^{2}\right]^{2} .
\end{array}
$$

The multiplicative (vertex) QCD corrections to both $t \rightarrow W^{ \pm} b$ and $t \rightarrow H^{ \pm} b$ essentially cancel out in the ratio of partial widths [114]. In the phase-space function of both decays we neglect $m_{b}$, and in the terms $m_{t}^{2}\left|Y_{33}\right|^{2}$ and $m_{b}^{2}\left|X_{33}\right|^{2}$ we use $m_{t}=175 \mathrm{GeV}$ and $m_{b}$ evaluated at the scale $m_{H^{ \pm}}$(i.e., $m_{b} \sim 2.95 \mathrm{GeV}$ ).

In Fig. 16 we show contours of the sum of

$$
\mathrm{BR}\left(t \rightarrow H^{ \pm} b\right) \times\left[\mathrm{BR}\left(H^{ \pm} \rightarrow c s\right)+\mathrm{BR}\left(H^{ \pm} \rightarrow c b\right)\right]
$$

in the plane of $[X, Y]$ for $m_{H^{ \pm}}=120 \mathrm{GeV}$ and $m_{H^{ \pm}}=80 \mathrm{GeV}$, setting $|Z|=0.1$. The cross section in eq. (5.3) is the signature to which the current search strategy at the Tevatron and the LHC is sensitive, i.e., one $b$-tag (LHC [36]) or two $b$-tags (Tevatron [35]) are applied to the jets originating from the $t \bar{t}$ decay, but no $b$-tag is applied to the jets originating from $H^{ \pm}$. For the case of $\left[\mathrm{BR}\left(H^{ \pm} \rightarrow c s\right)+\mathrm{BR}\left(H^{ \pm} \rightarrow c b\right)\right]=100 \%$ the current experimental limits for $m_{H^{ \pm}}=120 \mathrm{GeV}$ are $\mathrm{BR}\left(t \rightarrow H^{ \pm} b\right)<0.14$ from ATLAS with $0.035 \mathrm{fb}^{-1}$ [36], 
$\mathrm{BR}\left(t \rightarrow H^{ \pm} b\right)<0.12$ from $\mathrm{CDF}$ with $2.2 \mathrm{fb}^{-1}[35]$, and $\mathrm{BR}\left(t \rightarrow H^{ \pm} b\right)<0.22$ from D0 with $1 \mathrm{fb}^{-1}$ [34]. In Fig. 16 (left) for $m_{H^{ \pm}}=120 \mathrm{GeV}$ these upper limits would exclude the parameter space of $|X|>50$ and small $|Y|$ which is not excluded by the constraint from $b \rightarrow s \gamma$. For the mass region $80 \mathrm{GeV}<m_{H^{ \pm}}<90 \mathrm{GeV}$ there is only a limit from the D0 search in [34], which gives $\operatorname{BR}\left(t \rightarrow H^{ \pm} b\right)<0.21$. From Fig. 16 (right), for $m_{H^{ \pm}}=80 \mathrm{GeV}$, one can see that this limit excludes the parameter space with $|X|>45$ and small $|Y|$.

In Fig. 16 we show contours of $1 \%$, which might be reachable in the $8 \mathrm{TeV}$ run of the LHC. Simulations by ATLAS (with $\sqrt{s}=7 \mathrm{TeV}$ ) for $H^{ \pm} \rightarrow c s$ [100] have shown that the LHC should be able to probe values of $\mathrm{BR}\left(t \rightarrow H^{ \pm} b\right)>0.05$ with $1 \mathrm{fb}^{-1}$ for $m_{H^{ \pm}}>110$ $\mathrm{GeV}$, with the greatest sensitivity being around $m_{H^{ \pm}}=130 \mathrm{GeV}$. For the operation with $\sqrt{s}=8 \mathrm{TeV}$ and an anticipated integrated luminosity of $15 \mathrm{fb}^{-1}$ one expects increased sensitivity (e.g. $\mathrm{BR}\left(t \rightarrow H^{ \pm} b\right)>0.01$ for $m_{H^{ \pm}}>110 \mathrm{GeV}$ ), although the region $80 \mathrm{GeV}<$ $m_{H^{ \pm}}<90 \mathrm{GeV}$ might remain difficult to probe with the strategy of reconstructing the jets from $H^{ \pm}$. An alternative way to probe the region $80 \mathrm{GeV}<m_{H^{ \pm}}<90 \mathrm{GeV}$ is to use the search strategy by D0 in [34], and presumably the LHC could improve on the Tevatron limit on $\operatorname{BR}\left(t \rightarrow H^{ \pm} b\right)<0.21$ for this narrow mass region. From Fig. 16 (left) (for $m_{H^{ \pm}}=120$ $\mathrm{GeV}$ ) one can see that the region of $|Y|>0.32$ and $|X|<14$, which is not excluded by $b \rightarrow s \gamma$, would be probed if sensitivities to the $\operatorname{BR}\left(t \rightarrow H^{ \pm} b\right)>0.01$ were achieved. However, a large part of the region roughly corresponding to $|Y|<0.32$ and $|X|<20$ (which is also not excluded by $b \rightarrow s \gamma$ ) would require a sensitivity to $\operatorname{BR}\left(t \rightarrow H^{ \pm} b\right)<0.01$ in order to be probed with the current search strategy for $t \rightarrow H^{ \pm} b$ and this is probably unlikely in the $8 \mathrm{TeV}$ run of the LHC.

Increased sensitivity to the plane of $[X, Y]$ can be achieved by requiring a $b$-tag on the jets which originate from the decay of $H^{ \pm}$. In Figs. 17 and 18, for $m_{H^{ \pm}}=80 \mathrm{GeV}$ and $m_{H^{ \pm}}=120 \mathrm{GeV}$, respectively, we show contours of

$$
\mathrm{BR}\left(t \rightarrow H^{ \pm} b\right) \times \mathrm{BR}\left(H^{ \pm} \rightarrow c b\right) .
$$

With the extra $b$-tag, as advocated previously (see eq. (12) in [41]), the sensitivity should reach $\mathrm{BR}\left(t \rightarrow H^{ \pm} b\right) \times \mathrm{BR}\left(H^{ \pm} \rightarrow c b\right)>0.5 \%$, and perhaps as low as $0.2 \%$. In the latter case, one can see from Figs. 17 (left) and 18 (right) that a large part of the regions of $|X|<6$ (for $m_{H^{ \pm}}=120 \mathrm{GeV}$ ) and $|X|<4$ (for $m_{H^{ \pm}}=80 \mathrm{GeV}$ ) could be probed, even for $|Y|<0.2$. Therefore, there would be sensitivity to a sizeable region of the parameter space of $[X, Y]$ which is not excluded by $b \rightarrow s \gamma$, a result which is in contrast to the above case where no $b$-tag is applied to the $b$-jets originating from $H^{ \pm}$. We encourage here a dedicated search for $t \rightarrow H^{ \pm} b$ and $H^{ \pm} \rightarrow c b$ by the Tevatron and LHC collaborations. Such a search would be a well-motivated extension and application of the searches which have already been carried out in [35] and [36] and would offer the possibility of increased sensitivity to the fermionic couplings and mass of $H^{ \pm}$in not only the MDHM/A2HDM but also the 2HDM-III.

\subsection{Direct $H^{ \pm}$production}

Another possibility to produce $H^{ \pm}$states in our scenario is via $c \bar{b}$ (and $b \bar{c}$ ) fusion in hadronhadron collisions. Since neither of the antiquarks in the initial state is a valence state, there 

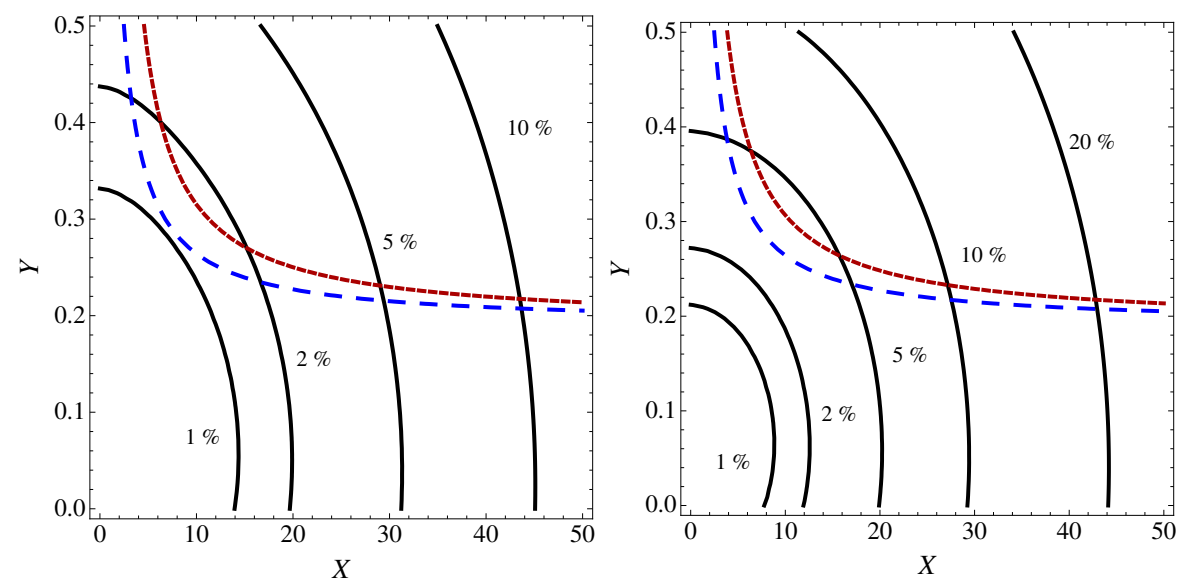

Figure 16. Contours of the sum of $\mathrm{BR}\left(t \rightarrow H^{ \pm} b\right) \times \mathrm{BR}\left(H^{ \pm} \rightarrow c s\right)$ and $\mathrm{BR}\left(t \rightarrow H^{ \pm} b\right) \times \mathrm{BR}\left(H^{ \pm} \rightarrow\right.$ $c b$ ) in the plane $[X, Y]$ with $|Z|=0.1$, where $m_{H^{ \pm}}=120 \mathrm{GeV}$ (left panel) and $m_{H^{ \pm}}=80 \mathrm{GeV}$ (right panel).
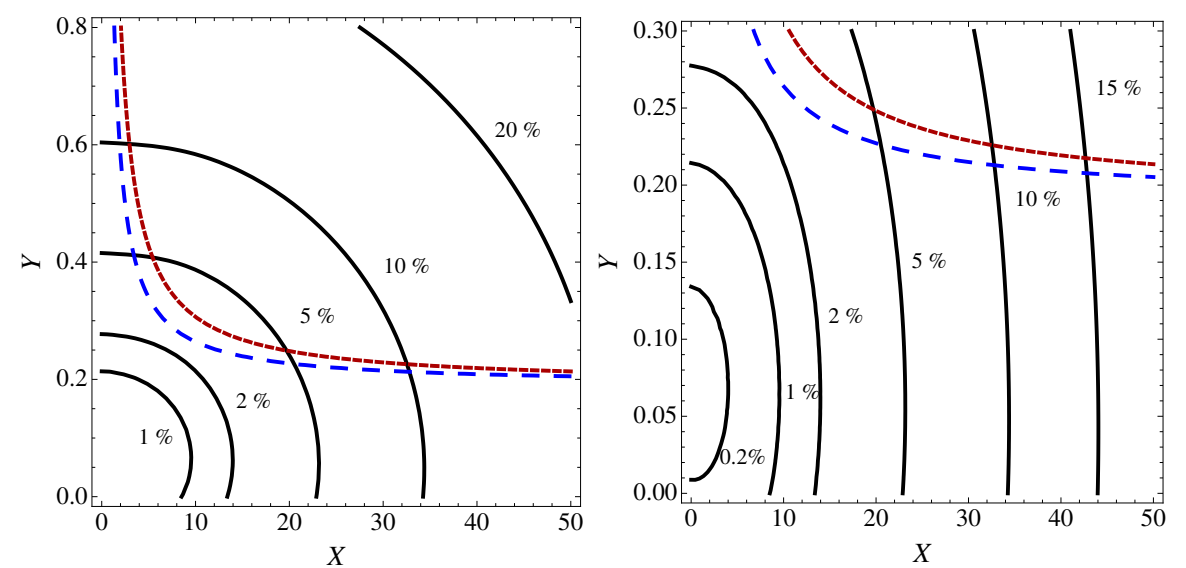

Figure 17. Contours of $\operatorname{BR}\left(t \rightarrow H^{ \pm} b\right) \times \mathrm{BR}\left(H^{ \pm} \rightarrow c b\right)$ in the plane $[X, Y]$ with $|Z|=0.1$ for $m_{H^{ \pm}}=80 \mathrm{GeV}$. The constraint $b \rightarrow s \gamma$ is shown as $|f B 1(X, Y)|<1.1$ for $\operatorname{Re}[f B 1(X, Y)]<0$ (red-dashed), and $|f B 1(X, Y)|<0.7$ is when $\operatorname{Re}[f B 1(X, Y)]<0$ (blue-dashed). We take $m_{s}(Q=$ $\left.m_{H^{ \pm}}\right)=0.055 \mathrm{GeV}$ and show the range $0<|Y|<0.8$ (left panel) and $0<|Y|<0.3$ (right panel).

is no intrinsic advantage in exploiting proton-antiproton coliisions at the Tevatron, further considering the much reduced energy and luminosity available at the FNAL accelerator with respect to the LHC. Hence, we focus our attention onto the latter. In fact, we can anticipate that, amongst all the energy and luminosity stages occurred or foreseen at the CERN machine, only the combinations $\sqrt{s}=8,14 \mathrm{TeV}$ and standard instantaneous luminosity of order $10^{33} \mathrm{~cm}^{-2} \mathrm{~s}^{-1}$ or higher are of relevance here. Furthermore, in the QCD polluted environment of the LHC, it is clear that one ought to attempt extracting a leptonic decay of our light charged Higgs boson. In the light of the results presented in the previous (sub)sections, the best option is afforded by $H^{ \pm}$decays into $\tau \nu$ pairs, eventually yielding an electron/muon (generically denoted by $l$ ) and missing (transverse) energy. In this case, the background is essentially due to the charged Drell-Yan (DY) channel giving 

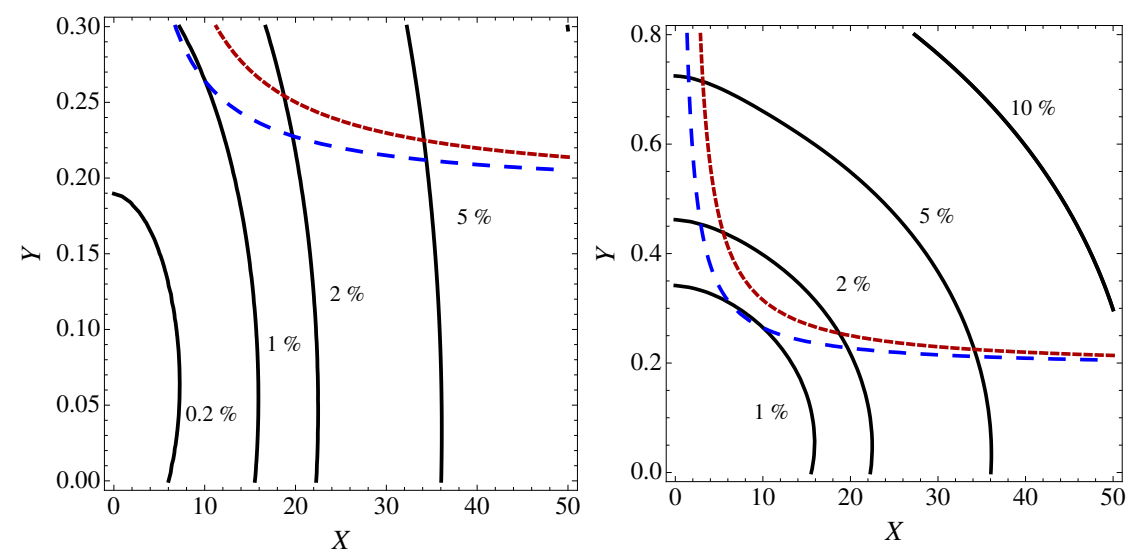

Figure 18. Contours of $\mathrm{BR}\left(t \rightarrow H^{ \pm} b\right) \times \mathrm{BR}\left(H^{ \pm} \rightarrow c b\right)$ in the plane $[X, Y]$ with $|Z|=0.1$ for $m_{H^{ \pm}}=120 \mathrm{GeV}$. The constraint $b \rightarrow s \gamma$ is shown as $|f B 1(X, Y)|<1.1$ for $\operatorname{Re}[f B 1(X, Y)]<0$ (red-dashed), and $|f B 1(X, Y)|<0.7$ is when $\operatorname{Re}[f B 1(X, Y)]<0$ (blue-dashed). We take $m_{s}(Q=$ $\left.m_{H^{ \pm}}\right)=0.055 \mathrm{GeV}$ and show the range $0<|Y|<0.8$ (left panel) and $0<|Y|<0.3$ (right panel).

$\tau \nu$ pairs (i.e., via $W^{ \pm}$production and decay), which is in fact irreducible.

Figs. 19-20 display the differential distribution for the signal $S$ (i.e. $c \bar{b} \rightarrow H^{+} \rightarrow$ $\tau^{+} \nu+$ c.c.) and the background $B$ (i.e., $c \bar{b} \rightarrow W^{+} \tau^{+} \nu+$ c.c), the former for three mass choices $\left(m_{H^{ \pm}}=80,120 \text { and } 160 \mathrm{GeV}\right)^{10}$. As the invariant mass of the final state is not reconstructible, given the missing longitudinal momemtum, we plot the transverse mass $M_{T} \equiv \sqrt{\left(E_{l}^{T}+E_{\text {miss }}^{T}\right)^{2}-\left(p_{l}^{x}+p_{\text {miss }}^{x}\right)^{2}-\left(p_{l}^{y}+p_{\text {miss }}^{y}\right)^{2}}$, where $E^{T}$ represents missing energy/momentum (as we consider the electron and muon massless) in the transverse plane and $p_{x, y}$ are the two components therein (assuming that the proton beams are directed along the $z$ axis). Clearly, the backgound dominance over the signal is evident whenever $m_{H^{ \pm}} \approx M_{W^{ \pm}}$. However, the larger the charged Higgs boson mass with respect to the gauge boson one, the more important the signal becomes relatively to the background.

In order to establish whether it is possible to extract the direct $H^{ \pm}$signal, or indeed constrain it, we show in Figs. 21-Figs. 22 the significance of the signal, defined as $S / \sqrt{B}$, as a function of the collider integrated luminosity $L$, where $S=L \sigma\left(c \bar{b} \rightarrow H^{+} \rightarrow \tau^{+} \nu+\right.$ c.c. $)$ and $B=L \sigma\left(c \bar{b} \rightarrow W^{+} \tau^{+} \nu+c . c.\right)$. Here, we have restricted both the signal and background yield to the mass regions $\left|m_{H^{ \pm}}-M_{T}\right|<10 \mathrm{GeV}$, where, again, we have taken $m_{H^{ \pm}}=80,120$ and $160 \mathrm{GeV}$. The $\tau$ decay rates into electron/muons are included and we assumed $90 \%$ efficiency in $e / \mu$-identification. It is clear that exclusion (significance equal to 2), evidence (significance equal to 3 ) and discovery (significance equal to 5 ) can all be attained at both the 8 and $14 \mathrm{TeV}$ energy stages, for accessible luminosity samples, so long that the $H^{ \pm}$mass is significantly larger than the $W^{ \pm}$one. In both machine configurations, corresponding event rates are always substantial.

\footnotetext{
${ }^{10}$ Notice that interference effects are negligible between the two, owing to the very narrow width of the charged Higgs boson.
} 

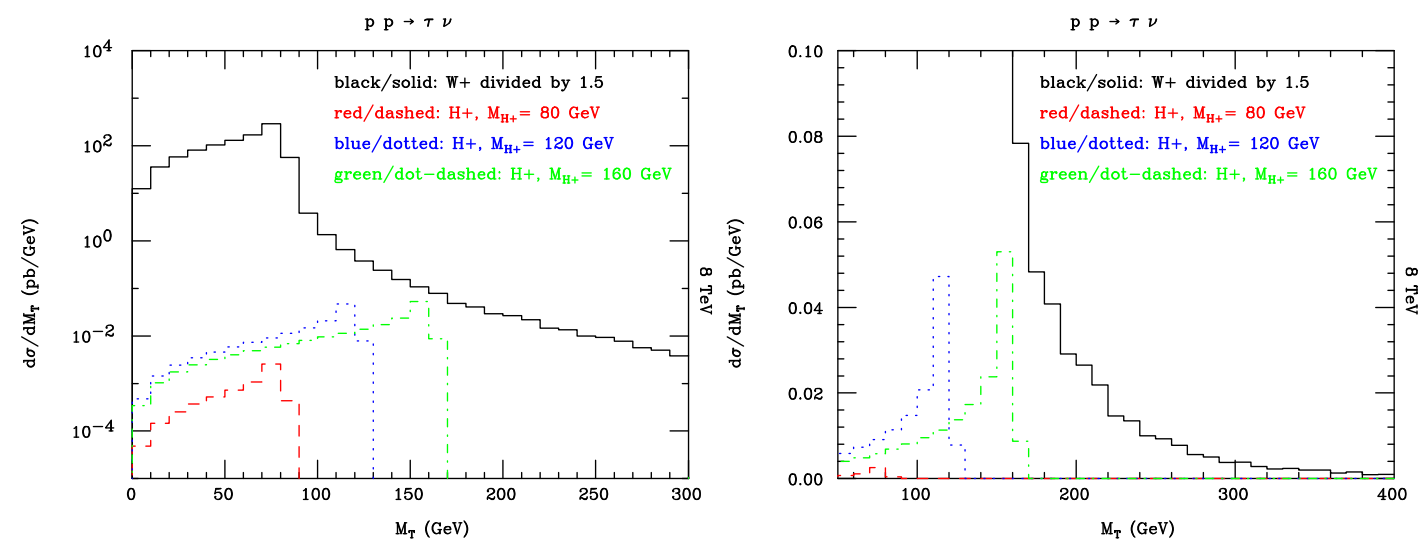

Figure 19. Differential distributions in transverse mass $M_{T}$ for signal and background (the former for three $H^{ \pm}$mass values) in logaritmic (top) and linear (bottom) scale. Here, $\sqrt{s}=8 \mathrm{TeV}$.
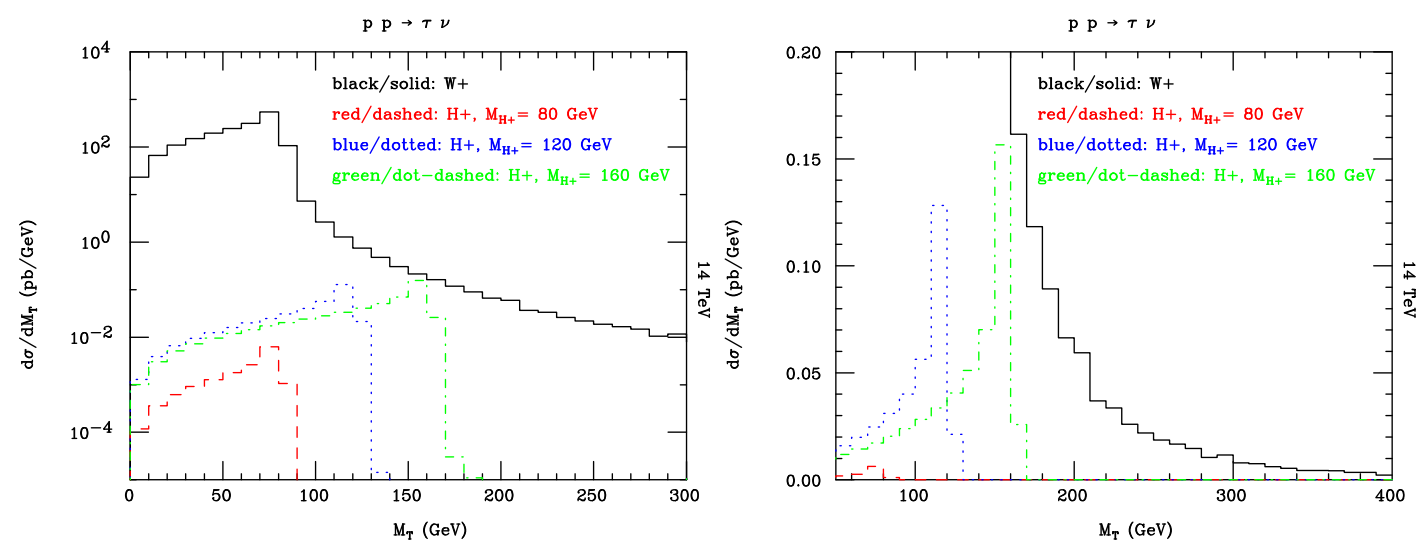

Figure 20. The same as Fig. 19, but for $\sqrt{s}=14 \mathrm{TeV}$.
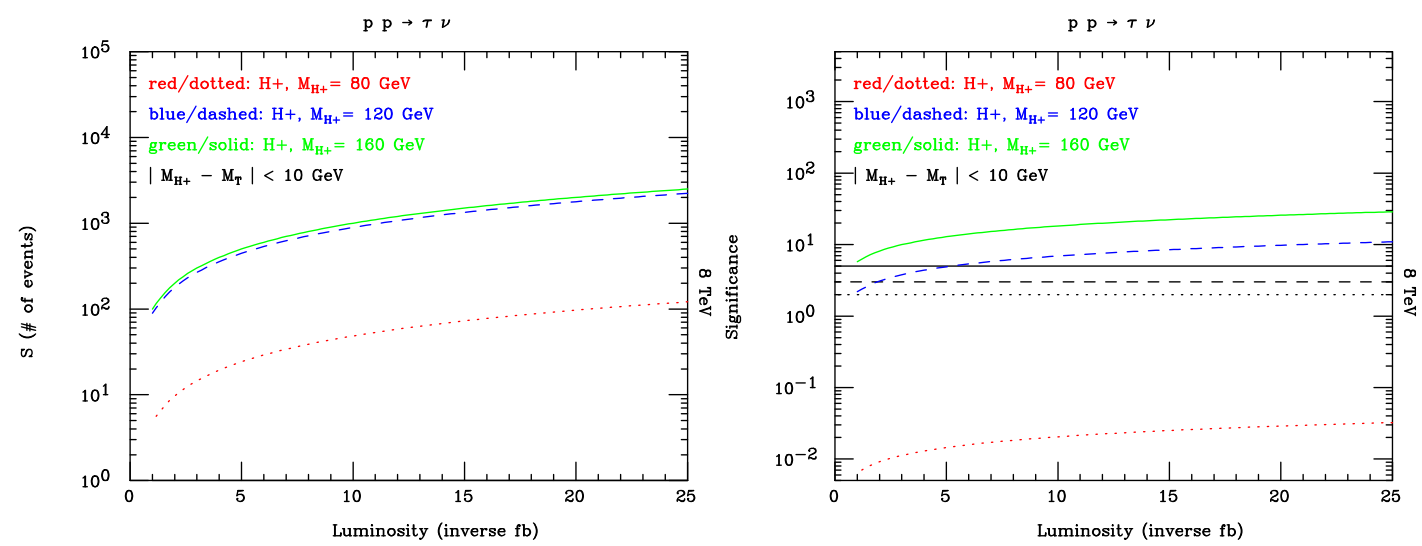

Figure 21. Number of events (top) and significance (bottom) of the signal (for three $H^{ \pm}$mass values) as a function of the luminosity. Here, $\sqrt{s}=8 \mathrm{TeV}$. The horizontal solid(dashed)[dotted] line corresponds to a significance of 5(3)[2]. 

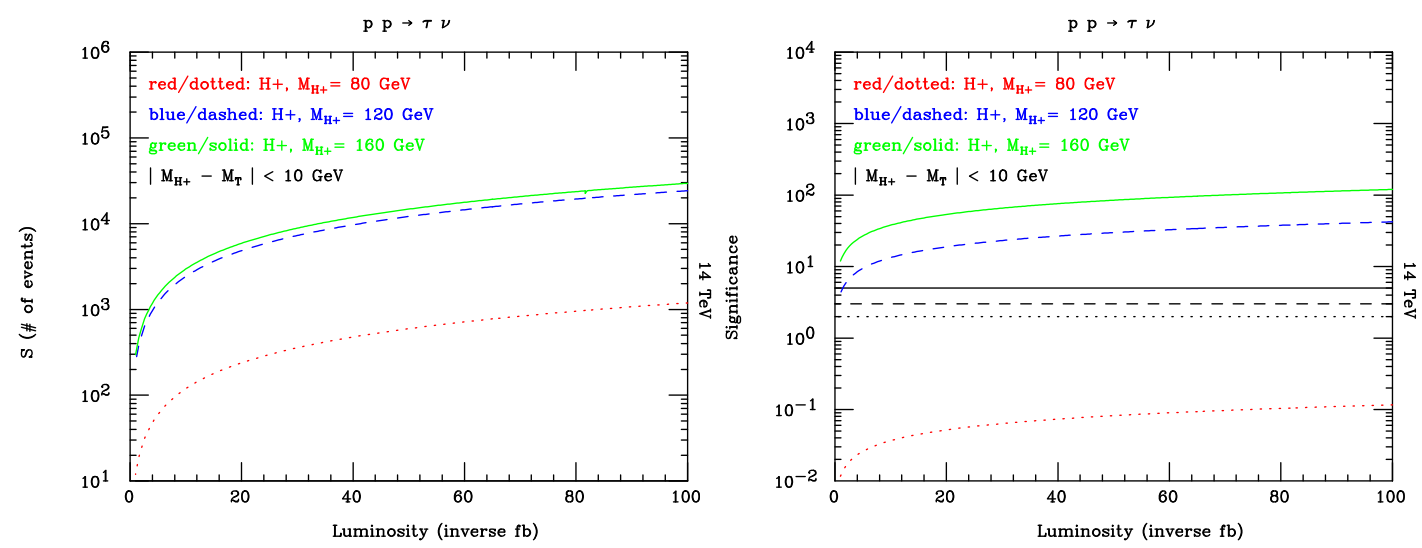

Figure 22. The same as Fig. 21, but for $\sqrt{s}=14 \mathrm{TeV}$.

\section{Conclusions}

Light charged Higgs bosons $\left(H^{ \pm}\right)$are being searched for in the decays of top quarks $(t \rightarrow$ $\left.H^{ \pm} b\right)$ at the Tevatron and LHC. Separate searches are being carried out for the decay channels $H^{ \pm} \rightarrow c s$ and $H^{ \pm} \rightarrow \tau \nu$, with comparable sensitivity to the mass and fermionic couplings of $H^{ \pm}$. The searches for $H^{ \pm} \rightarrow c s$ in [35] and [36] look for a peak at $m_{H^{ \pm}}$in the dijet invariant mass distribution, with the assumption that neither of the quarks is a $b$ quark.

In some models with two or more Higgs doublets (the 2HDM and a MHDM with three or more scalar doublets) the BR for $H^{ \pm} \rightarrow c b$ can be as large as $80 \%$. Here, in our model (2HDM-III), $\mathrm{BR}\left(H^{ \pm} \rightarrow c b\right)$ could be as large as $90 \%$. Moreover, such a $H^{ \pm}$could be light enough to be produced via $t \rightarrow H^{ \pm} b$ as well as respect the stringent constraints from $b \rightarrow s \gamma$ on both $m_{H^{ \pm}}$and the fermionic couplings of $H^{ \pm}$. This is in contrast to the $H^{ \pm}$ state in other 2HDMs for which a large BR for $H^{ \pm} \rightarrow c b$ is possible but for which one expects $m_{H^{ \pm}}>m_{t}$ in order to comply with the measured value of $b \rightarrow s \gamma$. Along the lines indicated by previous literature, in the context of the 2HDM-III with a four-zero Yukawa texture, we suggested that a dedicated search for $t \rightarrow H^{ \pm} b$ and $H^{ \pm} \rightarrow c b$ would probe values of the fermionic couplings of $H^{ \pm}$that are currently not testable. Such a search would require a $b$-tag of one of the jets originating from $H^{ \pm}$and would further allow one to reach a higher sensitivity to a smaller value of the $\operatorname{BR}\left(t \rightarrow H^{ \pm} b\right)$ than that obtained in the ongoing searches, which currently do not make use of this additional $b$-tag.

Finally, we have shown that a $H^{ \pm}$state of the 2HDM-III can also be produced directy from $c b$ fusion, followed by a decay into $\tau \nu$ pairs, assuming semi-leptonic decays (to contrast the otherwise overwhelming QCD background), albeit only at the LHC, which should have sensitivity to it already at $8 \mathrm{TeV}$ and, indeed, full coverage at $14 \mathrm{TeV}$, so long that $m_{H^{ \pm}}$ is $120 \mathrm{GeV}$ or above.

We have reached these conclusions over the 2HDM-III parameter space that we have shown to survive a long list of constraints emerging from $B$-physics, namely: $\mu-e$ universality in $\tau$ decays, several leptonic $B$-decays $\left(B \rightarrow \tau \nu, D \rightarrow \mu \nu\right.$ and $\left.D_{s} \rightarrow l \nu\right)$, the semi-leptonic transition $B \rightarrow D \tau \nu$, plus $B \rightarrow X_{s} \gamma$, including $B^{0}-\bar{B}^{0}$ mixing, $B_{s} \rightarrow \mu^{+} \mu^{-}$ 
and the radiative decay $Z \rightarrow b \bar{b}$.

The outlook is therefore clear. Depending on the search channel, both the Tevatron and the LHC have the potential to constrain or else discover the 2HDHM-III supplemented by a four-zero Yukawa texture including non-vanishing off-diagonal terms in the Yukawa matrices. We are now calling on our experimental colleagues to achieve this, as the search strategies recommended here are easily implementable and pursuable.

\section{Acknowledgements}

S.M. is supported in part through the NExT Institute. J. H.-S. thanks the University of Southampton and the Rutherford Appleton Laboratory for hospitality during his visit to the NExT Institute where part of this work was carried out. J. H.-S, A. R.-S and R. N.-P acknowledge the financial support of SNI, CONACYT, SEP-PROMEP and VIEP-BUAP. We all thank A.G. Akeroyd for innumerable constructive and useful discussions.

\section{References}

[1] S. L. Glashow, Partial Symmetries of Weak Interactions, Nucl. Phys. 22, (1961) 579; S. Weinberg, A Model of Leptons, Phys. Rev. Lett. 19, (1967) 1264; A. Salam, Weak and Electromagnetic Interactions, Conf. Proc. C 680519, (1968) 367.

[2] V. D. Barger, J. L. Hewett and R. J. N. Phillips, New Constraints on the Charged Higgs Sector in Two Higgs Doublets Models, Phys. Rev. D 41, (1990) 3421. For a recent review see, G. C. Branco, P. M. Ferreira, L. Lavoura, M. N. Rebelo, M. Sher and J. P. Silva, Theory and phenomenology of two-Higgs-doublet models, Phys. Rept. 516, (2012) 1 [arXiv:1106.0034 [hep-ph]].

[3] J. F. Gunion, H. E. Haber, G. L. Kane and S. Dawson, The Higgs Hunter's Guide, Front. Phys. 80, (2000).

[4] For all kind of 2HDMs see, T. D. Lee, A Theory of Spontaneous T Violation, Phys. Rev. D 8, (1973) 1226; M. Aoki, S. Kanemura, K. Tsumura and K. Yagyu, Models of Yukawa interaction in the two Higgs doublet model, and their collider phenomenology, Phys. Rev. D 80, (2009) 015017. [arXiv:0902.4665 [hep-ph]].

[5] For 2HDM-I see, H. E. Haber, G. L. Kane and T. Sterling, The Fermion Mass Scale And Possible Effects Of Higgs Bosons On Experimental Observables, Nucl. Phys. B 161, (1979) 493; L. J. Hall and M. B. Wise, Flavor Changing Higgs - Boson Couplings, Nucl. Phys. B 187, (1981) 397.

[6] For 2HDM-II see, J. F. Donoghue and L. F. Li, Properties Of Charged Higgs Bosons, Phys. Rev. D 19, (1979) 945.

[7] For 2HDM-X and 2HDM-Y see, R. M. Barnett, G. Senjanovic, L. Wolfenstein and D. Wyler, Implications Of A Light Higgs Scalar, Phys. Lett. B 136, (1984) 191; R. M. Barnett, G. Senjanovic and D. Wyler, Tracking Down Higgs Scalars With Enhanced Couplings, Phys. Rev. D 30, (1984) 1529.

[8] Y. Grossman, Phenomenology of models with more than two Higgs doublets, Nucl. Phys. B 426, (1994) 355 [hep-ph/9401311]. 
[9] A. G. Akeroyd, Nonminimal neutral Higgs bosons at LEP-2, Phys. Lett. B 377, (1996) 95 [hep-ph/9603445]. A. G. Akeroyd, Fermiophobic and other nonminimal neutral Higgs bosons at the LHC, J. Phys. G 24, (1998) 1983 [hep-ph/9803324].

[10] A. G. Akeroyd and W. J. Stirling, Light charged Higgs scalars at high-energy e+ ecolliders," Nucl. Phys. B 447, (1995) 3.

[11] E. Ma, Utility of a Special Second Scalar Doublet, Mod. Phys. Lett. A 23, (2008) 647 [arXiv:0802.2917 [hep-ph]].

[12] E. Ma, Verifiable radiative seesaw mechanism of neutrino mass and dark matter, Phys. Rev. D 73, (2006) 077301 [hep-ph/0601225].

[13] R. Barbieri, L. J. Hall and V. S. Rychkov, Improved naturalness with a heavy Higgs: An Alternative road to LHC physics, Phys. Rev. D 74, (2006) 015007 [hep-ph/0603188].

[14] L. Lopez Honorez, E. Nezri, J. F. Oliver and M. H. G. Tytgat, The Inert Doublet Model: An Archetype for Dark Matter, JCAP 0702, (2007) 028 [hep-ph/0612275].

[15] S. L. Glashow and S. Weinberg, Natural Conservation Laws for Neutral Currents, Phys. Rev. D 15, (1977) 1958.

[16] A. Pich and P. Tuzon, Yukawa Alignment in the Two-Higgs-Doublet Model, Phys. Rev. D 80, (2009) 091702 [arXiv:0908.1554 [hep-ph]].

[17] Y. -F. Zhou, Texture of Yukawa coupling matrices in general two Higgs doublet model, J. Phys. G 30, (2004) 783 [hep-ph/0307240].

[18] S. Kanemura, T. Ota and K. Tsumura, Lepton flavor violation in Higgs boson decays under the rare tau decay results, Phys. Rev. D 73, (2006) 016006 [hep-ph/0505191].

[19] S. Kanemura, K. Matsuda, T. Ota, T. Shindou, E. Takasugi and K. Tsumura, Search for lepton flavor violation in the Higgs boson decay at a linear collider,' Phys. Lett. B 599, (2004) 83 [hep-ph/0406316].

[20] T. P. Cheng and M. Sher, Mass Matrix Ansatz and Flavor Nonconservation in Models with Multiple Higgs Doublets, Phys. Rev. D 35, (1987) 3484.

[21] D. Atwood, L. Reina and A. Soni, Phenomenology of two Higgs doublet models with flavor changing neutral currents, Phys. Rev. D 55, (1997) 3156 [hep-ph/9609279].

[22] J. L. Diaz-Cruz, R. Noriega-Papaqui and A. Rosado, Measuring the fermionic couplings of the Higgs boson at future colliders as a probe of a non-minimal flavor structure, Phys. Rev. D 71, (2005) 015014 [hep-ph/0410391].

[23] J. L. Diaz-Cruz, J. Hernandez-Sanchez, S. Moretti, R. Noriega-Papaqui and A. Rosado, Yukawa Textures and Charged Higgs Boson Phenomenology in the 2HDM-III, Phys. Rev. D 79, (2009) 095025 [arXiv:0902.4490 [hep-ph]].

[24] F. Mahmoudi and O. Stal, Flavor constraints on the two-Higgs-doublet model with general Yukawa couplings, Phys. Rev. D 81, (2010) 035016 [arXiv:0907.1791 [hep-ph]].

[25] H. Fritzsch, Calculating the Cabibbo Angle, Phys. Lett. B 70, (1977) 436.

[26] H. Fritzsch and Z. -z. Xing, Four zero texture of Hermitian quark mass matrices and current experimental tests, Phys. Lett. B 555, (2003) 63 [hep-ph/0212195].

[27] J. Hernandez-Sanchez, L. Lopez-Lozano, R. Noriega-Papaqui and A. Rosado, Couplings of quarks in the Partially Aligned 2HDM with a four-zero texture Yukawa matrix, Phys. Rev. $D$ 85, (2012) 071301 [arXiv:1106.5035 [hep-ph]]. 
[28] J. E. Barradas Guevara, F. C. Cazarez Bush, A. Cordero Cid, O. F. Felix Beltran, J. Hernandez Sanchez and R. Noriega Papaqui, Implications of Yukawa Textures in the decay $H^{+} \rightarrow W^{+} \gamma$ within the 2HDM-III," J. Phys. G 37, (2010) 115008 [arXiv:1002.2626 [hep-ph]]; A. Cordero-Cid, O. Felix-Beltran, J. Hernandez-Sanchez and R. Noriega-Papaqui, Implications of Yukawa texture in the charged Higgs boson phenomenology within 2HDM-III, PoS CHARGED 2010, (2010) 042 [arXiv:1105.4951 [hep-ph]].

[29] M. Gomez-Bock and R. Noriega-Papaqui, Flavor violating decays of the Higgs bosons in the THDM-III, J. Phys. G 32, (2006) 761 [hep-ph/0509353].

[30] J. Hernandez-Sanchez, C. G. Honorato, M. A. Perez and J. J. Toscano, The $\gamma \gamma \rightarrow \phi_{i} \phi_{j}$ processes in the type-III two-Higgs-doublet model, Phys. Rev. D 85, (2012) 015020 [arXiv:1108.4074 [hep-ph]].

[31] M. Aoki, R. Guedes, S. Kanemura, S. Moretti, R. Santos and K. Yagyu, Light Charged Higgs bosons at the LHC in 2HDMs, Phys. Rev. D 84, (2011) 055028 [arXiv:1104.3178 [hep-ph]].

[32] S. Moretti, Pair production of charged Higgs scalars from electroweak gauge boson fusion, J. Phys. G 28, (2002) 2567 [hep-ph/0102116]; S. Moretti, Improving the discovery potential of charged Higgs bosons at the Tevatron and Large Hadron Collider, Pramana 60, (2003) 369 [hep-ph/0205104].

[33] J. F. Gunion, H. E. Haber, F. E. Paige, W. -K. Tung and S. S. D. Willenbrock, Neutral and Charged Higgs Detection: Heavy Quark Fusion, Top Quark Mass Dependence and Rare Decays, Nucl. Phys. B 294, (1987) 621; J. L. Diaz-Cruz and O. A. Sampayo, Contribution of gluon fusion to the production of charged Higgs at hadron colliders, Phys. Rev. D 50, (1994) 6820; S. Moretti and D. P. Roy, Detecting heavy charged Higgs bosons at the LHC with triple b tagging, Phys. Lett. B 470, (1999) 209 [hep-ph/9909435]; D. J. Miller, S. Moretti, D. P. Roy and W. J. Stirling, Detecting heavy charged Higgs bosons at the CERN LHC with four b quark tags, Phys. Rev. D 61, (2000) 055011 [hep-ph/9906230].

[34] V. M. Abazov et al. [D0 Collaboration], Search for charged Higgs bosons in top quark decays, Phys. Lett. B 682, 278 (2009) [arXiv:0908.1811 [hep-ex]].

[35] T. Aaltonen et al. [CDF Collaboration], Search for charged Higgs bosons in decays of top quarks in $p$ anti-p collisions at $\sqrt{s}=1.96$ TeV, Phys. Rev. Lett. 103, 101803 (2009) [arXiv:0907.1269 [hep-ex]].

[36] G. Aad et al. [ATLAS Collaboration], A Search for a light charged Higgs boson decaying to $\mathrm{cs}^{-}$in pp collisions at $\sqrt{s}=7 \mathrm{TeV}$ with the ATLAS detector, ATLAS-CONF-2011-094 (July 2011).

[37] G. Aad et al. [ATLAS Collaboration], Search for charged Higgs bosons decaying via $\mathrm{H}^{+} \rightarrow \tau \nu$ in $t \bar{t}$ events using $4.6 \mathrm{fb}^{-1}$ of pp collision data at $\sqrt{(\mathrm{s})}=7 \mathrm{TeV}$ with the ATLAS detector, ATLAS-CONF-2012-011 (March 2012).

[38] S. Chatrchyan et al. [CMS Collaboration], Search for the charged Higgs boson with $H^{+} \rightarrow \tau^{+} \nu$ mode in top quark decays, CMS-PAS-HIG-11-008 (July 2011).

[39] A. G. Akeroyd, Hidden top quark decays to charged Higgs scalars at the Tevatron, hep-ph/9509203.

[40] A. G. Akeroyd, Three body decays of Higgs bosons at LEP-2 and application to a hidden fermiophobic Higgs, Nucl. Phys. B 544, (1999) 557 [hep-ph/9806337]. 
[41] A. G. Akeroyd, S. Moretti and J. Hernandez-Sanchez, Light charged Higgs bosons decaying to charm and bottom quarks in models with two or more Higgs doublets, Phys. Rev. D 85, (2012) 115002 [arXiv:1203.5769 [hep-ph]].

[42] H. E. Logan and D. MacLennan, Charged Higgs phenomenology in the flipped two Higgs doublet model, Phys. Rev. D 81, (2010) 075016 [arXiv:1002.4916 [hep-ph]].

[43] F. Borzumati and C. Greub, 2HDMs predictions for $\bar{B} \rightarrow X_{s} \gamma$ in NLO QCD, Phys. Rev. D 58, (1998) 074004 [hep-ph/9802391]; F. Borzumati and C. Greub, Two Higgs doublet model predictions for $\bar{B} \rightarrow X_{s} \gamma$ in NLO QCD: Addendum, Phys. Rev. D 59, (1999) 057501 [hep-ph/9809438].

[44] M. Misiak, H. M. Asatrian, K. Bieri, M. Czakon, A. Czarnecki, T. Ewerth, A. Ferroglia and P. Gambino et al., Estimate of $B\left(\bar{B} \rightarrow X_{s} \gamma\right)$ at $\mathcal{O}\left(\alpha_{s}^{2}\right)$, Phys. Rev. Lett. 98, (2007) 022002 [hep-ph/0609232].

[45] J. F. Gunion and H. E. Haber, The CP conserving two Higgs doublet model: The Approach to the decoupling limit, Phys. Rev. D 67, (2003) 075019 [hep-ph/0207010].

[46] T. Aaltonen et al. [CDF and D0 Collaborations], Evidence for a particle produced in association with weak bosons and decaying to a bottom-antibottom quark pair in Higgs boson searches at the Tevatron, Phys. Rev. Lett. 109, (2012) 071804 [arXiv:1207.6436 [hep-ex]]; S. Chatrchyan et al. [CMS Collaboration], Observation of a new boson at a mass of 125 GeV with the CMS experiment at the LHC, Phys. Lett. B 716, (2012) 30 [arXiv:1207.7235 [hep-ex]]; G. Aad et al. [ATLAS Collaboration], Observation of a new particle in the search for the Standard Model Higgs boson with the ATLAS detector at the LHC, Phys. Lett. B 716, (2012) 1 [arXiv:1207.7214 [hep-ex]].

[47] S. Kanemura and H. -A. Tohyama, Nondecoupling effects of Higgs bosons on $e^{+} e^{-} \rightarrow W(L)^{+} W(L)^{-}$in the two doublet model, Phys. Rev. D 57, (1998) 2949 [hep-ph/9707454]; S. Kanemura, Enhancement of loop induced $H^{ \pm} W^{\mp} Z^{0}$ vertex in two Higgs doublet model, Phys. Rev. D 61, (2000) 095001 [hep-ph/9710237]; S. Kanemura, Possible enhancement of the $e^{+} e^{-} \rightarrow H^{ \pm} W^{\mp}$ cross-section in the two Higgs doublet model, Eur. Phys. J. C 17, (2000) 473 [hep-ph/9911541].

[48] J. L. Diaz-Cruz, R. Noriega-Papaqui and A. Rosado, Mass matrix ansatz and lepton flavor violation in the THDM-III, Phys. Rev. D 69, (2004) 095002 [hep-ph/0401194].

[49] O. Félix-Beltrán, F. F. González-Canales, J. Hernández-Sánchez, S. Moretti, R. Noriega-Papaqui and A. Rosado, work in progress.

[50] J. Bijnens, J. Lu and J. Rathsman, Constraining General Two Higgs Doublet Models by the Evolution of Yukawa Couplings, JHEP 1205, (2012) 118 [arXiv:1111.5760 [hep-ph]].

[51] R. G. Roberts, A. Romanino, G. G. Ross and L. Velasco-Sevilla, Precision test of a fermion mass texture, Nucl. Phys. B 615, (2001) 358 [hep-ph/0104088].

[52] M. Frigerio, S. Kaneko, E. Ma and M. Tanimoto, Quaternion family symmetry of quarks and leptons, Phys. Rev. D 71, (2005) 011901 [hep-ph/0409187].

[53] P. H. Frampton and S. Matsuzaki, Renormalizable A(4) Model for Lepton Sector, arXiv:0806.4592 [hep-ph]; P. H. Frampton, T. W. Kephart and S. Matsuzaki, Simplified Renormalizable T-prime Model for Tribimaximal Mixing and Cabibbo Angle, Phys. Rev. D 78, (2008) 073004 [arXiv:0807.4713 [hep-ph]].

[54] T. Fukuyama, H. Sugiyama and K. Tsumura, Phenomenology in the Higgs Triplet Model 
with the $A_{4}$ Symmetry, Phys. Rev. D 82, (2010) 036004 [arXiv:1005.5338 [hep-ph]]; T. Fukuyama, H. Sugiyama and K. Tsumura, Phenomenology in the Zee Model with the $A_{4}$ Symmetry, Phys. Rev. D 83, (2011) 056016 [arXiv:1012.4886 [hep-ph]].

[55] A. Aranda, C. Bonilla, R. Ramos and A. D. Rojas, Model of flavor with quaternion symmetry, Phys. Rev. D 84, (2011) 016009 [arXiv:1105.6373 [hep-ph]]; A. Aranda, C. Bonilla and A. D. Rojas, Neutrino masses generation in a $Z_{4}$ model, Phys. Rev. D 85, (2012) 036004 [arXiv:1110.1182 [hep-ph]].

[56] A. Aranda, C. Bonilla and J. L. Diaz-Cruz, Three generations of Higgses and the cyclic groups, Phys. Lett. B 717, (2012) 248 [arXiv:1204.5558 [hep-ph]].

[57] G. C. Branco, D. Emmanuel-Costa and C. Simoes, Nearest-Neighbour Interaction from an Abelian Symmetry and Deviations from Hermiticity, Phys. Lett. B 690, (2010) 62 [arXiv:1001.5065 [hep-ph]]. F. J. Botella, G. C. Branco and M. N. Rebelo, Invariants and Flavour in the General Two-Higgs Doublet Model, arXiv:1210.8163 [hep-ph].

[58] H. E. Logan and D. MacLennan, Charged Higgs phenomenology in the lepton-specific two Higgs doublet model, Phys. Rev. D 79, (2009) 115022 [arXiv:0903.2246 [hep-ph]];

M. Krawczyk and D. Temes, 2HDM(II) radiative corrections in leptonic tau decays, Eur. Phys. J. C 44, (2005) 435 [hep-ph/0410248].

[59] Y. -S. Tsai, Decay Correlations of Heavy Leptons in $e^{+} e^{-} \rightarrow \ell^{+} \ell^{-}$, Phys. Rev. D 4, (1971) 2821 [Erratum-ibid. D 13, (1976) 771].

[60] B. Aubert et al. [BABAR Collaboration], Measurements of Charged Current Lepton Universality and $\left|V_{u s}\right|$ using Tau Lepton Decays to $e^{-} \bar{\nu}_{e} \nu_{\tau}, \mu^{-} \bar{\nu}_{\mu} \nu_{\tau}, \pi^{-} \nu_{\tau}$ and $K^{-} \nu_{\tau}$, Phys. Rev. Lett. 105, (2010) 051602 [arXiv:0912.0242 [hep-ex]].

[61] M. Jung, A. Pich and P. Tuzon, Charged-Higgs phenomenology in the Aligned two-Higgs-doublet model, JHEP 1011, (2010) 003 [arXiv:1006.0470 [hep-ph]].

[62] O. Deschamps, S. Descotes-Genon, S. Monteil, V. Niess, S. T'Jampens and V. Tisserand, The Two Higgs Doublet of Type II facing flavour physics data, Phys. Rev. D 82, (2010) 073012 [arXiv:0907.5135 [hep-ph]].

[63] B. I. Eisenstein et al. [CLEO Collaboration], Precision Measurement of $B\left(D^{+} \rightarrow \mu^{+} \nu\right)$ and the Pseudoscalar Decay Constant $f_{D^{+}}$, Phys. Rev. D 78, (2008) 052003 [arXiv:0806.2112 [hep-ex]].

[64] A. Matyja et al. [Belle Collaboration], Observation of $B^{0} \rightarrow D^{*-} \tau^{+} \nu_{\tau}$ decay at Belle, Phys. Rev. Lett. 99, (2007) 191807 [arXiv:0706.4429 [hep-ex]].

[65] B. Aubert et al. [BABAR Collaboration], Measurement of the Semileptonic Decays $\bar{B} \rightarrow D \tau^{-} \bar{\nu}_{\tau}$ and $\bar{B} \rightarrow D^{*} \tau^{-} \bar{\nu}_{\tau}$, Phys. Rev. D 79, (2009) 092002 [arXiv:0902.2660 [hep-ex]].

[66] J. P. Lees et al. [BaBar Collaboration], Evidence for an excess of $\bar{B} \rightarrow D^{(*)} \tau^{-} \bar{\nu}_{\tau}$ decays, Phys. Rev. Lett. 109, (2012) 101802 [arXiv:1205.5442 [hep-ex]].

[67] A. Crivellin, C. Greub and A. Kokulu, Explaining $B \rightarrow D \tau \nu, B \rightarrow D^{*} \tau \nu$ and $B \rightarrow \tau \nu$ in a 2HDM of type III," Phys. Rev. D 86, (2012) 054014 [arXiv:1206.2634 [hep-ph]].

[68] E. Barberio et al. [Heavy Flavor Averaging Group Collaboration], Averages of b-hadron and c-hadron Properties at the End of 2007, arXiv:0808.1297 [hep-ex].

[69] G. M. de Divitiis, R. Petronzio and N. Tantalo, Quenched lattice calculation of semileptonic heavy-light meson form factors, JHEP 0710, (2007) 062 [arXiv:0707.0587 [hep-lat]]. 
[70] M. Ciuchini, G. Degrassi, P. Gambino and G. F. Giudice, Next-to-leading QCD corrections to $\bar{B} \rightarrow X_{s} \gamma$ : Standard model and two Higgs doublet model, Nucl. Phys. B 527, (1998) 21 [hep-ph/9710335].

[71] P. Ciafaloni, A. Romanino and A. Strumia, Two loop QCD corrections to charged Higgs mediated $b \rightarrow$ s $\gamma$ decay, Nucl. Phys. B 524, (1998) 361 [hep-ph/9710312].

[72] G. Degrassi and P. Slavich, QCD Corrections in two-Higgs-doublet extensions of the Standard Model with Minimal Flavor Violation, Phys. Rev. D 81, (2010) 075001 [arXiv:1002.1071 [hep-ph]].

[73] T. Hermann, M. Misiak and M. Steinhauser, $\bar{B} \rightarrow X_{s} \gamma$ in the Two Higgs Doublet Model up to Next-to-Next-to-Leading Order in QCD, JHEP 1211, (2012) 036 [arXiv:1208.2788 [hep-ph]].

[74] S. Chen et al. [CLEO Collaboration], Branching fraction and photon energy spectrum for $b \rightarrow s \gamma$, Phys. Rev. Lett. 87, (2001) 251807 [hep-ex/0108032].

[75] K. Abe et al. [Belle Collaboration], A Measurement of the branching fraction for the inclusive $\bar{B} \rightarrow X_{s} \gamma$ decays with Belle," Phys. Lett. B 511, (2001) 151 [hep-ex/0103042].

[76] A. Limosani et al. [Belle Collaboration], Measurement of Inclusive Radiative B-meson Decays with a Photon Energy Threshold of 1.7-GeV, Phys. Rev. Lett. 103, (2009) 241801 [arXiv:0907.1384 [hep-ex]].

[77] J. P. Lees et al. [BaBar Collaboration], Precision Measurement of the $B \rightarrow X_{s} \gamma$ Photon Energy Spectrum, Branching Fraction, and Direct $C P$ Asymmetry $A_{C P}\left(B \rightarrow X_{s+d} \gamma\right)$, Phys. Rev. Lett. 109, (2012) 191801 [arXiv:1207.2690 [hep-ex]].

[78] J. P. Lees et al. [BaBar Collaboration], Exclusive Measurements of $b \rightarrow s \gamma$ Transition Rate and Photon Energy Spectrum, Phys. Rev. D 86, (2012) 052012 [arXiv:1207.2520 [hep-ex]].

[79] B. Aubert et al. [BABAR Collaboration], Measurement of the $\bar{B} \rightarrow X_{s} \gamma$ branching fraction and photon energy spectrum using the recoil method, Phys. Rev. D 77, (2008) 051103 [arXiv:0711.4889 [hep-ex]].

[80] Z. -j. Xiao and L. Guo, $\bar{B}^{0}-B^{0}$ mixing and $\bar{B} \rightarrow X_{s} \gamma$ decay in the third type 2HDM: Effects of NLO QCD contributions, Phys. Rev. D 69, (2004) 014002 [hep-ph/0309103].

[81] N. Cabibbo and L. Maiani, The Lifetime of Charmed Particles, Phys. Lett. B 79, (1978) 109; Y. Nir, The Mass Ratio $m_{c} / m_{b}$ in Semileptonic B Decays," Phys. Lett. B 221, (1989) 184.

[82] A. Ali and C. Greub, Inclusive photon energy spectrum in rare B decays, Z. Phys. C 49, (1991) 431; Photon energy spectrum in $\bar{B} \rightarrow X_{s} \gamma$ and comparison with data, Phys. Lett. $B$ 259, (1991) 182; 361, 146 (1995) [hep-ph/9506374]; N. Pott, Bremsstrahlung corrections to the decay $b \rightarrow s \gamma$, Phys. Rev. D 54, (1996) 938 [hep-ph/9512252].

[83] M. Trott and M. B. Wise, On theories of enhanced CP violation in $B_{s, d}$ meson mixing," JHEP 1011, (2010) 157 [arXiv:1009.2813 [hep-ph]].

[84] D. Bowser-Chao, K. -m. Cheung and W. -Y. Keung, Phase effect of a general two Higgs doublet model in $b \rightarrow s \gamma$, Phys. Rev. D 59, (1999) 115006 [hep-ph/9811235].

[85] J. Urban, F. Krauss, U. Jentschura and G. Soff, Next-to-leading order QCD corrections for the $B^{0}-\bar{B}^{0}$ mixing with an extended Higgs sector, Nucl. Phys. B 523, (1998) 40 [hep-ph/9710245]. 
[86] H. E. Haber and H. E. Logan, Radiative corrections to the Z Zb̄ vertex and constraints on extended Higgs sectors, Phys. Rev. D 62, (2000) 015011 [hep-ph/9909335]; H. E. Logan, Radiative corrections to the $Z b \bar{b}$ vertex and constraints on extended Higgs sectors, hep-ph/9906332.

[87] J. Beringer et al. [Particle Data Group Collaboration], Review of Particle Physics (RPP), Phys. Rev. D 86, (2012) 010001.

[88] RAaij et al. [LHCb Collaboration], First evidence for the decay Bs -i mu+ mu-, Phys. Rev. Lett. 110, 021801 (2013) [arXiv:1211.2674 [Unknown]].

[89] A. J. Buras, M. V. Carlucci, S. Gori and G. Isidori, Higgs-mediated FCNCs: Natural Flavour Conservation vs. Minimal Flavour Violation,' JHEP 1010, 009 (2010) [arXiv:1005.5310 [hep-ph]].

[90] H. E. Logan and U. Nierste, $B\left(s, d^{)} \rightarrow \ell^{+} \ell^{-}\right.$in a two Higgs doublet model, Nucl. Phys. $B$ 586, 39 (2000) [hep-ph/0004139].

[91] C. -S. Huang, W. Liao, Q. -S. Yan and S. -H. Zhu, $B_{s} \rightarrow$ lepton + lepton - in a general 2 HDM and MSSM, Phys. Rev. D 63, 114021 (2001) [Erratum-ibid. D 64, 059902 (2001)] [hep-ph/0006250].

[92] A. Crivellin, A. Kokulu and C. Greub, Flavor-phenomenology of two-Higgs-doublet models with generic Yukawa structure, arXiv:1303.5877 [hep-ph].

[93] W. Altmannshofer, A. J. Buras, S. Gori, P. Paradisi and D. M. Straub, Anatomy and Phenomenology of FCNC and CPV Effects in SUSY Theories, Nucl. Phys. B 830, 17 (2010) [arXiv:0909.1333 [hep-ph]].

[94] A. Dedes, A. Pilaftsis and, Resummed effective Lagrangian for Higgs mediated FCNC interactions in the CP violating MSSM, Phys. Rev. D 67, 015012 (2003) [hep-ph/0209306].

[95] A. Bazavov et al. [Fermilab Lattice and MILC Collaborations], B- and D-meson decay constants from three-flavor lattice QCD, Phys. Rev. D 85, 114506 (2012) [arXiv:1112.3051 [hep-lat]].

[96] J. Laiho, E. Lunghi and R. S. Van de Water, Lattice QCD inputs to the CKM unitarity triangle analysis, Phys. Rev. D 81, (2010) 034503 [arXiv:0910.2928 [hep-ph]].

[97] G. Colangelo, S. Durr, A. Juttner, L. Lellouch, H. Leutwyler, V. Lubicz, S. Necco and C. T. Sachrajda et al., Review of lattice results concerning low energy particle physics, Eur. Phys. J. C 71, (2011) 1695 [arXiv:1011.4408 [hep-lat]].

[98] R. M. Barnett, R. Cruz, J. F. Gunion and B. Hubbard, Charged Higgs bosons at the SSC, Phys. Rev. D 47, (1993) 1048.

[99] [LEP Higgs Working Group for Higgs boson searches and ALEPH and DELPHI and L3 and OPAL Collaborations], Search for charged Higgs bosons: Preliminary combined results using LEP data collected at energies up to 209-GeV, hep-ex/0107031.

[100] A. Ferrari [ATLAS Collaboration], Search strategies for charged Higgs bosons in ATLAS, PoS CHARGED2010, (2010) 010 ; ATL-PHYS-PUB-2010-009.

[101] S. Moretti and W. J. Stirling, Contributions of below threshold decays to MSSM Higgs branching ratios, Phys. Lett. B 347, (1995) 291 [Erratum-ibid. B 366, (1996)] 451 [hep-ph/9412209, hep-ph/9511351]; A. Djouadi, J. Kalinowski and P. M. Zerwas, Two and three-body decay modes of SUSY Higgs particles, Z. Phys. C 70, (1996) 435 [hep-ph/9511342]. 
[102] J. Abdallah et al. [DELPHI Collaboration], Search for charged Higgs bosons at LEP in general two Higgs doublet models, Eur. Phys. J. C 34, (2004) 399 [hep-ex/0404012]; G. Abbiendi et al. [OPAL Collaboration], Search for Charged Higgs Bosons in e+eCollisions at sqrts(s) = 189-209 GeV, Eur. Phys. J. C 72, (2012) 2076 [arXiv:0812.0267 [hep-ex]].

[103] D. Toussaint, Renormalization Effects From Superheavy Higgs Particles, Phys. Rev. D 18, (1978) 1626; S. Bertolini, Quantum Effects In A Two Higgs Doublet Model Of The Electroweak Interactions, Nucl. Phys. B 272, (1986) 77.

[104] M. E. Peskin and T. Takeuchi, A New constraint on a strongly interacting Higgs sector, Phys. Rev. Lett. 65, (1990) 964; M. E. Peskin and T. Takeuchi, Estimation of oblique electroweak corrections, Phys. Rev. D 46, (1992) 381.

[105] S. Kanemura, Y. Okada, H. Taniguchi and K. Tsumura, Indirect bounds on heavy scalar masses of the two-Higgs-doublet model in light of recent Higgs boson searches, Phys. Lett. B 704, (2011) 303. [arXiv:1108.3297 [hep-ph]].

[106] P. H. Chankowski, M. Krawczyk and J. Zochowski, Implications of the precision data for very light Higgs boson scenario in 2HDM(II), Eur. Phys. J. C 11, (1999) 661 [hep-ph/9905436]; A. Wahab El Kaffas, P. Osland and O. M. Ogreid, Constraining the Two-Higgs-Doublet-Model parameter space, Phys. Rev. D 76, (2007) 095001 [arXiv:0706.2997 [hep-ph]].

[107] W. Grimus, L. Lavoura, O. M. Ogreid and P. Osland, The Oblique parameters in multi-Higgs-doublet models, Nucl. Phys. B 801, (2008) 81 [arXiv:0802.4353 [hep-ph]].

[108] CDF Collaboration, CDF Note 10104 (June 2010).

[109] R. Dermisek and J. F. Gunion, Many Light Higgs Bosons in the NMSSM, Phys. Rev. D 79, (2009) 055014. [arXiv:0811.3537 [hep-ph]].

[110] G. Burdman, C. E. F. Haluch and R. D. Matheus, Is the LHC Observing the Pseudo-scalar State of a Two-Higgs Doublet Model ?, Phys. Rev. D 85, (2012) 095016 [arXiv:1112.3961 [hep-ph]].

[111] P. M. Ferreira, R. Santos, M. Sher and J. P. Silva, Implications of the LHC two-photon signal for two-Higgs-doublet models, Phys. Rev. D 85, (2012) 077703 [arXiv:1112.3277 [hep-ph]]; A. Arhrib, R. Benbrik and N. Gaur, $H \rightarrow \gamma \gamma$ in Inert Higgs Doublet Model, Phys. Rev. D 85, (2012) 095021 [arXiv:1201.2644 [hep-ph]].

[112] D. Carmi, A. Falkowski, E. Kuflik and T. Volansky, Interpreting LHC Higgs Results from Natural New Physics Perspective, JHEP 1207, (2012) 136 [arXiv:1202.3144 [hep-ph]]; A. Azatov, R. Contino and J. Galloway, Model-Independent Bounds on a Light Higgs, JHEP 1204, (2012) 127; J. Ellis and T. You, Global Analysis of Experimental Constraints on a Possible Higgs-Like Particle with Mass 125 GeV, JHEP 1206, (2012) 140 [arXiv:1204.0464 [hep-ph]].

[113] E. Gabrielli, B. Mele and M. Raidal, Has a Fermiophobic Higgs Boson been Detected at the LHC?, Phys. Lett. B 716, (2012) 322 [arXiv:1202.1796 [hep-ph]]; P. P. Giardino, K. Kannike, M. Raidal and A. Strumia, Reconstructing Higgs boson properties from the LHC and Tevatron data, JHEP 1206, 117 (2012) [arXiv:1203.4254 [hep-ph]].

[114] C. S. Li and T. C. Yuan, Qcd Correction To Charged Higgs Decay Of The Top Quark, Phys. Rev. D 42, 3088 (1990) [Erratum-ibid. D 47, 2156 (1993)]; A. Czarnecki and S. Davidson, 
QCD corrections to the charged Higgs decay of a heavy quark, Phys. Rev. D 48, 4183 (1993) [hep-ph/9301237]. 